\title{
External Review of the Quota Formulas - Statistical Appendix (Part A)
}




\title{
REPORT TO THE IMF EXECUTIVE BOARD OF THE QUOTA FORMULA REVIEW GROUP
}

\author{
STATISTICAL APPENDIX - PART A
}

May 1, 2000

Contents

Page

I. Existing and Alternative Data Sets for Use in Quota Formulas ................................. 3

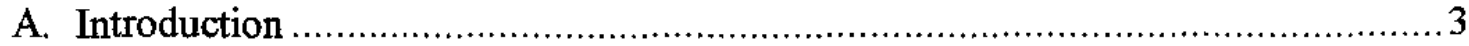

B. Eleventh Review of Quotas .................................................................... 3

C. Measures of Output and Population ............................................................ 3

D. Measures of Reserves and Current Account Data .......................................... 4

E. Variability Measures, Capital Flows, and Debt ..............................................

F. Qualitative and Dummy Variables …………..................................................

II. Estimation Results of Statistical Quota Formulas................................................55

A. List of Regression Equations...........................................................................5 56

III. Chow and Wald Tests for the Stability of Coefficient Estimates ...............................71

A. Chow Tests .................................................................................................72

B. Wald Tests: Tests of Structural Change with Unequal Variances...................... 74

IV. Nested Formulas with Vulnerability and Strength Variables .....................................77

A. Davidson-MacKinnon J Test.....................................................................78

B. Nested Models With Vulnerability and Strength Variables................................81

V. Hypothetical Quota Calculations for Past Quota Reviews According to the

Relative Size of the Equiproportional and Selective Elements. 83

Tables

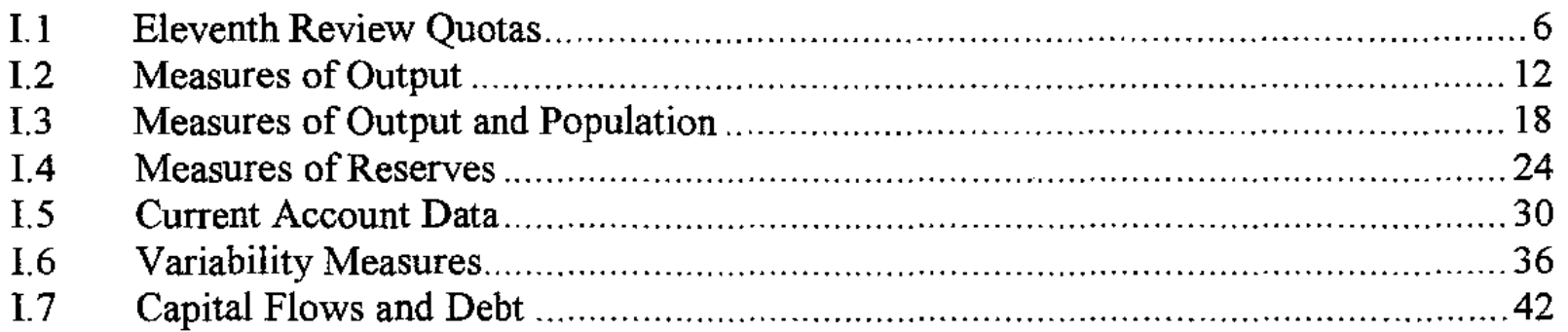

CInternational Monetary Fund. Not for Redistribution 


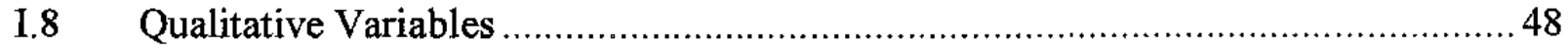

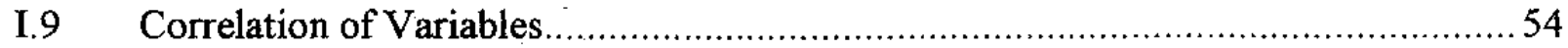

II. 1 Summary Statistics on Equations Fitted to Actual Quotas ................................59

II.2 Relative Contribution of Variables to Calculated Quotas ....................................6. 63

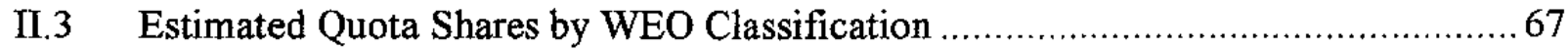

III.1.1 Chow Tests (Rolling)

III.1.2 Chow Tests (Cumulative) …................................................................. 73

III.2.1 Tests for Equality of Variances of Error Terms (F-test) _...................................... 76

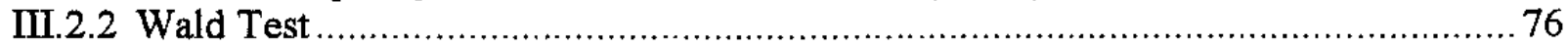

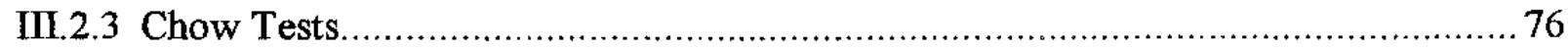

IV. Comparison of Coefficients from Nested Models and Ordinary Least Squares to Estimate the Relative Weights of Strength and Vulnerability Variables.

V.1 Summary of Simulated Quota Shares from Sixth Through Eleventh Reviews ......... 84 


\section{Section I. Existing and Alternative Data Sets for USE In Quota Formulas}

\section{A. Introduction}

1. This statistical appendix presents the data used in the estimation and analysis of alternative quota formulas suggested by the QFRG. Annual data up to 1994 have been compiled for 183 countries who participated in the Eleventh Quota Review to form the statistical database used in the estimation of alternative quota formulas. The IMF International Financial Statistics, and Balance of Payments databases comprise the major sources of data, supplemented by the IMF World Economic Outlook and IMF staff estimates. All data have been converted, where applicable, into millions of SDRs. A general description of the data, organized by major economic concepts, is presented below. This is followed by statistical data tables showing data for individual countries, sorted by actual (proposed) Eleventh Review quotas in descending order, along with a correlation matrix of certain variables.

\section{B. Eleventh Review of Quotas}

2. The dependent variable (Q) used in all quota formula estimations is the proposed or actual quota from the Eleventh Quota Review. Please refer to Financial Organization and Operations of the IMF, Pamphlet Series No. 45, Fifth Edition, pages 24-27, for an overview of the methodology used in the determination of quotas. The then-existing quota (QL) would be, in most cases, the proposed or actual quota from the Ninth Review. The calculated quota (CQ) is derived as the higher of the results of: (1) the reduced Bretton Woods formula and (2) the average of the lowest two results of four other formulas containing the same variables but with larger weights for external trade and variability of exports.

\section{Measures of Output and Population}

3. Five different measures of GDP/GNP were considered in the estimation of quota formulas. The "standard" GDP measure used in the Eleventh Quota Review is 1994 GDP (Y) measured at market exchange rates. Alternative national output measures include:

- Five-year (1990-94) average of GDP (YAVG),

- GDP valued at purchasing power parities (PPP) in a recent year (1994),

- Five-year (1990-94) average of GDP using a centered, five-year moving average of annual exchange rates as a conversion factor (YM5X), and

- GNP converted using the World Bank Atlas Method (YATL). 
4. The major source for GDP and exchange rate data is the IMF International Financial Statistics database. The data source for PPP-based GDP is the IMF World Economic Outlook database, and the World Bank's Global Development Indicators database is the source for 1994 GNP converted using the World Bank Atlas Method. In cases where there is a lack of reliable alternative measures of GDP, the 1994 GDP (Y) measure is usually substituted. Population (POP) is a country's 1994 population measured in millions of persons.

\section{Measures of Reserves and Current Account Data}

5. The standard reserves variable (R) used in the Eleventh Quota Review is a twelvemonth average of gold (valued at SDR 35 per fine ounce) and foreign exchange reserves, including SDR holdings, reserve positions in the Fund, and ECUs for 1994. An alternative measure used is reserves with gold measured at market prices (RESM). This is computed as a twelve-month average of gold (valued at market prices in 1994, with monthly prices ranging from SDR 261.02 per fine ounce to SDR 273.82 per fine ounce) and foreign exchange reserves, including holdings, reserve position in the Fund and ECUs for 1994.

6. Current Receipts (C) is the 1990-94 average of the sum of goods (exports f.o.b.), services (credit), income (credit), and private current transfers (credit) divided by the average SDR value for the same years. Current Payments (P) is the 1990-94 average of the sum of goods (imports, f.o.b.), services (debit), income (debit), and private current transfers (debit); divided by the average SDR value for the same years. Trade (TRADE) is measured as the average of current receipts and current payments.

\section{E. Variability Measures, Capital Flows, and Debt}

7. Several measures of variability were considered in the estimation of alternative quota formulas. The standard variability measure in the Eleventh Review uses the variability of current receipts (VC) which is defined as one standard deviation of current receipts from its five-year moving average centered on the third year, for the period 1982-94. Another measure captures the variability of both current receipts and capital and financial account credits (VCK), also defined as one standard deviation from its five-year moving average centered on the third year, for the period 1982-94. The variability of real effective exchange rates (VREC) (Source: IMF Information Notice System) is defined in terms of the deviation of the real effective exchange rate from a normal level, represented by a five-year moving average centered on the middle year.

8. Normal net capital flow is proxied by a four-year average of net private capital flows and includes errors and omissions for the period 1991-94.

9. Total external debt (DEBT) is defined as debt owed to non-residents repayable in foreign currency, goods, and services. Short-term external debt (STDEBT) for most developing countries was obtained from the BIS-IMF-OECD-World Bank (BIOW) database. 
The World Bank's Global Development Finance (GDF) database was used for developing countries for which data were not available from the BIOW. For the industrial countries, the Bank for International. Settlements database was used.

\section{F. Qualitative and Dummy Variables}

10. A capital market accessibility (KMACC) classification was used to derive an Openness Index. This classification (KMACC) ranks each country's ability to access capital . markets on a 1 to 4 scale, with a 1 given to countries with the easiest access, and a 4 given to those with the least access. The Openness Index (OPEN) has the reverse scale (computed as $1+(5-\mathrm{KMACC}))$.

11. Some dummy variables were not used directly as explanatory variables but were created in order to select sub-samples for estimation. NOTBW is a 0-1 dummy variable, which equals 1 if a country's calculated quota is based on a variant of the Bretton Woods Formula. Similarly, MEM20 equals 1 if the member joined the Fund in the past twenty years. Also, DDEV is a 0-1 dummy variable, which equals 1 if a country is classified as either a developing country or a transitional economy.

12. According to the WEO country classifications, advanced economies include the industrial countries of North America and Europe, Japan, and two newly industrialized Asian economies (Korea and Singapore). The countries in transition include the 15 members that were formerly part of the Soviet Union, the successor countries to the former Yugoslavia and Czechoslovakia, Albania, Hungary, Poland, Romania, and Mongolia. The rest of the members are classified as developing countries. Furthermore, San Marino, Palau, and the Marshall Islands are not currently classified by the WEO. We have classified San Marino as an industrial country, and Palau and Marshall Islands as developing countries.

13. The next section presents statistical data tables for the 183 countries that participated in the Eleventh Review of Quotas, organized along the same economic concepts presented above. 
Table I.1. Eleventh Review Quotas

\begin{tabular}{|c|c|c|c|c|c|c|}
\hline \multirow{3}{*}{ Regression Variable Name } & \multicolumn{2}{|c|}{$\begin{array}{l}\text { Actual } \\
\text { (Proposed) } \\
\text { Quotas }\end{array}$} & \multicolumn{2}{|c|}{$\begin{array}{l}\text { Then- } \\
\text { Existing } \\
\text { Quotas }\end{array}$} & \multicolumn{2}{|c|}{$\begin{array}{l}\text { Calculated Quotas } \\
\text { Based on Existing } \\
\text { 5-Formula System }\end{array}$} \\
\hline & $\begin{array}{l}\text { In millions of } \\
\quad \text { SDRs }\end{array}$ & $\begin{array}{l}\text { In percent } \\
\text { of total }\end{array}$ & $\begin{array}{l}\text { In millions of } \\
\text { SDRs }\end{array}$ & $\begin{array}{c}\text { In percent } \\
\text { of total }\end{array}$ & $\begin{array}{l}\text { In millions of } \\
\text { SDRs }\end{array}$ & $\begin{array}{c}\text { In percent } \\
\text { of total }\end{array}$ \\
\hline & \multicolumn{2}{|l|}{ Q } & \multicolumn{2}{|c|}{$\mathrm{QL}$} & \multicolumn{2}{|c|}{$\mathrm{QC}$} \\
\hline United States & $37,149.3$ & 17.521 & $26,526.8$ & 18.141 & $94,099.0$ & 17.251 \\
\hline Japan & $13,312.8$ & 6.279 & $8,241.5$ & 5.636 & $55,658.4$ & 10.204 \\
\hline Germany & $13,008.2$ & 6.135 & $8,241.5$ & 5.636 & $49,132.1$ & 9.007 \\
\hline France & $10,738.5$ & 5.065 & $7,414.6$ & 5.071 & $30,435.0$ & 5.580 \\
\hline United Kingdom & $10,738.5$ & 5.065 & $7,414.6$ & 5.071 & $27,227.4$ & 4.992 \\
\hline Italy & $7,055.5$ & 3.328 & $4,590.7$ & 3.139 & $22,804.1$ & 4.181 \\
\hline Saudi Arabia & $6,985.5$ & 3.295 & $5,130.6$ & 3.509 & $7,159.1$ & 1.312 \\
\hline Canada & $6,369.2$ & 3.004 & $4,320.3$ & 2.955 & $17,830.8$ & 3.269 \\
\hline Russia & $5,945.4$ & 2.804 & $4,313.1$ & 2.950 & $10,052.3$ & 1.843 \\
\hline Netherlands & $5,162.4$ & 2.435 & $3,444.2$ & 2.355 & $15,442.1$ & 2.831 \\
\hline China & $4,687.2$ & 2.211 & $3,385.2$ & 2.315 & $9,042.6$ & 1.658 \\
\hline Belgium & $4,605.2$ & 2.172 & $3,102.3$ & 2.122 & $13,248.5$ & 2.429 \\
\hline India & $4,158.2$ & 1.961 & $3,055.5$ & 2.090 & $4,156.0$ & 0.762 \\
\hline Switzerland & $3,458.5$ & 1.631 & $2,470.4$ & 1.689 & $8,697.2$ & 1.594 \\
\hline Australia & $3,236.4$ & 1.526 & $2,333.2$ & 1.596 & $6,554.6$ & 1.202 \\
\hline Spain & $3,048.9$ & 1.438 & $1,935.4$ & 1.324 & $11,343.3$ & 2.080 \\
\hline Brazil & $3,036.1$ & 1.432 & $2,170.8$ & 1.485 & $7,479.9$ & 1.371 \\
\hline Venezuela & $2,659.1$ & 1.254 & $1,951.3$ & 1.334 & $2,853.6$ & 0.523 \\
\hline Mexico & $2,585.8$ & 1.220 & $1,753.3$ & 1.199 & $7,246.7$ & 1.329 \\
\hline Sweden & $2,395.5$ & 1.130 & $1,614.0$ & 1.104 & $6,886.0$ & 1.262 \\
\hline Argentina & $2,117.1$ & 0.998 & $1,537.1$ & 1.051 & $3,487.7$ & 0.639 \\
\hline Indonesia & $2,079.3$ & 0.981 & $1,497.6$ & 1.024 & $4,318.0$ & 0.792 \\
\hline Austria & $1,872.3$ & 0.883 & $1,188.3$ & 0.813 & $6,972.5$ & 1.278 \\
\hline South Africa & $1,868.5$ & 0.881 & $1,365.4$ & 0.934 & $2,429.2$ & 0.445 \\
\hline Nigeria & $1,753.2$ & 0.827 & $1,281.6$ & 0.876 & $2,246.8$ & 0.412 \\
\hline Norway & $1,671.7$ & 0.788 & $1,104.6$ & 0.755 & $5,209.6$ & 0.955 \\
\hline Denmark & $1,642.8$ & 0.775 & $1,069.9$ & 0.732 & $5,450.0$ & 0.999 \\
\hline Korea & $1,633.6$ & 0.770 & 799.6 & 0.547 & $9,049.7$ & 1.659 \\
\hline Iran & $1,497.2$ & 0.706 & $1,078.5$ & 0.738 & $3,095.9$ & 0.568 \\
\hline Malaysia & $1,486.6$ & 0.701 & 832.7 & 0.569 & $5,608.4$ & 1.028 \\
\hline Kuwait & $1,381.1$ & 0.651 & 995.2 & 0.681 & $2,832.9$ & 0.519 \\
\hline Ukraine & $1,372.0$ & 0.647 & 997.3 & 0.682 & $2,172.1$ & 0.398 \\
\hline Poland & $1,369.0$ & 0.646 & 988.5 & 0.676 & $2,657.1$ & 0.487 \\
\hline Finland & $1,263.8$ & 0.596 & 861.8 & 0.589 & $3,463.0$ & 0.635 \\
\hline Algeria & $1,254.7$ & 0.592 & 914.4 & 0.625 & $1,811.0$ & 0.332 \\
\hline
\end{tabular}


Table I.1. Eleventh Review Quotas

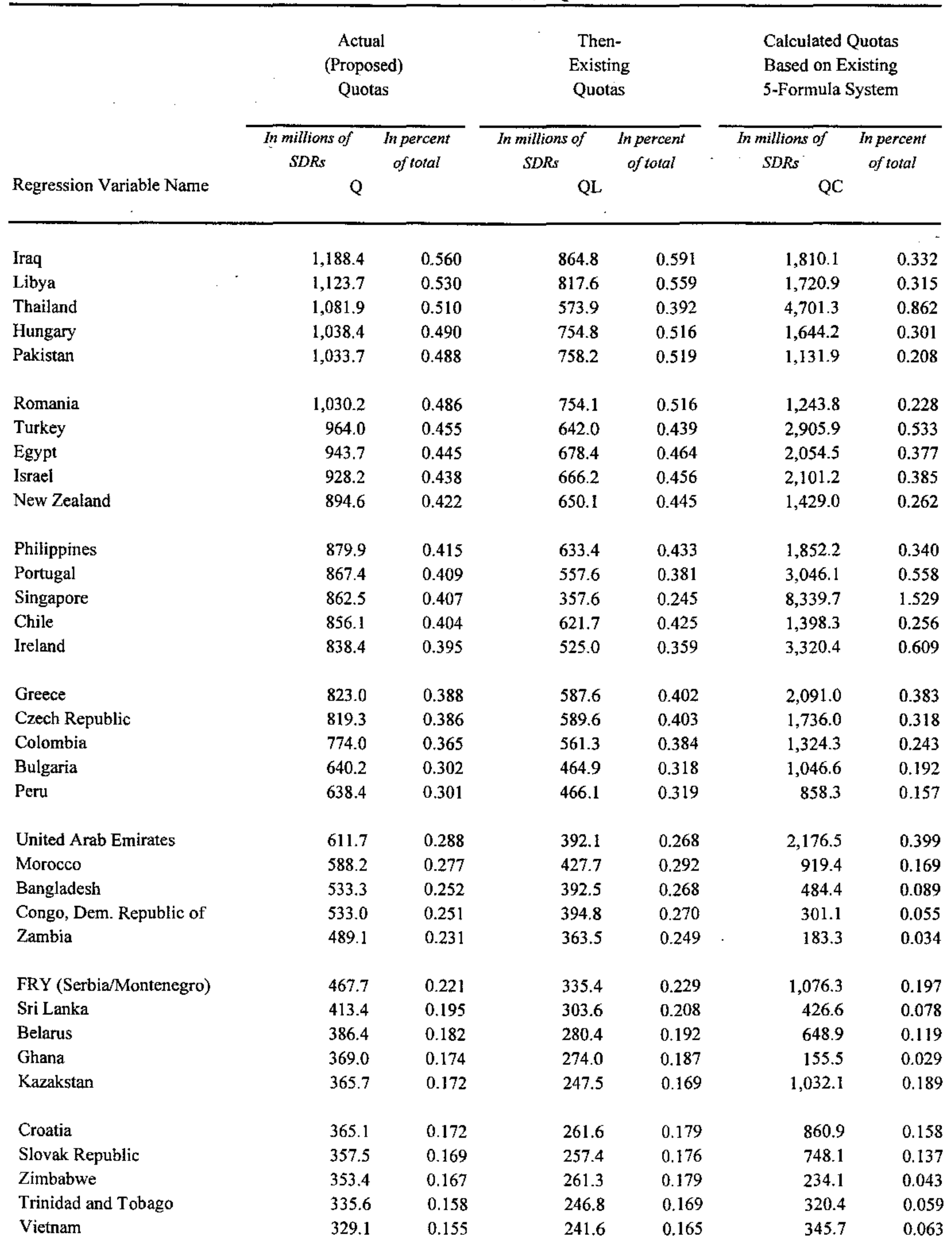


Table I.1. Eleventh Review Quotas

\begin{tabular}{|c|c|c|c|c|c|c|}
\hline \multirow[b]{3}{*}{ Regression Variable Name } & \multicolumn{2}{|c|}{$\begin{array}{c}\text { Actual } \\
\text { (Proposed) } \\
\text { Quotas }\end{array}$} & \multicolumn{2}{|c|}{$\begin{array}{l}\text { Then- } \\
\text { Existing } \\
\text { Quotas }\end{array}$} & \multicolumn{2}{|c|}{$\begin{array}{l}\text { Calculated Quotas } \\
\text { Based on Existing } \\
\text { 5-Formula System }\end{array}$} \\
\hline & $\begin{array}{c}\text { In millions of } \\
\text { SDRs }\end{array}$ & $\begin{array}{l}\text { In percent } \\
\text { of total }\end{array}$ & $\begin{array}{l}\text { In millions of } \\
\text { SDRs }\end{array}$ & $\begin{array}{l}\text { In percent } \\
\text { of total }\end{array}$ & $\begin{array}{l}\text { In millions of } \\
\text { SDRs }\end{array}$ & $\begin{array}{l}\text { In percent } \\
\text { of total }\end{array}$ \\
\hline & \multicolumn{2}{|l|}{ Q } & \multicolumn{2}{|l|}{ QL } & \multicolumn{2}{|l|}{ QC } \\
\hline Cote d'Ivoire & 325.2 & 0.153 & 238.2 & 0.163 & 379.0 & 0.069 \\
\hline Sudan & 315.1 & 0.149 & 233.1 & 0.159 & 198.5 & 0.036 \\
\hline Uruguay & 306.5 & 0.145 & 225.3 & 0.154 & 302.7 & 0.055 \\
\hline Ecuador & 302.3 & 0.143 & 219.2 & 0.150 & 517.2 & 0.095 \\
\hline Syrian Arab Republic & 293.6 & 0.138 & 209.9 & 0.144 & 723.7 & 0.133 \\
\hline Tunisia & 286.5 & 0.135 & 206.0 & 0.141 & 621.4 & 0.114 \\
\hline Angola & 286.3 & 0.135 & 207.3 & 0.142 & 516.1 & 0.095 \\
\hline Luxembourg & 279.1 & 0.132 & 135.5 & 0.093 & $1,587.8$ & 0.291 \\
\hline Uzbekistan & 275.6 & 0.130 & 199.5 & 0.136 & 495.4 & 0.091 \\
\hline Jamaica & 273.5 & 0.129 & 200.9 & 0.137 & 280.5 & 0.051 \\
\hline Kenya & 271.4 & 0.128 & 199.4 & 0.136 & 273.7 & 0.050 \\
\hline Qatar & 263.8 & 0.124 & 190.5 & 0.130 & 509.2 & 0.093 \\
\hline Myanmar & 258.4 & 0.122 & 184.9 & 0.126 & 628.4 & 0.115 \\
\hline Yemen, Republic of & 243.5 & 0.115 & 176.5 & 0.121 & 421.6 & 0.077 \\
\hline Slovenia & 231.7 & 0.109 & 150.5 & 0.103 & 776.9 & 0.142 \\
\hline Dominican Republic & 218.9 & 0.103 & 158.8 & 0.109 & 370.0 & 0.068 \\
\hline Brunei Darussalam & 215.2 & 0.101 & 150.0 & 0.103 & 553.7 & 0.102 \\
\hline Guatemala & 210.2 & 0.099 & 153.8 & 0.105 & 259.9 & 0.048 \\
\hline Panama & 206.6 & 0.097 & 149.6 & 0.102 & 372.0 & 0.068 \\
\hline Lebanon & 203.0 & 0.096 & 146.0 & 0.100 & 436.0 & 0.080 \\
\hline Tanzania & 198.9 & 0.094 & 146.9 & 0.100 & 142.8 & 0.026 \\
\hline Oman & 194.0 & 0.091 & 119.4 & 0.082 & 833.6 & 0.153 \\
\hline Cameroon & 185.7 & 0.088 & 135.1 & 0.092 & 285.3 & 0.052 \\
\hline Uganda & 180.5 & 0.085 & 133.9 & 0.092 & 86.5 & 0.016 \\
\hline Bolivia & 171.5 & 0.081 & 126.2 & 0.086 & 155.4 & 0.028 \\
\hline El Salvador & 171.3 & 0.081 & 125.6 & 0.086 & 192.5 & 0.035 \\
\hline Jordan & 170.5 & 0.080 & 121.7 & 0.083 & 434.1 & 0.080 \\
\hline Bosnia-Herzegovina & 169.1 & 0.080 & 121.2 & 0.083 & 392.5 & 0.072 \\
\hline Costa Rica & 164.1 & 0.077 & 119.0 & 0.081 & 282.4 & 0.052 \\
\hline Afghanistan, Islamic State of & 161.9 & 0.076 & 120.4 & 0.082 & 54.2 & 0.010 \\
\hline Senegal & 161.8 & 0.076 & 118.9 & 0.081 & 158.8 & 0.029 \\
\hline Azerbaijan & 160.9 & 0.076 & 117.0 & 0.080 & 251.2 & 0.046 \\
\hline Gabon & 154.3 & 0.073 & 110.3 & 0.075 & 383.3 & 0.070 \\
\hline Georgia & 150.3 & 0.071 & 111.0 & 0.076 & 108.4 & 0.020 \\
\hline Lithuania & 144.2 & 0.068 & 103.5 & 0.071 & 326.3 & 0.060 \\
\hline
\end{tabular}


Table 1.1. Eleventh Review Quotas

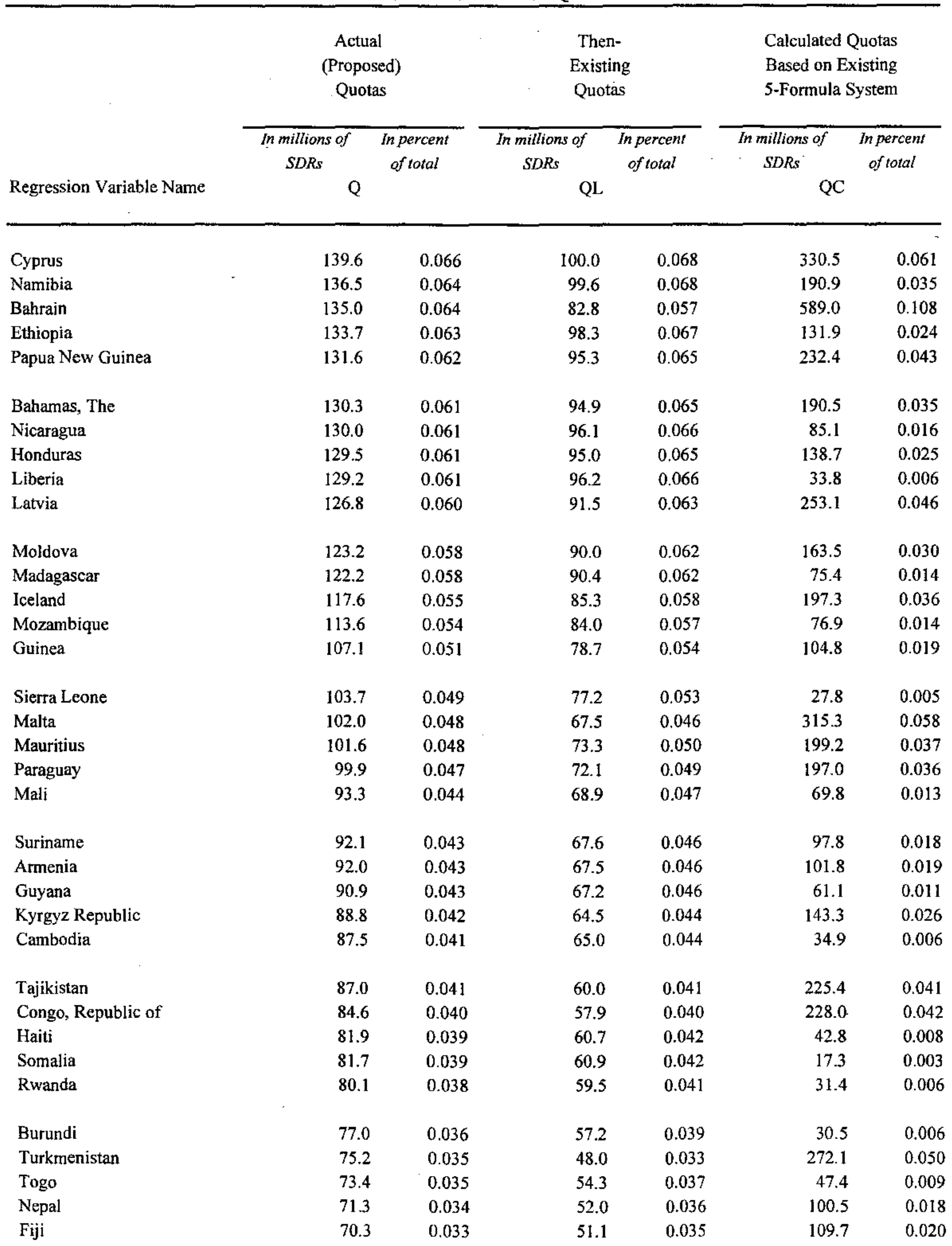


Table I.1. Eleventh Review Quotas

\begin{tabular}{|c|c|c|c|c|c|c|}
\hline \multirow[b]{3}{*}{ Regression Variable Name } & \multicolumn{2}{|c|}{$\begin{array}{l}\text { Actual } \\
\text { (Proposed) } \\
\text { Quotas }\end{array}$} & \multicolumn{2}{|c|}{$\begin{array}{l}\text { Then- } \\
\text { Existing } \\
\text { Quotas }\end{array}$} & \multicolumn{2}{|c|}{$\begin{array}{l}\text { Calculated Quotas } \\
\text { Based on Existing } \\
\text { 5-Formula System }\end{array}$} \\
\hline & $\begin{array}{l}\text { In millions of } \\
\text { SDRs }\end{array}$ & $\begin{array}{c}\text { In percent } \\
\text { of total }\end{array}$ & $\begin{array}{l}\text { In millions of } \\
\text { SDRs }\end{array}$ & $\begin{array}{c}\text { In percent } \\
\text { of total }\end{array}$ & $\begin{array}{c}\text { In millions of } \\
\text { SDRs }\end{array}$ & $\begin{array}{l}\text { In percent } \\
\text { of total }\end{array}$ \\
\hline & \multicolumn{2}{|l|}{$\mathrm{Q}$} & \multicolumn{2}{|c|}{$\mathrm{QL}$} & \multicolumn{2}{|c|}{$\mathrm{QC}$} \\
\hline Malawi & 69.4 & 0.033 & 50.9 & 0.035 & 74.3 & 0.014 \\
\hline Macedonia, FYR & 68.9 & 0.032 & 49.6 & 0.034 & 144.4 & 0.026 \\
\hline Barbados & 67.5 & 0.032 & 48.9 & 0.033 & 120.1 & 0.022 \\
\hline Niger & 65.8 & 0.031 & 48.3 & 0.033 & 67.3 & 0.012 \\
\hline Estonia & 65.2 & 0.031 & 46.5 & 0.032 & 167.1 & 0.031 \\
\hline Mauritania & 64.4 & 0.030 & 47.5 & 0.032 & 52.9 & 0.010 \\
\hline Botswana & 63.0 & 0.030 & 36.6 & 0.025 & 358.4 & 0.066 \\
\hline Benin & 61.9 & 0.029 & 45.3 & 0.031 & 73.3 & 0.013 \\
\hline Burkina Faso & 60.2 & 0.028 & 44.2 & 0.030 & 64.3 & 0.012 \\
\hline Chad & 56.0 & 0.026 & 41.3 & 0.028 & 42.2 & 0.008 \\
\hline Central African Republic & 55.7 & 0.026 & 41.2 & 0.028 & 35.6 & 0.007 \\
\hline Lao, People's Dem. Republic & 52.9 & 0.025 & 39.1 & 0.027 & 34.8 & 0.006 \\
\hline Mongolia & 51.1 & 0.024 & 37.1 & 0.025 & 85.8 & 0.016 \\
\hline Swaziland & 50.7 & 0.024 & 36.5 & 0.025 & 108.7 & 0.020 \\
\hline Albania & 48.7 & 0.023 & 35.3 & 0.024 & 86.4 & 0.016 \\
\hline Lesotho & 34.9 & 0.016 & 23.9 & 0.016 & 93.8 & 0.017 \\
\hline Equatorial Guinea & 32.6 & 0.015 & 24.3 & 0.017 & 8.2 & 0.001 \\
\hline Gambia, The & 31.1 & 0.015 & 22.9 & 0.016 & 29.2 & 0.005 \\
\hline Belize & 18.8 & 0.009 & 13.5 & 0.009 & 39.9 & 0.007 \\
\hline San Marino & 17.0 & 0.008 & 10.0 & 0.007 & 90.8 & 0.017 \\
\hline Vanuatu & 17.0 & 0.008 & 12.5 & 0.009 & 16.2 & 0.003 \\
\hline Djibouti & 15.9 & 0.007 & 11.5 & 0.008 & 29.1 & 0.005 \\
\hline Eritrea & 15.9 & 0.007 & 11.5 & 0.008 & 30.6 & 0.006 \\
\hline St. Lucia & 15.3 & 0.007 & 11.0 & 0.008 & 33.3 & 0.006 \\
\hline Guinea-Bissau & 14.2 & 0.007 & 10.5 & 0.007 & 9.9 & 0.002 \\
\hline Antigua and Barbuda & 13.5 & 0.006 & 8.5 & 0.006 & 51.9 & 0.010 \\
\hline Grenada & 11.7 & 0.006 & 8.5 & 0.006 & 16.6 & 0.003 \\
\hline Samoa & 11.6 & 0.005 & 8.5 & 0.006 & 11.6 & 0.002 \\
\hline Solomon Islands & 10.4 & 0.005 & 7.5 & 0.005 & 19.9 & 0.004 \\
\hline Cape Verde & 9.6 & 0.005 & 7.0 & 0.005 & 15.5 & 0.003 \\
\hline Comoros & 8.9 & 0.004 & 6.5 & 0.004 & 10.4 & 0.002 \\
\hline St. Kitts and Nevis & 8.9 & 0.004 & 6.5 & 0.004 & 12.8 & 0.002 \\
\hline Seychelles & 8.8 & 0.004 & 6.0 & 0.004 & 24.6 & 0.005 \\
\hline St. Vincent and the Grenadines & 8.3 & 0.004 & 6.0 & 0.004 & 16.2 & 0.003 \\
\hline Dominica & 8.2 & 0.004 & 6.0 & 0.004 & 11.2 & 0.002 \\
\hline
\end{tabular}


Table 1.1. Eleventh Review Quotas

\begin{tabular}{|c|c|c|c|c|c|c|}
\hline \multirow[b]{3}{*}{ Regression Variable Name } & \multicolumn{2}{|c|}{$\begin{array}{l}\text { Actual } \\
\text { (Proposed) } \\
\text { Quotas }\end{array}$} & \multicolumn{2}{|c|}{$\begin{array}{l}\text { Then- } \\
\text { Existing } \\
\text { Quotas }\end{array}$} & \multicolumn{2}{|c|}{$\begin{array}{l}\text { Calculated Quotas } \\
\text { Based on Existing } \\
\text { 5-Formula System }\end{array}$} \\
\hline & $\begin{array}{l}\text { In millions of } \\
\text { SDDRs }\end{array}$ & $\begin{array}{l}\text { In percent } \\
\text { of total }\end{array}$ & $\begin{array}{l}\text { In millions of } \\
\text { SDRs }\end{array}$ & $\begin{array}{l}\text { In percent } \\
\text { of total }\end{array}$ & $\begin{array}{l}\text { In millions of } \\
\text { SDRs }\end{array}$ & $\begin{array}{l}\text { In percent } \\
\text { of total }\end{array}$ \\
\hline & \multicolumn{2}{|l|}{$Q$} & \multicolumn{2}{|c|}{$\mathrm{QL}$} & \multicolumn{2}{|c|}{$\mathrm{QC}$} \\
\hline Maldives & 8.2 & 0.004 & 5.5 & 0.004 & 23.9 & 0.004 \\
\hline Sao Tome and Principe & 7.4 & 0.003 & 5.5 & 0.004 & 3.2 & 0.001 \\
\hline Tonga & 6.9 & 0.003 & 5.0 & 0.003 & 10.0 & 0.002 \\
\hline Bhutan & 6.3 & 0.003 & 4.5 & 0.003 & 14.4 & 0.003 \\
\hline Kiribati & 5.6 & 0.003 & 4.0 & 0.003 & 13.6 & 0.002 \\
\hline Micronesia, Fed. States of & 5.1 & 0.002 & 3.5 & 0.002 & 13.1 & 0.002 \\
\hline Marshall Islands & 3.5 & 0.002 & 2.5 & 0.002 & 7.8 & 0.001 \\
\hline Palau, Republic of & 3.1 & 0.001 & 2.3 & 0.002 & 5.7 & 0.001 \\
\hline Total & $212,029.0$ & 100.0 & $146,226.1$ & 100.0 & $545,459.1$ & 100.0 \\
\hline By WEO Classification & & & & & & \\
\hline Advanced Economies & $133,990.9$ & 63.2 & $90,248.6$ & 61.7 & $411,706.7$ & 75.5 \\
\hline Major Industrial Countries & $98,372.0$ & 46.4 & $66,750.0$ & 45.6 & $297,186.9$ & 54.5 \\
\hline Other Advanced Economies & $35,618.9$ & 16.8 & $23,498.6$ & 16.1 & $114,519.8$ & 21.0 \\
\hline Developing Countries & $61,922.5$ & 29.2 & $44,340.2$ & 30.3 & $104,840.4$ & 19.2 \\
\hline Net Creditors & $10,775.0$ & 5.1 & $7,795.4$ & 5.3 & $15,785.9$ & 2.9 \\
\hline $\begin{array}{l}\text { Net Debtors } \\
\text { of which HIPC or least }\end{array}$ & $51,147.5$ & 24.1 & $36,544.8$ & 25.0 & $89,054.5$ & 16.3 \\
\hline developed countries & $7,567.4$ & 3.6 & $5,555.7$ & 3.8 & $7,210.5$ & 1.3 \\
\hline Transition Economies & $16,115.6$ & 7.6 & $11,637.3$ & 8.0 & $28,912.1$ & 5.3 \\
\hline Total & $212,029.0$ & 100.0 & $146,226.1$ & 100.0 & $545,459.1$ & 100.0 \\
\hline
\end{tabular}


Table I.2. Measures of Output

\begin{tabular}{|c|c|c|c|c|c|c|}
\hline \multirow[b]{3}{*}{ Regression Variable Name } & \multirow{2}{*}{\multicolumn{2}{|c|}{$\begin{array}{c}\text { GDP at Market-- } \\
\text { Exchange Rates } \\
(1994)\end{array}$}} & \multicolumn{2}{|c|}{$\begin{array}{c}\begin{array}{c}\text { PPP-based GDP } \\
(1994)\end{array} \\
\end{array}$} & \multicolumn{2}{|c|}{$\begin{array}{c}\text { Five-year Average } \\
\text { of GDP } \\
(1990-94) \\
\end{array}$} \\
\hline & & & $\begin{array}{l}\text { In millions of } n \\
\text { SDRs }\end{array}$ & $\begin{array}{l}n \text { percent } \\
\text { of total }\end{array}$ & $\begin{array}{l}\text { In millions of } 1 \\
\text { SDRs }\end{array}$ & $\begin{array}{l}\text { In percent } \\
\text { of total }\end{array}$ \\
\hline & $Y$ & & PPP & & YAVG & \\
\hline United States & $4,844,380.8$ & 27.089 & $4,649,857.6$ & 21.261 & $4,508,151.1$ & 25.778 \\
\hline Japan & $3,275,179.3$ & 18.314 & $1,807,018.3$ & 8.262 & $2,730,703.6$ & 15.614 \\
\hline Germany & $1,429,097.5$ & 7.991 & $1,067,722.7$ & 4.882 & $1,311,822.4$ & 7.501 \\
\hline France & $929,655.0$ & 5.198 & $803,080.6$ & 3.672 & $904,473.6$ & 5.172 \\
\hline United Kingdom & $715,191.1$ & 3.999 & $754,269.4$ & 3.449 & $720,529.1$ & 4.120 \\
\hline Italy & $709,828.2$ & 3.969 & $740,475.3$ & 3.386 & $785,670.8$ & 4.493 \\
\hline Saudi Arabia & $83,933.0$ & 0.469 & 142.901 .3 & 0.653 & $83,941.9$ & 0.480 \\
\hline Canada & $382,194,1$ & 2.137 & $424,877.1$ & 1.943 & $411,966.6$ & 2.356 \\
\hline Russia & $194,803.3$ & 1.089 & $482,218.4$ & 2.205 & $333,739.6$ & 1.908 \\
\hline Netherlands & $233,497.0$ & 1.306 & $202,492.2$ & 0.926 & $221,257.9$ & 1.265 \\
\hline China & $377,831.1$ & 2.113 & $2,159,738.0$ & 9.875 & $343,617.0$ & 1.965 \\
\hline Belgium & $160,295.5$ & 0.896 & $132,381.2$ & 0.605 & $153,850.8$ & 0.880 \\
\hline India & $204,306.9$ & 1.142 & $876,514.5$ & 4.008 & $210,827.9$ & 1.206 \\
\hline Switzerland & $182,431.6$ & 1.020 & $113,113.5$ & 0.517 & $172,676.3$ & 0.987 \\
\hline Australia & $226,698.5$ & 1.268 & $239,522.8$ & 1.095 & $223,896.1$ & 1.280 \\
\hline Spain & $337,346.1$ & 1.886 & $372,321.4$ & 1.702 & $367,960.6$ & 2.104 \\
\hline Brazil & $394,324.3$ & 2.205 & $673,390.6$ & 3.079 & $322,697.2$ & 1.845 \\
\hline Venezuela & $40,600.9$ & 0.227 & $127,938.5$ & 0.585 & $40,320.8$ & 0.231 \\
\hline Mexico & $293,897.8$ & 1.643 & $535,174.9$ & 2.447 & $252,858.4$ & 1.446 \\
\hline Sweden & $138,599.3$ & 0.775 & $112,2 ! 2.3$ & 0.513 & $158,341.8$ & 0.905 \\
\hline Argentina & $196,916.0$ & 1.101 & $170,886.0$ & 0.781 & $150,996.8$ & 0.863 \\
\hline Indonesia & $123,553,8$ & 0.691 & $502,201.2$ & 2.296 & $102,701.5$ & 0.587 \\
\hline Austria & $138,382.5$ & 0.774 & $108,813.7$ & 0.498 & $127,999.5$ & 0.732 \\
\hline South Africa & $84,921.0$ & 0.475 & $115,277,4$ & 0.527 & $82,872.9$ & 0.474 \\
\hline Nigeria & $29,034.2$ & 0.162 & $99,719.1$ & 0.456 & $28,846.0$ & 0.165 \\
\hline Norway & $85,860.4$ & 0.480 & $61,958.4$ & 0.283 & $85,972.4$ & 0.492 \\
\hline Denmark & $101,646.3$ & 0.568 & $71,549.7$ & 0.327 & $101,241.7$ & 0.579 \\
\hline Korea & $265,993.0$ & 1.487 & $333,588.6$ & 1.525 & $224,995.7$ & 1.287 \\
\hline Iran & $74,231.6$ & 0.415 & $214,075.2$ & 0.979 & $352,644.3$ & 2.016 \\
\hline Malaysia & $50,648.5$ & 0.283 & $129,865.9$ & 0.594 & $40,944.0$ & 0.234 \\
\hline Kuwait & $17,363.5$ & 0.097 & $31,968.3$ & 0.146 & $14,058.5$ & 0.080 \\
\hline Ukraine & $25,672.1$ & 0.144 & $117,279.8$ & 0.536 & $133,060.6$ & 0.761 \\
\hline Poland & $64,676.6$ & 0.362 & $170,381.6$ & 0.779 & $57,104.8$ & 0.327 \\
\hline Finland & $68,185.3$ & 0.381 & $58,517.9$ & 0.268 & 79.845 .3 & 0.457 \\
\hline Algeria & $29,314.7$ & 0.164 & $105,916.2$ & 0.484 & $35,610.9$ & 0.204 \\
\hline
\end{tabular}


Table 1.2. Measures of Output

\begin{tabular}{|c|c|c|c|c|c|c|}
\hline \multirow[b]{3}{*}{ Regression Variable Name } & \multirow{2}{*}{\multicolumn{2}{|c|}{$\begin{array}{c}\begin{array}{c}\text { GDP at Market"- } \\
\text { Exchange Rates } \\
(1994)\end{array} \\
\text { In millions of In percent }\end{array}$}} & \multicolumn{2}{|c|}{$\begin{array}{c}\begin{array}{c}\text { PPP-based GDP "/ } \\
(1994)\end{array} \\
\end{array}$} & \multicolumn{2}{|c|}{$\begin{array}{c}\text { Five-year Average } \\
\text { of GDP } \\
(1990-94)\end{array}$} \\
\hline & & $\begin{array}{l}\text { In percent } \\
\text { of total }\end{array}$ & $\begin{array}{c}\text { In millions of } n \\
\text { SDRs }\end{array}$ & $\begin{array}{l}\text { percent } \\
\text { of total }\end{array}$ & $\begin{array}{l}\text { In millions of In } \\
\text { SDRs }\end{array}$ & $\begin{array}{l}\text { n percent } \\
\text { of total }\end{array}$ \\
\hline & Y & & PPP & & YAVG & \\
\hline Iraq & $24,332.8$ & 0.136 & n.a. & D.a. & $122,874.0^{2 /}$ & 0.703 \\
\hline Libya & $26,773.5$ & 0.150 & $37,041.9$ & 0.169 & $24,200.4$ & 0.138 \\
\hline Thailand & $99,994.4$ & 0.559 & $294,834.1$ & 1.348 & $80,943.4$ & 0.463 \\
\hline Hungary & $28,990.8$ & 0.162 & $43,508.8$ & 0.199 & $26,376.1$ & 0.151 \\
\hline Pakistan & $35,938.8$ & 0.201 & $196,322.3$ & 0.898 & $33,020.3$ & 0.189 \\
\hline Romania & $21,002.8$ & 0.117 & $53,109.6$ & 0.243 & $20,612.0$ & 0.118 \\
\hline Turkey & $91,508.1$ & 0.512 & $219,598.4$ & 1.004 & $113,764,5$ & 0.651 \\
\hline Egypt & $36,108.6$ & 0.202 & $162,779.9$ & 0.744 & $34,208.5$ & 0.196 \\
\hline Israel & $51,771.9$ & 0.289 & $60,153.8$ & 0.275 & $45,601.2$ & 0.261 \\
\hline New Zealand & $35,565.0$ & 0.199 & $39,237.7$ & 0.179 & $31,562.3$ & 0.180 \\
\hline Philippines & $44,761.2$ & 0.250 & $126,656.4$ & 0.579 & $37,433.8$ & 0.214 \\
\hline Portugal & $61,013.2$ & 0.341 & $74,908.8$ & 0.343 & $59,364.1$ & 0.339 \\
\hline Singapore & $49,617.3$ & 0.277 & $43,113,6$ & 0.197 & $37,225.1$ & 0.213 \\
\hline Chile & $36,434.6$ & 0.204 & $92,353.0$ & 0.422 & $28,966.0$ & 0.166 \\
\hline Ireland & $36,399.2$ & 0.204 & $35,454.7$ & 0.162 & $34,771.0$ & 0.199 \\
\hline Greece & $68,394.6$ & 0.382 & $52,440.5$ & 0.285 & $65,235.1$ & 0.379 \\
\hline Czech Republic & $26,316.4$ & 0.147 & $60,559.8$ & 0.277 & $26,999.7$ & 0.154 \\
\hline Colombia & $47,937.0$ & 0.268 & $153,542.8$ & 0.702 & $35,106.3$ & $0.20 \mathrm{I}$ \\
\hline Butgaria & $6,781.0$ & 0.038 & $31,524.9$ & 0.144 & $8,300.3$ & 0.047 \\
\hline Perl & $35,083,3$ & 0.196 & $63,540.8$ & 0.291 & $30,083.4$ & 0.172 \\
\hline United Arab Emirates & $25,611.8$ & 0.143 & $32,671,0$ & 0.149 & $25,158.0$ & 0.144 \\
\hline Moroceo & $21,199.9$ & 0.119 & $68,222.8$ & 0.312 & $20,015.5$ & 0.114 \\
\hline Bangladesh & $17,897.2$ & 0.100 & $116,829.1$ & 0.534 & $16,794.5$ & 0.096 \\
\hline Congo, Dem. Republic of & $4,071.0$ & 0.023 & $8,376.7$ & 0.038 & $6,214.5$ & 0.036 \\
\hline Zambia & $2,212.4$ & 0.012 & $5,138.8$ & 0.023 & $2,451.5$ & 0.014 \\
\hline FRY (Serbia/Montenegro) & $10,458.9$ & 0.058 & n.a. & n,a. & $71,750.7$ & 0.410 \\
\hline Sri Lanka & $8,186.1$ & 0.046 & $40,729.0$ & 0.186 & $6,995.4$ & 0.040 \\
\hline Belanus & $3,393.7$ & 0.019 & $40,093.0$ & 0.183 & $19,481.5$ & 0.111 \\
\hline Ghana & $3,614.1$ & 0.020 & $23,947.8$ & 0.109 & $4,326,9$ & 0.025 \\
\hline Kazakstan & $8,323.2$ & 0.047 & $39,989.9$ & 0.183 & $9,928.8$ & 0.057 \\
\hline Croatia & $9,936.2$ & 0.056 & $17,499.5$ & 0.080 & $21,230.5$ & 0.121 \\
\hline Slovak Republic & $9,618.9$ & 0.054 & $23,547.2$ & 0.108 & $16,460.0$ & 0.094 \\
\hline Zimbabwe & $4,063.7$ & 0.023 & $12,177.2$ & 0.056 & $5,331.8$ & 0.030 \\
\hline Trinidad and Tobago & $3,368.2$ & 0.019 & $7,942.9$ & 0.036 & $3,642.1$ & 0.021 \\
\hline Vietnam & $10,848.3$ & 0.061 & $8,617.0$ & 0.039 & $7,479.8$ & 0.043 \\
\hline
\end{tabular}


Table I.2. Measures of Output

\begin{tabular}{|c|c|c|c|c|c|c|}
\hline \multirow[b]{3}{*}{ Regression Variable Name } & \multicolumn{2}{|c|}{$\begin{array}{c}\text { GDF at Market" } \\
\text { Exchange Rates } \\
\frac{(1994)}{\text { In millions of In percent }}\end{array}$} & \multicolumn{2}{|c|}{$\frac{\text { PPP-based GDP }}{(1994)}$} & \multicolumn{2}{|c|}{$\begin{array}{c}\text { Five-year Average } \\
\text { of GDP } \\
(1990-94) \\
\text { In millions of In eercent }\end{array}$} \\
\hline & $\begin{array}{c}\text { In millions of } \\
\text { SDRs }\end{array}$ & $\begin{array}{l}\text { In percent } \\
\text { of total }\end{array}$ & $\begin{array}{c}\text { In millions of } n \\
\text { SDRs }\end{array}$ & $\begin{array}{l}\text { percent } \\
\text { of total }\end{array}$ & $\begin{array}{l}\text { In millions of Ir } \\
\text { SDRs }\end{array}$ & $\begin{array}{l}\text { In percent } \\
\text { of total }\end{array}$ \\
\hline & Y & & PPP & & YAVG & \\
\hline Cote d'Ivoire & $5,203.5$ & 0.029 & $28,050.1$ & 0.128 & $7,270.2$ & 0.042 \\
\hline Sudan & $5,706.9$ & 0.032 & $28,050.1$ & 0.128 & $9,762.1$ & 0.056 \\
\hline Uruguay & $11,350,3$ & 0.063 & $18,958.6$ & 0.087 & $8,641.2$ & 0.049 \\
\hline Ecuador & $11,598.5$ & 0.065 & $37,717.0$ & 0.172 & $9,459.0$ & 0.054 \\
\hline Syrian Arab Republic & $31,258.3$ & 0.175 & $56,650.4$ & 0.259 & $23,860.7$ & 0.136 \\
\hline Tunisia & $10,914.6$ & 0.061 & $34,218.6$ & 0.156 & $10,193.9$ & 0.058 \\
\hline Angola & $2,998.2$ & 0.017 & $10,409.0$ & 0.048 & $8,050.5$ & 0.046 \\
\hline Luxembourg & $11,436.4$ & 0.064 & $9,467.8$ & 0.043 & $9,071.2$ & 0.052 \\
\hline Uzbekistan & $3,975.0$ & 0.022 & $37,434.7$ & 0.171 & $15,918.3$ & 0.091 \\
\hline Jamaica & $2,964.0$ & 0.017 & $6,406.7$ & 0.029 & $2,757.0$ & 0.016 \\
\hline Kenya & $4,993.6$ & 0.028 & $26,0: 6.4$ & 0.119 & $5,424.1$ & 0.031 \\
\hline Qatar & $5,150.8$ & 0.029 & $5,373.2$ & 0.025 & $5,232.3$ & 0.030 \\
\hline Myanrnar & $55,267.5$ & 0.309 & $21,028.5$ & 0.096 & $25,005.6$ & 0.143 \\
\hline Yemen, Republic of & $15,754.8$ & 0.088 & $17,851.6$ & 0.082 & $11,245.4$ & 0.064 \\
\hline Slovenia & $10,009.1$ & 0.056 & $16,157.8$ & 0.074 & $34,531.1$ & 0.197 \\
\hline Dominican Republic & $7,229.1$ & 0.040 & $20,205.8$ & 0.092 & $6,241.7$ & 0.036 \\
\hline Brunei Danussalam & $3,057.6$ & 0.017 & n.a. & n.a. & $2,857.1$ & 0.016 \\
\hline Guatemala & $9,068.4$ & 0.051 & $26,122.6$ & 0.119 & $7,431.9$ & 0.042 \\
\hline Panama & $5,401.9$ & 0.030 & $11,735.4$ & 0.054 & $4,699.6$ & 0.027 \\
\hline Lebanon & $6,362.9$ & 0.036 & $6,314.5$ & 0.029 & $4,208.6$ & 0.024 \\
\hline Tanzania & $3,150.7$ & 0.018 & $12,500.7$ & 0.057 & $3,245.8$ & 0.019 \\
\hline Oman & $9,023.4$ & 0.050 & $13,446.9$ & 0.061 & $8,573.3$ & 0.049 \\
\hline Cameroon & $4,308.3$ & 0.024 & $20,348.2$ & 0.093 & $7,668.6$ & 0.044 \\
\hline Uganda & $3,688.1$ & 0.021 & $15,880.2$ & 0.073 & $2,675.2$ & 0.015 \\
\hline Bolivia & $4,172.7$ & 0.023 & $12,019.3$ & 0.055 & $3,957.0$ & 0.023 \\
\hline El Salvador & $5,661.3$ & 0.032 & $10,650.4$ & 0.049 & $4,534.3$ & 0.026 \\
\hline Jordan $\frac{5}{\pi}$ & $4,199,5$ & 0.023 & $14,796.2$ & 0.068 & $3,564.4$ & 0.020 \\
\hline Bosnia-Herzegovina & $1,487.1$ & 0.008 & n.a. & n.a. & $1,487.1^{2 /}$ & 0.009 \\
\hline Costa Rica & $5,806.8$ & 0.032 & $14,230.8$ & 0.065 & $4,861.4$ & 0.028 \\
\hline Afghanistan, Islamic State of & $1,651.0$ & 0.009 & $11,754.3$ & 0.054 & $53,562.8$ & 0.306 \\
\hline Senegal & $2,702.0$ & 0.015 & $9,996.0$ & 0.046 & $3,816,9$ & 0.022 \\
\hline Azerbaijan & $1,287.4$ & 0.007 & $8,054.6$ & 0.037 & $6,382.6$ & 0.036 \\
\hline Gabon & $2,927,1$ & 0.016 & $2,803.8$ & 0.013 & $3,743.4$ & $0.02 \mathrm{I}$ \\
\hline Georgia & 870.4 & 0.005 & $5,763.4$ & 0.026 & $7,917.9$ & 0.045 \\
\hline Lithuania & $3,877,9$ & 0.022 & $7,365.8$ & 0.034 & $8,043.4$ & 0.046 \\
\hline
\end{tabular}


Table I.2. Measures of Output

\begin{tabular}{|c|c|c|c|c|c|c|}
\hline \multirow[b]{3}{*}{ Regression Variable Name } & \multicolumn{2}{|c|}{$\begin{array}{c}\text { GDP at Market } \\
\text { Exchange Rates } \\
\text { (1994) }\end{array}$} & \multicolumn{2}{|c|}{$\begin{array}{c}\text { PPP-based GDP } \\
(1994) \\
\end{array}$} & \multicolumn{2}{|c|}{$\begin{array}{l}\begin{array}{l}\text { Five-year Average } \\
\text { of GDP } \\
(1990-94) \\
\text { millions of In percent }\end{array}\end{array}$} \\
\hline & $\begin{array}{l}\text { M milions of } \\
\text { SDRs }\end{array}$ & $\begin{array}{l}\text { In percent } \\
\text { of total }\end{array}$ & $\begin{array}{c}\text { Inmillons of n } \\
\text { SDRs }\end{array}$ & of total & SDRs & $\begin{array}{l}\text { npercent } \\
\text { of total }\end{array}$ \\
\hline & $Y$ & & PPP & & YAVG & \\
\hline Cyprus & $5,178.2$ & 0.029 & $6,203.3$ & 0.028 & $4,623.6$ & 0.026 \\
\hline Namibia & $2,044.5$ & 0.011 & $2,049.0$ & 0.009 & $1,972.6$ & 0.011 \\
\hline Bahrain & $3,394.6$ & 0.019 & $5,335.8$ & 0.024 & $3,410.8$ & 0.020 \\
\hline Ethiopia & $3,624.0$ & 0.020 & $15,917.5$ & 0.073 & $5,095.5$ & 0.029 \\
\hline Papua New Guinea & $3,736.8$ & 0.021 & $6,958.2$ & 0.032 & $3,122.6$ & 0.018 \\
\hline Bahamas, The & $2,392.3$ & 0.013 & $2,945.2$ & 0.013 & $2,112.3$ & 0.012 \\
\hline Nicaragua & $1,293.0$ & 0.007 & $6,458.6$ & 0.030 & $2,690.5$ & 0.015 \\
\hline Honduras & $2,397.4$ & 0.013 & $8,088.6$ & 0.037 & $2,365.2$ & 0.014 \\
\hline Liberia & 528.3 & 0.003 & $1,626.0$ & 0.007 & $1,254.1$ & 0.007 \\
\hline Latvia & $2,547.7$ & 0.014 & $8,850.9$ & 0.040 & $6,031.5$ & 0.034 \\
\hline Moldova & 987.1 & 0.006 & $5,884,0$ & 0.027 & $5,862.0$ & 0.034 \\
\hline Madagascar & $2,079.3$ & 0.012 & $6,607.5$ & 0.030 & 2.170 .4 & 0.012 \\
\hline Iceland & $4,344.6$ & 0.024 & $3,480.9$ & 0.016 & $4,627.1$ & 0.026 \\
\hline Mozambique & $1,000.8$ & 0.006 & $8,172.8$ & 0.037 & $1,000.8^{3 /}$ & 0.006 \\
\hline Guinea & $2,371.2$ & 0.013 & $3,069.0$ & 0.014 & $2,296.8$ & 0.013 \\
\hline Sierra Leone & 647.2 & 0.004 & $1,998.3$ & 0.009 & 529.2 & 0.003 \\
\hline Malta & $1,883.5$ & 0.011 & $3,302.9$ & 0.015 & $1,827.6$ & 0.010 \\
\hline Mauritius & $2,447.0$ & 0.014 & $10,387.9$ & 0.047 & $2,205.7$ & 0.013 \\
\hline Paraguay & $5,466.4$ & 0.031 & $12,474.2$ & 0.057 & $4,682.8$ & 0.027 \\
\hline Mali & $1,212.5$ & 0.007 & $3,546.9$ & 0.016 & $1,710.5$ & 0.010 \\
\hline Suriname & 272.3 & 0.002 & $1,757.5$ & 0.008 & $1,905.9$ & 0.011 \\
\hline Armenia & 453.6 & 0.003 & $5,399.0$ & 0.025 & $453.6^{3 /}$ & 0.003 \\
\hline Guyana & 377.8 & 0.002 & $1,301.9$ & 0.006 & 305.4 & 0.002 \\
\hline Kyrgyz Republic & 778.3 & 0.004 & $6,116.7$ & 0.028 & $4,088.4$ & 0.023 \\
\hline Cambodia & $1,682.5$ & 0.009 & $2,849.4$ & 0.013 & $1,252.6$ & 0.007 \\
\hline Tajikistan, & 357.7 & 0.002 & $4,785.3$ & 0.022 & $2,498.0$ & 0.014 \\
\hline Congo, Retpublic of & $1,235.8$ & 0.007 & $4,617.6$ & 0.021 & $1,858.7$ & 0.011 \\
\hline Haiti & $1,286.1$ & 0.007 & $4,949.8$ & 0.023 & $1,677.4$ & 0.010 \\
\hline Somalia & 343.4 & 0.002 & $5,480.1$ & 0.025 & 826.1 & 0.005 \\
\hline Rwanda & 524.2 & 0.003 & $1,878.2$ & 0.009 & $1,335.5$ & 0.008 \\
\hline Burundi & 746.5 & 0.004 & $2,800.9$ & 0.013 & 783.6 & 0.004 \\
\hline Turkmenistan & $5,130.0$ & 0.029 & $6,751.7$ & 0.031 & $3,864.7$ & 0.022 \\
\hline Togo & 672.8 & 0.004 & $3,343.6$ & 0.015 & 999.6 & 0.006 \\
\hline Nepal & $2,816.9$ & 0.016 & $17,574.0$ & 0.080 & $2,557,2$ & 0.015 \\
\hline Fiji & $1,265.2$ & 0.007 & $3,298.6$ & 0.015 & $1,115.2$ & 0.006 \\
\hline
\end{tabular}


Table I.2. Measures of Output

\begin{tabular}{|c|c|c|c|c|c|c|}
\hline \multirow[b]{3}{*}{ Regression Variable Name } & $\begin{array}{r}\text { GDP at } M \\
\text { Exchange } \\
\text { (1994 }\end{array}$ & $\begin{array}{l}\text { Larket"- } \\
\text { Rates } \\
\text { 9) } \\
\text { forcent }\end{array}$ & \multicolumn{2}{|c|}{$\begin{array}{c}\begin{array}{c}\text { PPP-based GDP } \\
(1994)\end{array} \\
\end{array}$} & \multicolumn{2}{|c|}{$\begin{array}{c}\begin{array}{c}\text { Five-year Average } \\
\text { of GDP } \\
(1990-94)\end{array} \\
\text { millions of In percent }\end{array}$} \\
\hline & SDRS & of total & SDRs & $\begin{array}{l}\text { npercent } \\
\text { of total }\end{array}$ & $\begin{array}{l}\text { In millions of } \\
\text { SDRs }\end{array}$ & $\begin{array}{l}\text { In percent } \\
\text { of total }\end{array}$ \\
\hline & $Y$ & & PPP & & YAVG & \\
\hline Malawi & 896.2 & 0.005 & $4,753,5$ & 0.022 & $1,309.7$ & 0.007 \\
\hline Macedonia, FYR & $1,998.8$ & 0.011 & $3,300.3$ & 0.015 & $30,771.7$ & 0.176 \\
\hline Barbados & $1,207.9$ & 0.007 & $1,615.2$ & 0.007 & $1,206.6$ & 0.007 \\
\hline Niger & 1.091 .7 & 0.006 & $4,659.1$ & 0.021 & $1,565.3$ & 0.009 \\
\hline Estonia & $1,613.3$ & 0.009 & $5,705.6$ & 0.031 & $1,120.9$ & 0.006 \\
\hline Mauritania & 706.1 & 0.004 & $2,481.1$ & 0.011 & 748.6 & 0.004 \\
\hline Botswana & $2,891.8$ & 0.016 & $5,638.2$ & 0.025 & $2,739.9$ & 0.016 \\
\hline Benin & $1,063.8$ & 0.006 & $6,119.6$ & 0.028 & $1,362.8$ & 0.008 \\
\hline Burkina Faso & $1,295.0$ & 0.007 & $5,284.7$ & 0.024 & $1,901.5$ & 0.011 \\
\hline Chad & 579.8 & 0.003 & $3,126.6$ & 0.014 & 825.3 & 0.005 \\
\hline Central African Repubiic & 597.4 & 0.003 & $2,295.0$ & 0.010 & 916.3 & 0.005 \\
\hline Lao, People's Dem. Republic & $1,077.4$ & 0.006 & $7,248.6$ & 0.033 & 851.2 & 0.005 \\
\hline Mongolia & 479.4 & 0.003 & $1,846.8$ & 0.008 & 955.1 & 0.005 \\
\hline Swaziland & 741.7 & 0.004 & $2,583.7$ & 0.012 & 682.6 & 0.004 \\
\hline Albania & $1,376.2$ & 0.008 & $1,187.2$ & 0.005 & $1,030.3$ & 0.006 \\
\hline Lesotho & 543.7 & 0.003 & $2,36 \vdots .8$ & 0.011 & 531.4 & 0.003 \\
\hline Equatorial Guinea & 83.8 & 0.000 & 130.9 & 0.001 & 103.5 & 0.001 \\
\hline Gambia, The & 210.5 & 0.001 & $23,947.8$ & 0.109 & 216.6 & 0.001 \\
\hline Belize & 385.1 & 0.002 & 354.6 & 0.002 & 344.9 & 0.002 \\
\hline San Marino & 424.1 & 0.002 & n,a. & na. & 401.3 & 0.002 \\
\hline Vanuatu & 149.8 & 0.001 & 242.2 & 0.001 & 134.1 & 0.001 \\
\hline Djibouti & 340.4 & 0.002 & 432.0 & 0.002 & 330.5 & 0.002 \\
\hline Eritrea & 382.7 & 0.002 & n.a. & n.a. & 408.7 & 0.002 \\
\hline St. Lucia & 355.8 & 0.002 & 354.0 & 0.002 & 340.3 & 0.002 \\
\hline Guinea-Bissau & 164.6 & 0.001 & 538.0 & 0.002 & 724.2 & 0.004 \\
\hline Antigua and Barbuda & 347.9 & 0.002 & 333.6 & 0.002 & 313.1 & 0.002 \\
\hline Grenada & 181.7 & 0.001 & 293.5 & 0.001 & 176.0 & 0.001 \\
\hline Samoa & 95.9 & 0.001 & 225.5 & 0.001 & 115.1 & 0.001 \\
\hline Solomon Islands & 206.4 & 0.001 & 558.4 & 0.003 & 160.6 & 0.001 \\
\hline Cape Verde & 242.6 & 0.001 & 401.9 & 0.002 & 242.3 & 0.001 \\
\hline Comoros & 129.7 & 0.001 & 443.6 & 0.002 & 174.4 & 0.001 \\
\hline St. Kitts and Nevis & 155.8 & 0.001 & 139.5 & 0.001 & 132.7 & 0.001 \\
\hline Seychelles & 340.6 & 0.002 & 304.9 & 0.001 & 305.3 & 0.002 \\
\hline St. Vincent and the Grenadin & 166.9 & 0.001 & 164.4 & 0.001 & 161.0 & 0.001 \\
\hline Dominica & 151.2 & 0.001 & 138.3 & 0.001 & 136.9 & 0.001 \\
\hline
\end{tabular}


Table 1.2. Measures of Output

\begin{tabular}{|c|c|c|c|c|c|c|}
\hline \multirow[b]{3}{*}{ Regression Variable Name } & \multirow{2}{*}{\multicolumn{2}{|c|}{$\begin{array}{c}\text { GDP at Market -- } \\
\text { Exchange Rates } \\
(1994) \\
\text { In millions of In percent }\end{array}$}} & \multicolumn{2}{|c|}{$\begin{array}{c}\text { PPP-based GDP }{ }^{1 /} \\
\frac{(1994)}{\text { In millions of } n \text { percent }}\end{array}$} & \multicolumn{2}{|c|}{$\begin{array}{c}\text { Five-year Average } \\
\text { of GDP } \\
(1990-94) \\
\end{array}$} \\
\hline & & & $\begin{array}{l}\text { Inmillions of } n \\
\text { SDRs }\end{array}$ & $\begin{array}{l}\text { n percent } \\
\text { of total }\end{array}$ & $\begin{array}{l}\text { In millions of In } \\
\text { SDRs }\end{array}$ & $\begin{array}{l}\text { In percent } \\
\text { of total }\end{array}$ \\
\hline & \multicolumn{2}{|l|}{ Y } & \multicolumn{2}{|l|}{ PPP } & \multicolumn{2}{|l|}{ YAVG } \\
\hline Maldives & 168.2 & 0.001 & 156.7 & 0.001 & 135.3 & 0.001 \\
\hline Sao Tome and Principe & 34.6 & 0.000 & 44.0 & 0.000 & 37.0 & 0.000 \\
\hline Tonga & 113.6 & 0.001 & 96.9 & 0.000 & 104.5 & 0.001 \\
\hline Bhutan & 181.5 & 0.001 & 395.7 & 0.002 & 183.8 & 0.001 \\
\hline Kiribati & 28.1 & 0.000 & 26.6 & 0.000 & 24.8 & 0.000 \\
\hline Micronesia, Fed. States of & 143.5 & 0.001 & n.a. & n.a. & 127.4 & 0.001 \\
\hline Marshall Islands & 65.0 & 0.000 & n.a. & n.a. & $67.8^{4 \prime}$ & 0.000 \\
\hline Palau, Republic of & 62.1 & 0.000 & z.a. & n.a. & 46.7 & 0.000 \\
\hline Total & $17,883,525$ & 100 & $21,870,655$ & 100 & $17,488,284$ & 100 \\
\hline \multicolumn{7}{|l|}{ By WEO Classification } \\
\hline Advanced Economies & $14,543,427.8$ & 81.3 & $12,382,030.6$ & 56.6 & $13,580,213.7$ & 77.7 \\
\hline Major Inđustrial Countries & $12,285,526.1$ & 68.7 & $10,247,301.0$ & 46.9 & $11,373,317,1$ & 65.0 \\
\hline Other Advanced Economies & $2,257,901.6$ & 12.6 & $2,134,729.6$ & 9.8 & $2,206,896.6$ & 12.6 \\
\hline Developing Countries & $2,892,895.0$ & 16.2 & $8,283,308.0$ & 37.9 & $3,032,069.3$ & 17.3 \\
\hline Net Creditors & $170,913.7$ & 1.0 & $263,402.6$ & 1.2 & $164,021.3$ & 0.9 \\
\hline $\begin{array}{l}\text { Net Debtors } \\
\text { of which HIPC or least }\end{array}$ & $2,721,981.2$ & 15.2 & $8,019,905.3$ & 36.7 & $2,868,048.0$ & 16.4 \\
\hline developing countries & $185,859.0$ & 1.0 & $548,606.5$ & 2.5 & $224,994.4$ & 1.3 \\
\hline Transition Economies & $447,202.8$ & 2.5 & $1,205,316.3$ & 5.5 & $876,001.1$ & 5.0 \\
\hline Total & $17,883,526$ & 100 & $21,870,655$ & 100.0 & $17,488,284$ & 100 \\
\hline
\end{tabular}

1/ Source: IMF World Economic Outlook data base.

2/ Average of 1990-93 GDP.

3/ 1994 GDP.

4/ 1995 data. 


\begin{tabular}{|c|c|c|c|c|c|c|}
\hline \multirow[b]{3}{*}{ Regression Variable Name } & \multicolumn{2}{|c|}{$\begin{array}{c}\text { Five-year Average of } \\
\text { GDP (using centered, } \\
\text { five-year moving averages } \\
\text { of annual exchange rates } \\
\text { as conversion factors) } \\
(1990-94)\end{array}$} & \multicolumn{2}{|c|}{$\begin{array}{c}\text { GNP } \\
\text { Converted } \\
\text { using the } \\
\text { World Bank } \\
\text { Atlas Method }{ }^{1 /} \\
\text { (1994) }\end{array}$} & \multicolumn{2}{|c|}{$\begin{array}{l}\text { Population } \\
\text { (1994) }\end{array}$} \\
\hline & $\begin{array}{l}\text { In millions of } \\
\text { SDRs }\end{array}$ & $\begin{array}{l}\text { In percent } \\
\text { of total }\end{array}$ & $\begin{array}{l}\text { In millions of } \\
\text { SDRs }\end{array}$ & $\begin{array}{l}\text { In percent } \\
\text { of total }\end{array}$ & $\begin{array}{l}\text { In millions of } \\
\text { persons }\end{array}$ & $\begin{array}{l}\text { In percent } \\
\text { of total }\end{array}$ \\
\hline & \multicolumn{2}{|c|}{ YM5X } & \multicolumn{2}{|l|}{ YATL } & \multicolumn{2}{|c|}{ POP } \\
\hline United States & $4,874,255.0$ & 26.658 & $4,772,390.9$ & 26.683 & 257.6 & 4.689 \\
\hline Japan & $3,042,381.4$ & 16.639 & $3,084,996.2$ & 17.249 & 124.5 & 2.267 \\
\hline Germany & $1,469,926.0$ & 8.039 & $1,449,192.6$ & 8.103 & 81.2 & 1.478 \\
\hline France & $964,103.6$ & 5.273 & $948,156.7$ & 5.301 & 57.7 & 1.050 \\
\hline United Kingdom & $765,511.9$ & 4.187 & $748,537.4$ & 4.185 & 57.9 & 1.054 \\
\hline Italy & $779,541.2$ & 4.263 & $764,268.4$ & 4.273 & 57.1 & 1.039 \\
\hline Saudi Arabia & $88,742.8$ & 0.485 & $90,337.4$ & 0.505 & 17.1 & 0.312 \\
\hline Canada & $410,479.7$ & 2.245 & $401,220.4$ & 2.243 & 28.8 & 0.523 \\
\hline Russia & $194,803.3^{2 /}$ & 1.065 & $239,491.2$ & 1.339 & 148.7 & 2.707 \\
\hline Netherlands & $240,895.9$ & 1.317 & $240,946.8$ & 1.347 & 15.3 & 0.279 \\
\hline China & $454,418.6$ & 2.485 & $449,923.4$ & 2.516 & $1,196.4$ & 21.779 \\
\hline Belgium & $167,039.8$ & 0.914 & $165,258.1$ & 0.924 & 10.1 & 0.183 \\
\hline India & $241,022.8$ & 1.318 & $187,802.8$ & 1.050 & 901.5 & 16.410 \\
\hline Switzerland & $185,000.2$ & 1.012 & $185,613.7$ & 1.038 & 6.9 & 0.126 \\
\hline Australia & $240,425.0$ & 1.315 & $226,859.3$ & 1.268 & 17.7 & 0.321 \\
\hline Spain & $\therefore 374,177.0$ & 2.046 & $365,407.3$ & 2.043 & 39.1 & 0.712 \\
\hline Brazil & $394,324.3^{2 t}$ & 2.157 & $335,508.8$ & 1.876 & 159.2 & 2.898 \\
\hline Venezuela & $53,724.7$ & 0.294 & $41,089.8$ & 0.230 & 20.7 & 0.377 \\
\hline Mexico & $263,262.0$ & 1.440 & $287,195.9$ & 1.606 & 91.2 & 1.660 \\
\hline Sweden & $155,749.6$ & 0.852 & $146,320.7$ & 0.818 & 8.8 & 0.159 \\
\hline Argentina & $185,772.1$ & 1.016 & $194,728.9$ & 1.089 & 33.8 & 0.615 \\
\hline Indonesia & $121,092.3$ & 0.662 & $117,864.1$ & 0.659 & 189.1 & 3.443 \\
\hline Austria & $140,514.1$ & 0.768 & $138,618.9$ & 0.775 & 8.0 & 0.145 \\
\hline South Africa & $79,111.1$ & 0.433 & $87,175.9$ & 0.487 & 39.7 & 0.722 \\
\hline Nigeria & $54,054.3$ & 0.296 & $16,575.4$ & 0.093 & 105.3 & 1.916 \\
\hline Norway & $92,815.2$ & 0.508 & $90,309.6$ & 0.505 & 4.3 & 0.078 \\
\hline Denmark & $110,181.3$ & 0.603 & $106,866.5$ & 0.598 & 5.2 & 0.094 \\
\hline Korea & $282,571.7$ & 1.545 & $262,494.5$ & 1.468 & 44.1 & 0.802 \\
\hline Iran & $74,231.6^{2 \prime}$ & 0.406 & $74,231.6^{2 /}$ & 0.415 & 64.2 & 1.168 \\
\hline Malaysia & $51,664.0$ & 0.283 & $48,487.1$ & 0.271 & 19.3 & 0.350 \\
\hline Kuwait & $17,716.3$ & 0.097 & $21,569.2$ & 0.121 & 1.4 & 0.026 \\
\hline Ukraine & $25,672.1^{2 /}$ & 0.140 & $47,583.7$ & 0.266 & 52.2 & 0.951 \\
\hline Poland & $79,325.8$ & 0.434 & $65,417.2$ & 0.366 & 38.5 & 0.700 \\
\hline Finland & $77,819.5$ & 0.426 & $67,829.2$ & 0.379 & 5.1 & 0.092 \\
\hline Algeria & $35,913.7$ & 0.196 & $31,926.3$ & 0.179 & 26.7 & 0.486 \\
\hline
\end{tabular}


Table L.3. Measures of Output and Population

\begin{tabular}{|c|c|c|c|c|c|c|}
\hline \multirow[b]{3}{*}{ Regression Variable Name } & \multicolumn{2}{|c|}{$\begin{array}{c}\text { Five-year Average of } \\
\text { GDP (using centered, } \\
\text { five-year moving averages } \\
\text { of annual exchange rates } \\
\text { as conversion factors) } \\
(1990-94)\end{array}$} & \multicolumn{2}{|c|}{$\begin{array}{c}\text { GNP } \\
\text { Converted } \\
\text { using the } \\
\text { World Bank } \\
\text { Atlas Method }{ }^{1 /} \\
(1994) \\
\end{array}$} & \multicolumn{2}{|c|}{$\begin{array}{c}\text { Population } \\
\text { (1994) }\end{array}$} \\
\hline & $\begin{array}{l}\text { In millions of } \\
\quad \text { SDRs }\end{array}$ & $\begin{array}{l}\text { ercent } \\
\text { total }\end{array}$ & $\begin{array}{l}\text { In millions of } \\
\text { SDRs }\end{array}$ & $\begin{array}{l}\text { In percent } \\
\text { of toral }\end{array}$ & $\begin{array}{l}\text { In millions of } \\
\text { persons }\end{array}$ & $\begin{array}{l}\text { In percent } \\
\text { of total }\end{array}$ \\
\hline & \multicolumn{2}{|c|}{ YM5X } & \multicolumn{2}{|l|}{ YATL } & \multicolumn{2}{|c|}{ POP } \\
\hline Iraq & $24,332.8^{2 t}$ & 0.133 & $24,332.8^{2 t}$ & 0.136 & 19.5 & 0.354 \\
\hline Libya & $27,308.5$ & 0.149 & $26,773.5^{2 /}$ & 0.150 & 4.7 & 0.086 \\
\hline Thailand & $99,343.7$ & 0.543 & $97,585.1$ & 0.546 & 58.6 & 1.066 \\
\hline Hungary & $29,318.2$ & 0.160 & $27,830.6$ & 0.156 & 10.3 & 0.187 \\
\hline Pakistan & $37,419.4$ & 0.205 & $38,202.9$ & 0.214 & 122.8 & 2.235 \\
\hline Romania & $21,002.8^{2}$ & 0.115 & $19,689.3$ & 0.110 & 22.8 & 0.415 \\
\hline Turkey & $91,508.1^{2 /}$ & 0.500 & $110,359.9$ & 0.617 & 60.2 & 1.096 \\
\hline Egypt & $39,688.9$ & 0.217 & $34,397.4$ & 0.192 & 56.5 & 1.028 \\
\hline Israel & $55,054.4$ & 0.301 & $52,154.2$ & 0.292 & 5.3 & 0.096 \\
\hline New Zealand & $36,302.1$ & 0.199 & $32,214.9$ & 0.180 & 3.5 & 0.063 \\
\hline Philippines & $44,756.9$ & 0.245 & $43,853.2$ & 0.245 & 65.7 & 1.195 \\
\hline Portugal & $66,305.2$ & 0.363 & $64,469.0$ & 0.360 & 9.9 & 0.179 \\
\hline Singapore & $48,209.4$ & 0.264 & $47,645.0$ & 0.266 & 2.9 & 0.052 \\
\hline Chile & $38,301.6$ & 0.209 & $32,950.7$ & 0.184 & 13.8 & 0.251 \\
\hline Ireland & $41,163.5$ & 0.225 & $34,775.6$ & 0.194 & 3.6 & 0.065 \\
\hline Greece & $74,508.5$ & 0.407 & $71,454.7$ & 0.400 & 10.4 & 0.188 \\
\hline Czech Republic & $32,898.4$ & 0.180 & $23,826.1$ & 0.133 & 10.3 & 0.188 \\
\hline Colombia & $47,049.6$ & 0.257 & $42,730.2$ & 0.239 & 34.0 & 0.618 \\
\hline Bulgaria & $6,781.0^{2 f}$ & 0.037 & $7,357.8$ & 0.041 & 9.1 & 0.166 \\
\hline Peru & $35,083.3^{2 /}$ & 0.192 & $34,274.1$ & 0.192 & 22.5 & 0.409 \\
\hline United Arab Emirates & $27,029.6$ & 0.148 & $27,837.8$ & 0.156 & 2.1 & 0.038 \\
\hline Morocco & $21,601.5$ & 0.118 & $21,277.0$ & 0.119 & 26.1 & 0.475 \\
\hline Bangladesh & $18,697.1$ & 0.102 & $25,514.4$ & 0.143 & 115.2 & 2.097 \\
\hline Congo, Dem. Republic of & $4,071.0^{2 /}$ & 0.022 & $4,891.8$ & 0.027 & 41.2 & 0.751 \\
\hline Zambia & $2,212.4^{2 l}$ & 0.012 & $2,193.2$ & 0.012 & 8.9 & 0.163 \\
\hline FRY (Serbia/Montenegro) & $10,458.9^{2 t}$ & 0.057 & $10,458.9^{2 /}$ & 0.058 & 10.5 & 0.191 \\
\hline Sri Lanka & $8,245.7$ & 0.045 & $7,991.6$ & 0.045 & 17.6 & 0.321 \\
\hline Belarus & $3,393.7^{2 /}$ & 0.019 & $16,082.2$ & 0.090 & 10.3 & 0.188 \\
\hline Ghana & $4,573.7$ & 0.025 & $4,329.1$ & 0.024 & 16.5 & 0.299 \\
\hline Kazakstan & $8,323.2^{2 /}$ & 0.046 & $15,331.0$ & 0.086 & 16.9 & 0.308 \\
\hline Croatia & $9,936.2^{2 /}$ & 0.054 & $8,708.1$ & 0.049 & 4.8 & 0.088 \\
\hline Slovak Republic & $9,618.9^{2 /}$ & 0.053 & $9,125.2$ & 0.051 & 5.3 & 0.097 \\
\hline Zimbabwe & $5,400.8$ & 0.030 & $4,895.9$ & 0.027 & 10.7 & 0.196 \\
\hline Trinidad and Tobago & $3,710.8$ & 0.020 & $3,415.5$ & 0.019 & 1.3 & 0.023 \\
\hline Vietnam & $12,032.4$ & 0.066 & $9,811.7$ & 0.055 & 70.8 & 1.289 \\
\hline
\end{tabular}


Table L.3. Measures of Output and Population

\begin{tabular}{|c|c|c|c|c|c|c|}
\hline \multirow[b]{3}{*}{ Regression Variable Name } & \multicolumn{2}{|c|}{$\begin{array}{l}\text { Five-year Average of } \\
\text { GDP (using centered, } \\
\text { five-year moving averages } \\
\text { of annual exchange rates } \\
\text { as conversion factors) } \\
(1990-94)\end{array}$} & \multicolumn{2}{|c|}{$\begin{array}{c}\text { GNP } \\
\text { Converted } \\
\text { using the } \\
\text { World Bank } \\
\text { Atlas Method }{ }^{1 /} \\
(1994)\end{array}$} & \multicolumn{2}{|c|}{$\begin{array}{l}\text { Population } \\
\text { (1994) }\end{array}$} \\
\hline & $\begin{array}{l}\text { In millions of } \\
\text { SDRs }\end{array}$ & $\begin{array}{l}\text { In percent } \\
\text { oftotal }\end{array}$ & $\begin{array}{l}\text { In millions of } \\
\text { SDRs }\end{array}$ & $\begin{array}{l}\text { In percent } \\
\text { of total }\end{array}$ & $\begin{array}{l}\text { In millions of } \\
\text { persons }\end{array}$ & $\begin{array}{c}\text { In percent } \\
\text { of total }\end{array}$ \\
\hline & \multicolumn{2}{|c|}{ YM5X } & \multicolumn{2}{|l|}{ YATL } & \multicolumn{2}{|c|}{ POP } \\
\hline Cote dTvoire & $7,139.9$ & 0.039 & $6,193.7$ & 0.035 & 13.3 & 0.242 \\
\hline Sudan & $5,706.9^{2 t}$ & 0.031 & $5,007.4$ & 0.028 & 28.1 & 0.512 \\
\hline Uruguay & $12,597.6$ & 0.069 & $10,335.2$ & 0.058 & 3.2 & 0.057 \\
\hline Ecuador & $12,124.6$ & 0.066 & $10,004.5$ & 0.056 & 11.0 & 0.200 \\
\hline Syrian Arab Republic & $31,764.4$ & 0.174 & $10,101.5$ & 0.056 & 13.3 & 0.242 \\
\hline Tunisia & $11,521.6$ & 0.063 & $10,741.1$ & 0.060 & 8.6 & 0.156 \\
\hline Angola & $2,998.2^{2 t}$ & 0.016 & $1,640.2$ & 0.009 & 10.3 & 0.187 \\
\hline Luxembourg & $10,460.0$ & 0.057 & $11,083.3$ & 0.062 & 0.4 & 0.007 \\
\hline Uzbekistan & $3,975.0^{2 /}$ & 0.022 & $14,734.7$ & 0.082 & 21.5 & 0.392 \\
\hline Jamaica & $3,837.3$ & 0.021 & $2,548.4$ & 0.014 & 2.4 & 0.044 \\
\hline Kenya & $5,973.8$ & 0.033 & $4,735.0$ & 0.026 & 28.1 & 0.512 \\
\hline Qatar & $5,537.3$ & 0.030 & $5,251.3$ & 0.029 & 0.6 & 0.010 \\
\hline Myanmar & $57,653.4$ & 0.315 & $55,267.5^{2 /}$ & 0.309 & 44.6 & 0.812 \\
\hline Yemen, Republic of & $12,726.6$ & 0.070 & $3,039.0$ & 0.017 & 12.3 & 0.224 \\
\hline Slovenia & $10,009.1^{2 /}$ & 0.055 & $10,082.6$ & 0.056 & 2.0 & 0.036 \\
\hline Dominican Republic & $7,709.8$ & 0.042 & $6,988.0$ & 0.039 & 7.6 & 0.139 \\
\hline Brunei Darussalam & $3,180.2$ & 0.017 & $3,057.6^{2 \prime}$ & 0.017 & 0.3 & 0.005 \\
\hline Guatemala & $9,258.7$ & 0.051 & $8,612.5$ & 0.048 & 10.0 & 0.183 \\
\hline Panama & $5,296.4$ & 0.029 & $5,159.7$ & 0.029 & 2.6 & 0.047 \\
\hline Lebanon & $7,168.8$ & 0.039 & $5,826.2$ & 0.033 & 2.8 & 0.051 \\
\hline Tanzania & $3,835.4$ & 0.021 & $3,302.9$ & 0.018 & 28.0 & 0.510 \\
\hline Oman & $9,328.1$ & 0.051 & $7,234.2$ & 0.040 & 2.0 & 0.036 \\
\hline Cameroon & $6,498.1$ & 0.036 & $6,540.6$ & 0.037 & 12.5 & 0.228 \\
\hline Uganda & $3,640.6$ & 0.020 & $2,530.7$ & 0.014 & 19.9 & 0.363 \\
\hline Bolivia & $4,494.4$ & 0.025 & $4,206.8$ & 0.024 & 8.1 & 0.147 \\
\hline El Salvador & $5,809.9$ & 0.032 & $5,297.9$ & 0.030 & 5.5 & 0.100 \\
\hline Jordan & $4,876.1$ & 0.027 & $4,170.4$ & 0.023 & 4.9 & 0.090 \\
\hline Bosnia-Herzegovina & $1,487.1^{2 /}$ & 0,008 & $1,487.1^{2}$ & 0.008 & 4.5 & 0.081 \\
\hline Costa Rica & $5,829.6$ & 0.032 & $5,639.4$ & 0.032 & 3.2 & 0.058 \\
\hline Afghanistan, Islamic State of & $64,620.1$ & 0.353 & $1,651.0^{2 t}$ & 0.009 & 17.7 & 0.322 \\
\hline Senegal & $3,455.1$ & 0.019 & $2,704.7$ & 0.015 & 7.9 & 0.144 \\
\hline Azerbaijan & $1,287.4^{2}$ & 0.007 & $2,745.5$ & 0.015 & 7.3 & 0.133 \\
\hline Gabon & $3,716.1$ & 0.020 & $3,034.0$ & 0.017 & 1.1 & 0.019 \\
\hline Georgia & $870.4^{2 /}$ & 0.005 & $1,999.1$ & 0.011 & 5.4 & 0.098 \\
\hline Lithuania & $3,877.9^{2 t}$ & 0.021 & $4,615.6$ & 0.026 & 3.8 & 0.069 \\
\hline
\end{tabular}


Table I.3. Measures of Output and Population

\begin{tabular}{|c|c|c|c|c|c|c|}
\hline \multirow[b]{3}{*}{ Regression Variable Name } & \multicolumn{2}{|c|}{$\begin{array}{l}\text { Five-year Average of } \\
\text { GDP (using centered, } \\
\text { five-year moving averages } \\
\text { of annual exchange rates } \\
\text { as conversion factors) } \\
(1990-94)\end{array}$} & \multicolumn{2}{|c|}{$\begin{array}{c}\text { GNP } \\
\text { Converted } \\
\text { using the } \\
\text { World Bank } \\
\text { Atlas Method } \\
\text { (1994) }\end{array}$} & \multicolumn{2}{|c|}{$\begin{array}{c}\begin{array}{c}\text { Population } \\
(1994)\end{array} \\
\end{array}$} \\
\hline & $\begin{array}{l}\text { In millions of } \\
\quad \text { SDRs }\end{array}$ & torcent & $\begin{array}{l}\text { In millions of } \\
\text { SDRs }\end{array}$ & $\begin{array}{l}\text { In percent } \\
\text { of total }\end{array}$ & $\begin{array}{l}\text { In millions of } \\
\text { persons }\end{array}$ & $\begin{array}{l}\text { In percent } \\
\text { of total }\end{array}$ \\
\hline & \multicolumn{2}{|c|}{ YM5X } & \multicolumn{2}{|c|}{ YATL } & \multicolumn{2}{|c|}{ POP } \\
\hline Cyprus & $5,344.2$ & 0.029 & $5,204.4$ & 0.029 & 0.7 & 0.013 \\
\hline Namibia & $2,144.2$ & 0.012 & $2,230.1$ & 0.012 & 1.5 & 0.027 \\
\hline Bahrain & $3,860.5$ & 0.021 & $3,201.5$ & 0.018 & 0.5 & 0.010 \\
\hline Ethiopia & $4,685.7$ & 0.026 & $4,110.5$ & 0.023 & 56.9 & 1.036 \\
\hline Papua New Guinea & $3,470.2$ & 0.019 & $3,624.2$ & 0.020 & 3.9 & 0.071 \\
\hline Bahamas, The & $2,080.4$ & 0.011 & $2,242.7$ & 0.013 & 0.3 & 0.005 \\
\hline Nicaragua & $1,293.0^{2 t}$ & 0.007 & 960.7 & 0.005 & 4.3 & 0.078 \\
\hline Honduras & $2,691.5$ & 0.015 & $2,485.1$ & 0.014 & 5.6 & 0.102 \\
\hline Liberia & $1,487.4$ & 0.008 & $528.3^{2 /}$ & 0.003 & 2.6 & 0.048 \\
\hline Latvia & $2,547.7^{2}$ & 0.014 & $3,936.4$ & 0.022 & 2.7 & 0.049 \\
\hline Moldova & $987.1^{2 /}$ & 0.005 & $1,790.6$ & 0.010 & 4.4 & 0.080 \\
\hline Madagascar & $2,452.9$ & 0.013 & $2,150.8$ & 0.012 & 13.9 & 0.252 \\
\hline Iceland & $4,695.2$ & 0.026 & $4,594.9$ & 0.026 & 0.3 & 0.005 \\
\hline Mozambique & $1,000.8^{2 \prime}$ & 0.005 & $1,184.9$ & 0.007 & 17.2 & 0.312 \\
\hline Guinea & $2,782.7$ & 0.015 & $2,493.8$ & 0.014 & 6.3 & 0.115 \\
\hline Sierra Leone & 689.4 & 0.004 & 513.6 & 0.003 & 4.3 & 0.078 \\
\hline Malta & $2,031.4$ & 0.011 & $2,003.3$ & 0.011 & 0.4 & 0.007 \\
\hline Mauritius & $2,518.0$ & 0.014 & $2,474.9$ & 0.014 & 1.1 & 0.020 \\
\hline Paraguay & $5,637.0$ & 0.031 & $5,102.1$ & 0.029 & 4.6 & 0.084 \\
\hline Mali & $1,671.8$ & 0.009 & $1,673.5$ & 0.009 & 10.1 & 0.184 \\
\hline Suriname & $272.3^{2 t}$ & 0.001 & 226.8 & 0.001 & 0.4 & 0.007 \\
\hline Armenia & $453.6^{2 t}$ & 0.002 & $1,188.1$ & 0.007 & 3.7 & 0.067 \\
\hline Guyana & 411.5 & 0.002 & 300.3 & 0.002 & 0.8 & 0.015 \\
\hline Kyrgyz Republic & $778.3^{2 /}$ & 0.004 & $1,743.6$ & 0.010 & 4.5 & 0.083 \\
\hline Cambodia & $1,682.5^{2 t}$ & 0.009 & $1,653.8$ & 0.009 & 9.3 & 0.169 \\
\hline Tajikistan & $357.7^{2 /}$ & 0.002 & $1,521.9$ & 0.009 & 5.7 & 0.104 \\
\hline Congo, Republic of & $1,681.7$ & 0.009 & $1,268.4$ & 0.007 & 2.4 & 0.044 \\
\hline Haiti & $1,740.7$ & 0.010 & $1,293.8$ & 0.007 & 6.9 & 0.126 \\
\hline Somalia & $1,054.7$ & 0.006 & $343.4^{2 t}$ & 0.002 & 9.0 & 0.163 \\
\hline Rwanda & $1,114.3$ & 0.006 & 634.6 & 0.004 & 7.6 & 0.137 \\
\hline Burundi & 742.3 & 0.004 & 680.6 & 0.004 & 6.0 & 0.108 \\
\hline Turkmenistan & $5,130.0^{2 /}$ & 0.028 & $3,273.0$ & 0.018 & 3.9 & 0.071 \\
\hline Togo & 947.1 & 0.005 & 885.3 & 0.005 & 3.9 & 0.071 \\
\hline Nepal & $2,845.5$ & 0.016 & $2,912.3$ & 0.016 & 20.8 & 0.379 \\
\hline Fiji & $1,271.5$ & 0.007 & $1,228.5$ & 0.007 & 0.8 & 0.014 \\
\hline
\end{tabular}


Table 1.3. Measures of Output and Population

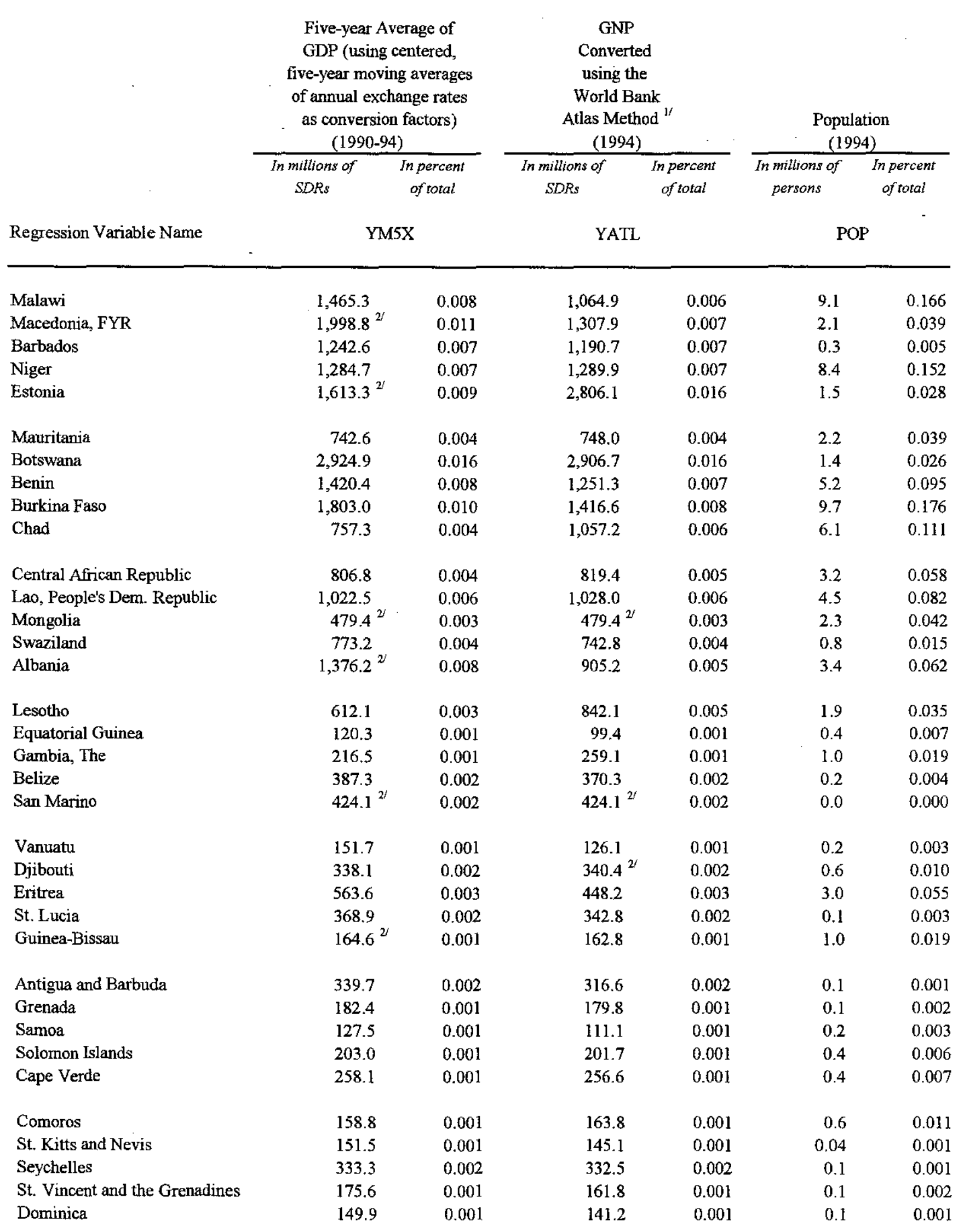


Table I.3. Measures of Output and Population

\begin{tabular}{|c|c|c|c|c|c|c|}
\hline \multirow[b]{3}{*}{ Regression Variable Name } & \multicolumn{2}{|c|}{$\begin{array}{c}\text { Five-year Average of } \\
\text { GDP (using centered, } \\
\text { five-year moving averages } \\
\text { of annual exchange rates } \\
\text { as conversion factors) } \\
(1990-94)\end{array}$} & \multicolumn{2}{|c|}{$\begin{array}{c}\text { GNP } \\
\text { Converted } \\
\text { using the } \\
\text { World Bank } \\
\text { Atlas Method }{ }^{1 /} \\
\text { (1994) }\end{array}$} & \multicolumn{2}{|c|}{$\begin{array}{c}\begin{array}{c}\text { Population } \\
(1994)\end{array} \\
\end{array}$} \\
\hline & $\begin{array}{c}\text { In millions of } \\
\text { SDRs }\end{array}$ & $\begin{array}{l}\text { In percent } \\
\text { of total }\end{array}$ & $\begin{array}{l}\text { In millions of } \\
\text { SDRs }\end{array}$ & $\begin{array}{l}\text { In percent } \\
\text { of total }\end{array}$ & $\begin{array}{l}\text { In millions of } \\
\text { persons }\end{array}$ & $\begin{array}{l}\text { In percent } \\
\text { of total }\end{array}$ \\
\hline & \multicolumn{2}{|c|}{ YM5X } & \multicolumn{2}{|l|}{ YATL } & \multicolumn{2}{|c|}{ POP } \\
\hline Maldives & 170.2 & 0.001 & 152.4 & 0.001 & 0.2 & 0.004 \\
\hline Sao Tome and Principe & $34.6^{2 /}$ & 0.000 & 31.3 & 0.000 & 0.1 & 0.002 \\
\hline Tonga & 97.5 & 0.001 & 112.1 & 0.001 & 0.1 & 0.002 \\
\hline Bhutan & 201.5 & 0.001 & 171.2 & 0.001 & 1.6 & 0.029 \\
\hline Kiribati & 28.8 & 0.000 & 46.9 & 0.000 & 0.1 & 0.001 \\
\hline Micronesia, Fed. States of & 138.7 & 0.001 & 141.2 & 0.001 & 0.5 & 0.008 \\
\hline Marshall Islands & 67.7 & 0.000 & $65.0^{2 t}$ & 0.000 & 0.1 & 0.001 \\
\hline Palau, Republic of & 57.8 & 0.000 & $62.1^{2 f}$ & 0.000 & $0.02^{3 /}$ & 0.000 \\
\hline Total & $18,284,637.3$ & 100.0 & $17,885,388.0$ & 100.0 & $5,493.4$ & 100.0 \\
\hline By WEO Classification & & & & & & \\
\hline Advanced Economies & $14,710,510.6$ & 80.5 & $14,484,103.2$ & 81.0 & 865.2 & 15.7 \\
\hline Major Industrial Countries & $12,306,198.9$ & 67.3 & $12,168,762.8$ & 68.0 & 664.7 & 12.1 \\
\hline Other Advanced Economies & $2,404,311.6$ & 13.1 & $2,315,340.5$ & 12.9 & 200.5 & 3.6 \\
\hline Developing Countries & $3,105,365.3$ & 17.0 & $2,855,767.2$ & 16.0 & $4,209.7$ & 76.6 \\
\hline Net Creditors & $178,842.9$ & 1.0 & $182,061.1$ & 1.0 & 28.2 & 0.5 \\
\hline $\begin{array}{l}\text { Net Debtors } \\
\text { of which HIPC or least }\end{array}$ & $2,926,522.4$ & 16.0 & $2,673,706.1$ & 14.9 & $4,181.5$ & 76.1 \\
\hline developed countries & $266,317.5$ & 1.5 & $184,180.5$ & 1.0 & 729.6 & 13.3 \\
\hline Transition Economies & $468,761.5$ & 2.6 & $\mathbf{5 4 5 , 5 1 7 . 9}$ & 3.1 & 418.5 & 7.6 \\
\hline Total & $18,284,637.4$ & 100.0 & $17,885,388.3$ & 100.0 & $5,493.4$ & 100.0 \\
\hline
\end{tabular}

1/ Source: 1999 World Bank Development Indicators data base.

2/ 1994 GDP.

3/ 1995 data. 
Table L4. Measures of Reserves

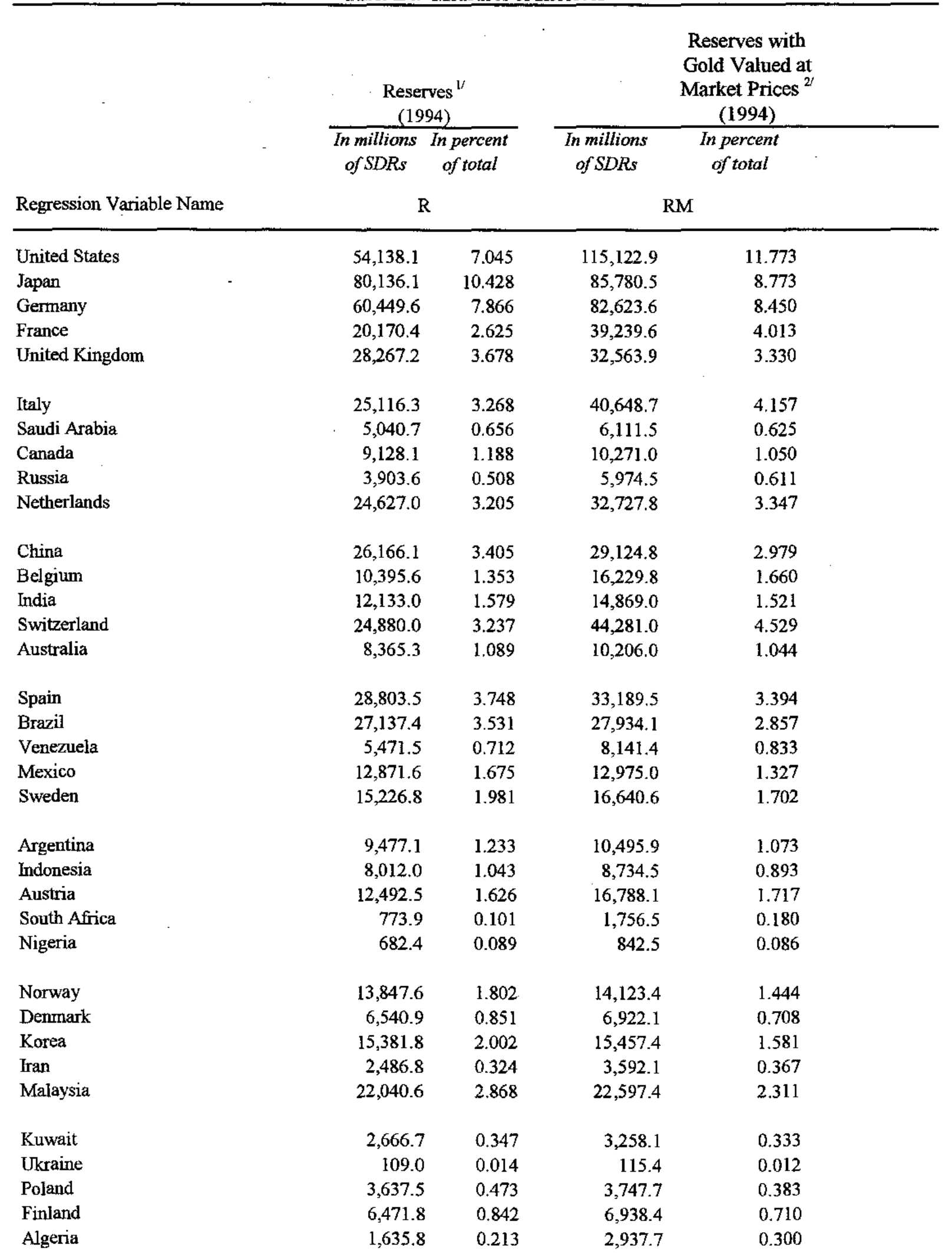


Table I4. Measures of Reserves

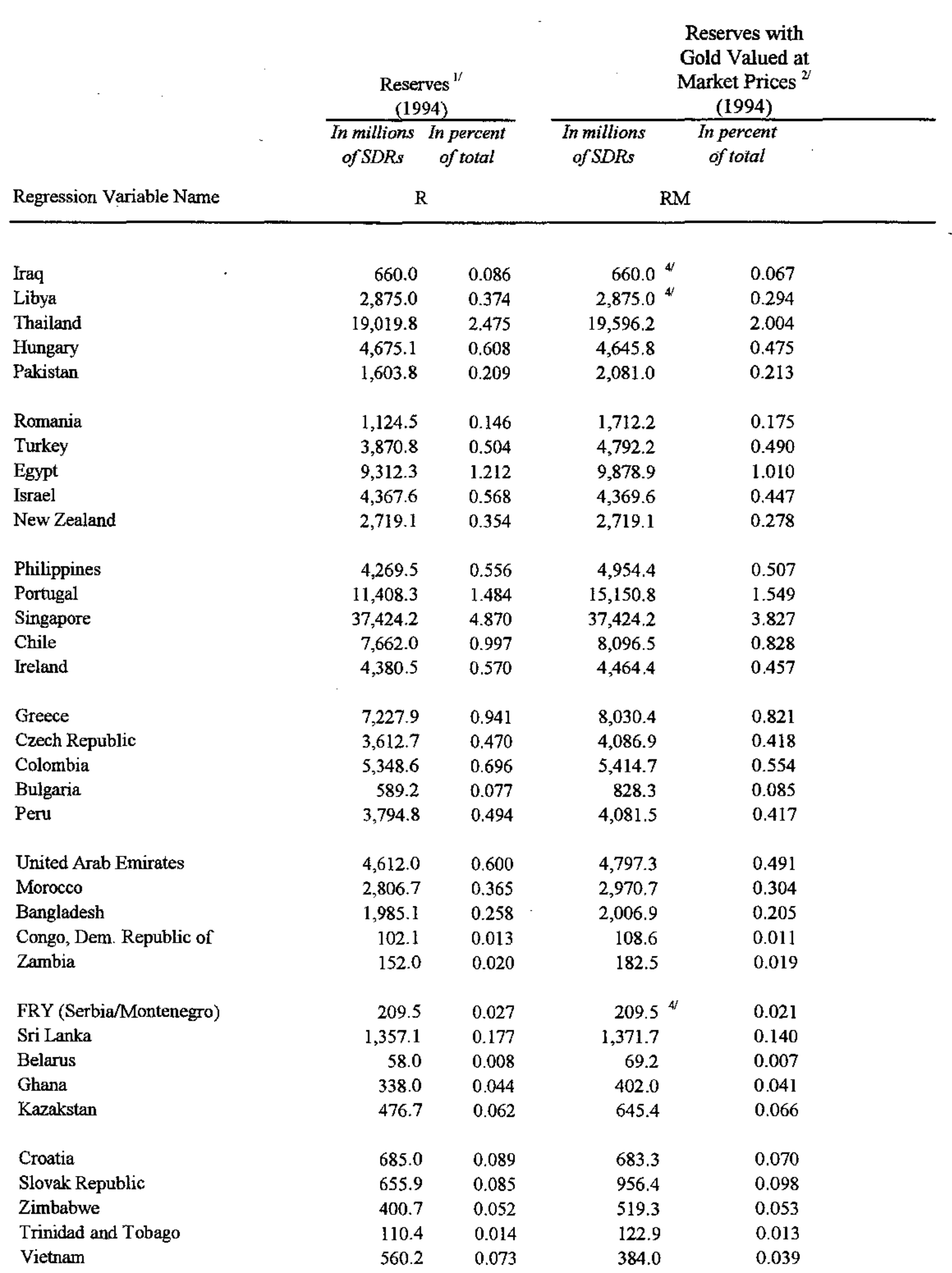


Table I.4. Measures of Reserves

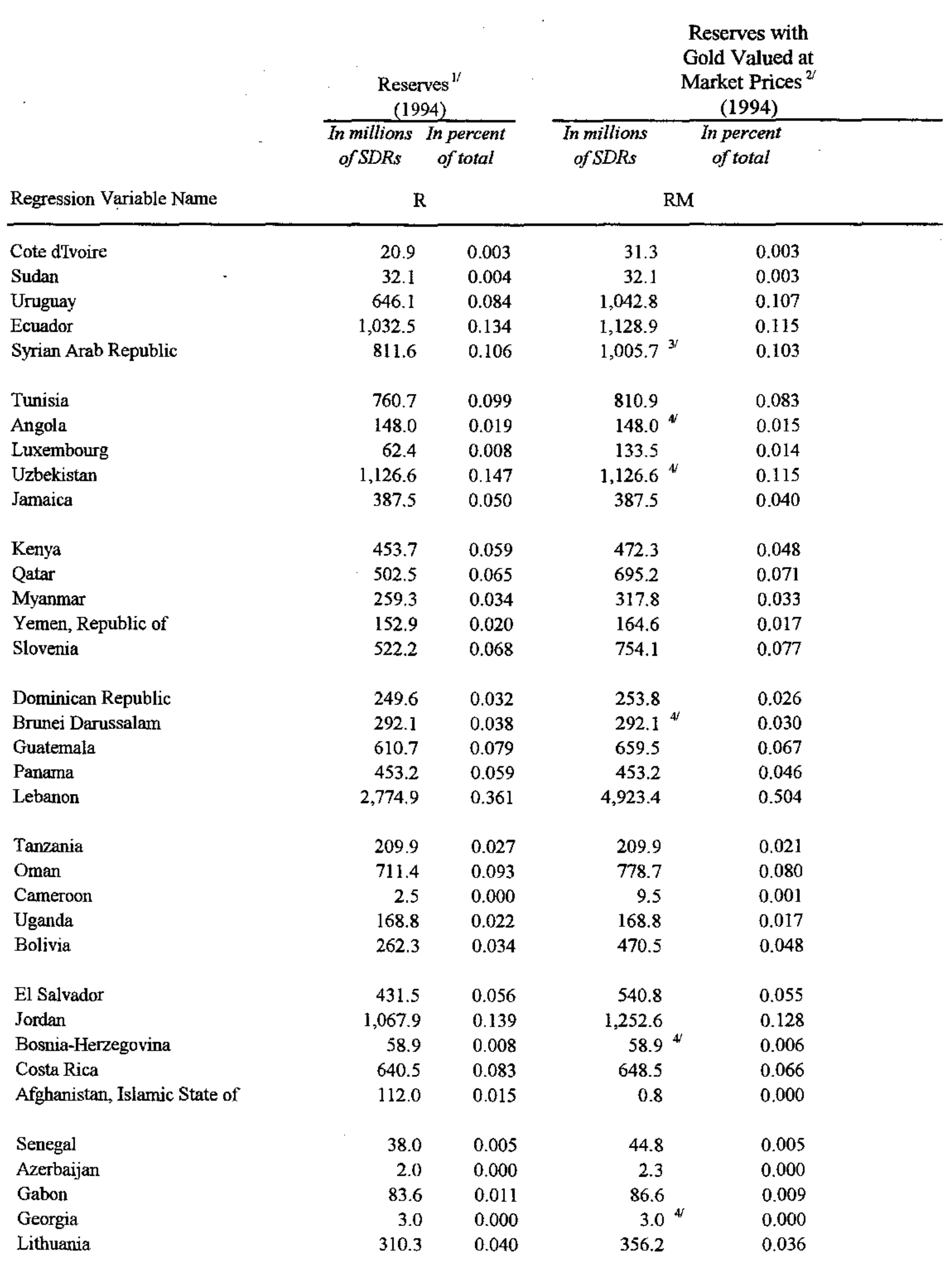


Table 1.4. Measures of Reserves

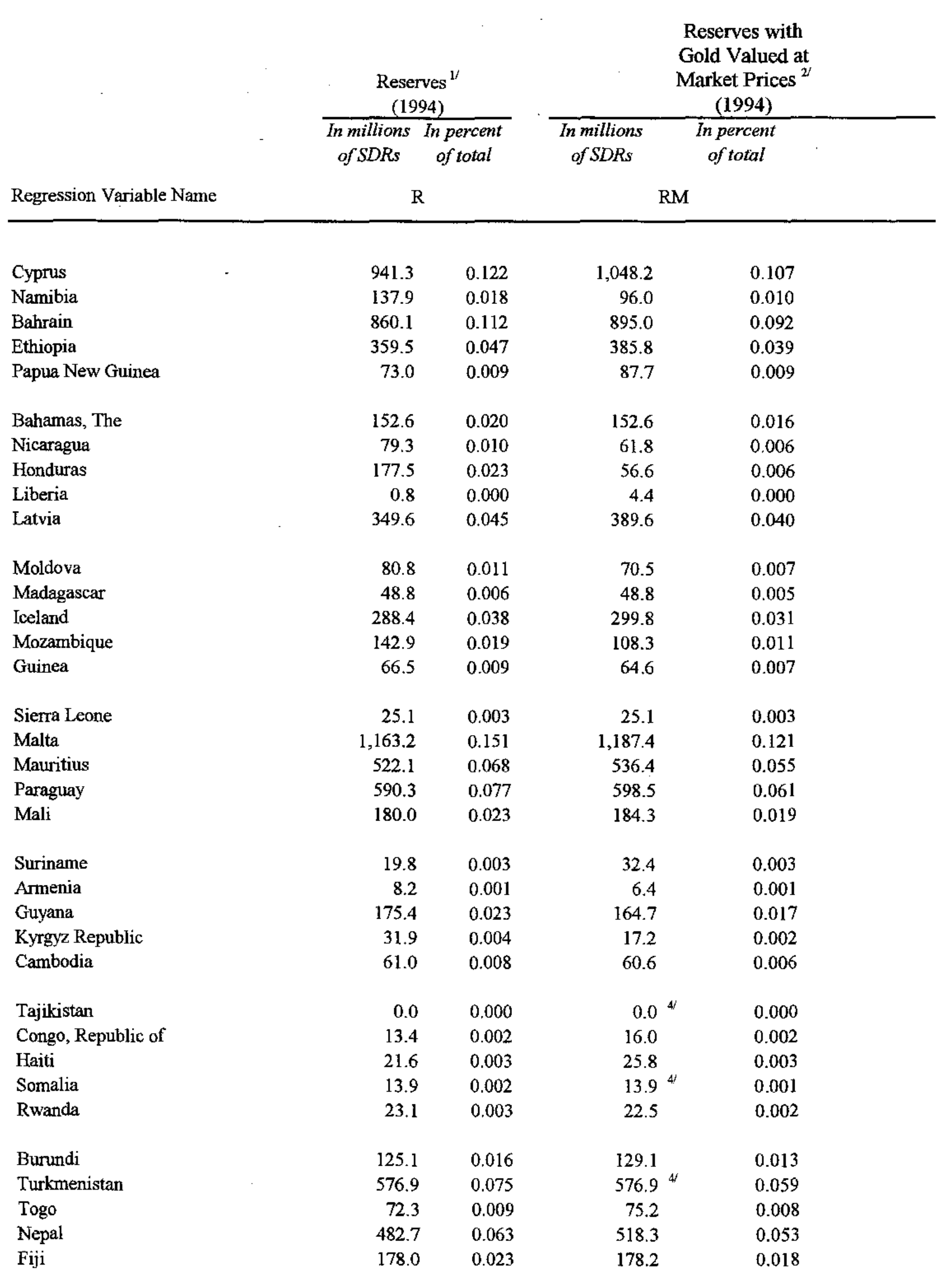


Table I.4. Measures of Reserves

\begin{tabular}{|c|c|c|c|c|}
\hline \multirow[b]{3}{*}{ Regression Variable Name } & \multicolumn{2}{|c|}{$\begin{array}{c}\text { Reserves }^{1 /} \\
(1994) \\
\end{array}$} & \multicolumn{2}{|c|}{$\begin{array}{l}\text { Reserves with } \\
\text { Gold Valued at } \\
\text { Market Prices }{ }^{2 /} \\
\text { (1994) }\end{array}$} \\
\hline & $\begin{array}{l}\text { In millions } \\
\text { of SDRs }\end{array}$ & $\begin{array}{l}\text { In percent } \\
\text { of total }\end{array}$ & $\begin{array}{l}\text { In millions } \\
\text { of SDRs }\end{array}$ & $\begin{array}{l}\text { cent } \\
\text { tal }\end{array}$ \\
\hline & \multicolumn{2}{|c|}{$\mathrm{R}$} & \multicolumn{2}{|c|}{$\mathrm{RM}$} \\
\hline Malawi & 27.7 & 0.004 & 30.7 & 0.003 \\
\hline Macedonia, FYR & 92.9 & 0.012 & 98.7 & 0.010 \\
\hline Barbados & 127.3 & 0.017 & 127.3 & 0.013 \\
\hline Niger & 85.8 & 0.011 & 88.4 & 0.009 \\
\hline Estonia & 288.9 & 0.038 & 290.7 & 0.030 \\
\hline Mauritania & 30.3 & 0.004 & 32.7 & 0.003 \\
\hline Botswana & $3,077.3$ & 0.400 & $3,077.3$ & 0.315 \\
\hline Benin & 105.0 & 0.014 & 107.6 & 0.011 \\
\hline Burkina Faso & 172.8 & 0.022 & 175.4 & 0.018 \\
\hline Chad & 67.1 & 0.009 & 69.7 & 0.007 \\
\hline Central African Republic & 126.4 & 0.016 & 129.0 & 0.013 \\
\hline Lao, People's Dem. Republic & 44.9 & 0.006 & 44.9 & 0.005 \\
\hline Mongolia & 56.0 & 0.007 & 59.8 & 0.006 \\
\hline Swaziland & 191.7 & 0.025 & 191.7 & 0.020 \\
\hline Albania & 120.2 & 0.016 & 131.7 & 0.013 \\
\hline Lesotho & 224.9 & 0.029 & 224.9 & 0.023 \\
\hline Equatorial Guinea & 0.2 & 0.000 & 0.2 & 0.000 \\
\hline Gambia, The & 72.0 & 0.009 & 72.0 & 0.007 \\
\hline Belize & 26.2 & 0.003 & 26.2 & 0.003 \\
\hline San Marino & 120.8 & 0.016 & $120.8^{4 /}$ & 0.012 \\
\hline Vanuatu & 32.6 & 0.004 & 32.6 & 0.003 \\
\hline Djibouti & 53.7 & 0.007 & 53.7 & 0.005 \\
\hline Eritrea & 108.2 & 0.014 & $108.2^{4}$ & 0.011 \\
\hline St. Lucia & 38.8 & 0.005 & 38.8 & 0.004 \\
\hline Guinea-Bissau & 4.7 & 0.001 & 12.8 & 0.001 \\
\hline Antigua and Barbuda & 29.1 & 0.004 & 29.1 & 0.003 \\
\hline Grenada & 20.3 & 0.003 & 20.3 & 0.002 \\
\hline Samoa & 35.6 & 0.005 & 35.6 & 0.004 \\
\hline Solomon Islands & 15.7 & 0.002 & 15.7 & 0.002 \\
\hline Cape Verde & 35.8 & 0.005 & 35.8 & 0.004 \\
\hline Comoros & 29.0 & 0.004 & 28.9 & 0.003 \\
\hline St. Kitts and Nevis & 21.9 & 0.003 & 21.9 & 0.002 \\
\hline Seychelles & 19.5 & 0.003 & 19.5 & 0.002 \\
\hline St. Vincent and the Grenadines & 22.1 & 0.003 & 22.1 & 0.002 \\
\hline Dominica & 11.1 & 0.001 & 11.1 & 0.001 \\
\hline
\end{tabular}


Table I.4. Measures of Reserves

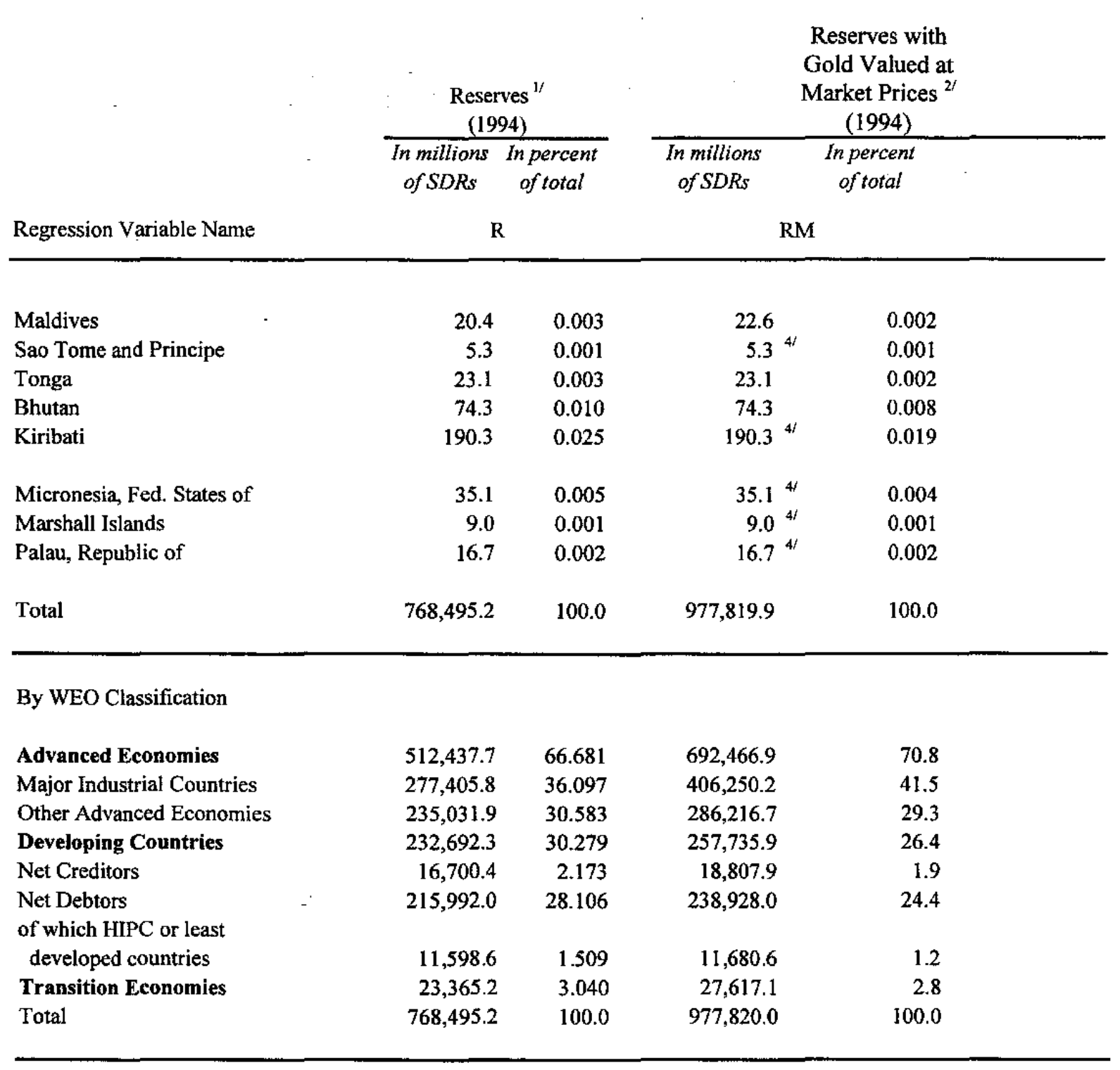

1/ Source: International Financial Statistics; twelve-month average of gold (valued at SDR 35 per fine ounce) and foreign exchange reserves, including SDR holdings, reserve positions in the Fund, and ECUs for 1994. 2/ Source: International Financial Statistics; twelve-month average of gold (valued at market prices in 1994, with monthly prices ranging from SDR 261.02 per fine ounce to SDR 273.82 per fine ounce) and foreign exchange reserves, including SDR holdings, reserve position in the Fund and ECUs, for 1994.

3/ Reserves are calculated by subtracting gold reserves times $\$ 35$ and adding gold reserves times the average market price (in SDRs) for 1994 to the reserves data shown in the first column.

4/ Same as reserves shown in first column. 
Table L.5. Current Account Data

\begin{tabular}{|c|c|c|c|c|c|c|c|}
\hline \multirow[b]{3}{*}{ Regression Variable Name } & & \multicolumn{2}{|c|}{$\begin{array}{c}\text { Current } \\
\text { Receipts }{ }^{1 /} \\
(1990-94) \\
\end{array}$} & \multicolumn{2}{|c|}{$\begin{array}{c}\text { Current } \\
\text { Payments }^{2 t} \\
(1990-94) \\
\end{array}$} & \multicolumn{2}{|c|}{$\begin{array}{c}\text { Average of } \\
\text { Current Receipts and } \\
\text { Current Payments } \\
(1990-94)\end{array}$} \\
\hline & & $\begin{array}{l}\text { In millions } \\
\text { of SDRs }\end{array}$ & $\begin{array}{l}\text { In percent } \\
\text { of total }\end{array}$ & $\begin{array}{l}\text { In millions } \\
\text { of SDRs }\end{array}$ & $\begin{array}{c}\text { In percent } \\
\text { of total }\end{array}$ & $\begin{array}{l}\text { In millions } \\
\text { of SDRs }\end{array}$ & $\begin{array}{c}\text { In percent } \\
\text { of total }\end{array}$ \\
\hline & & \multicolumn{2}{|l|}{$\mathrm{C}$} & \multicolumn{2}{|l|}{$\mathrm{P}$} & \multicolumn{2}{|c|}{ TRADE } \\
\hline United States & . & $537,492.8$ & 14.672 & $583,039.9$ & 15.757 & $560,266.3$ & 15.217 \\
\hline Japan & & $341,088.9$ & 9.311 & $268,259.3$ & 7.250 & $304,674.1$ & 8.275 \\
\hline Germany & & $398,394.9$ & 10.875 & $383,642.1$ & 10.368 & $391,018.5$ & 10.620 \\
\hline France & & $250,108.9$ & 6.827 & $245,578.1$ & 6.637 & $247,843.5$ & 6.732 \\
\hline United Kingdom & & $222,245.6$ & 6.067 & $229,428.8$ & 6.200 & $225,837.2$ & 6.134 \\
\hline Italy & & $179,451.1$ & 4.898 & $182,032.0$ & 4.920 & $180,741.5$ & 4.909 \\
\hline Saudi Arabia & & $40,129.2$ & 1.095 & $49,287.9$ & 1.332 & $44,708.5$ & 1.214 \\
\hline Canada & & $124,213.5$ & 3.391 & $138,012.6$ & 3.730 & $131,113.0$ & 3.561 \\
\hline Russia & & $54,084.1$ & 1.476 & $50,121.5$ & 1.355 & $52,102.8$ & 1.415 \\
\hline Netherlands & & $134,236.8$ & 3.664 & $123,819.9$ & 3.346 & $129,028.4$ & 3.504 \\
\hline China & & $62,088.9$ & 1.695 & $58,254.6$ & 1.574 & $60,171.8$ & 1.634 \\
\hline Belgium & & $107,978.3$ & 2.947 & $104,945.3$ & 2.836 & $106,461.8$ & 2.892 \\
\hline India & & $20,639.2$ & 0.563 & $24,557.2$ & 0.664 & $22,598.2$ & 0.614 \\
\hline Switzerland & & $75,508.4$ & 2.061 & $65,732.1$ & 1.776 & $70,620.2$ & 1.918 \\
\hline Australia & & $42,145.1$ & 1.150 & $51,212.4$ & 1.384 & $46,678.8$ & 1.268 \\
\hline Spain & & $81,303.2$ & 2.219 & $88,682.1$ & 2.397 & $84,992.7$ & 2.308 \\
\hline Brazil & & $31,262.4$ & 0.853 & $31,332.6$ & 0.847 & $31,297.5$ & 0.850 \\
\hline Venezuela & & $13,882.7$ & 0.379 & $12,855.1$ & 0.347 & $13,368.9$ & 0.363 \\
\hline Mexico & & $35,729.6$ & 0.975 & $49,934.9$ & 1.350 & $42,832.3$ & 1.163 \\
\hline Sweden & & $54,392.1$ & 1.485 & $56,492.7$ & 1.527 & $55,442.4$ & 1.506 \\
\hline Argentina & & $13,318.7$ & 0.364 & $15,939.4$ & 0.431 & $14,629.0$ & 0.397 \\
\hline Indonesia & & $27,235.7$ & 0.743 & $29,541.4$ & 0.798 & $28,388.5$ & 0.771 \\
\hline Austria & & $56,863.9$ & 1.552 & $56,817.3$ & 1.536 & $56,840.6$ & 1.544 \\
\hline South Africa & & $20,647.6$ & 0.564 & $19,611.3$ & 0.530 & $20,129.4$ & 0.547 \\
\hline Nigeria & & $9,157.4$ & 0.250 & $8,552.8$ & 0.231 & $8,855.1$ & $0.24 \mathrm{I}$ \\
\hline Norway & & $36,394.0$ & 0.993 & $32,974.0$ & 0.891 & $34,684.0$ & 0.942 \\
\hline Denmark & & $48,389.8$ & 1.321 & $45,678.1$ & 1.234 & $47,034.0$ & 1.277 \\
\hline Korea & & $68,655.9$ & 1.874 & $70,997.8$ & 1.919 & $69,826.8$ & 1.897 \\
\hline Iran & & $15,664.7$ & 0.428 & $17,832.3$ & 0.482 & $16,748.5$ & 0.455 \\
\hline Malaysia & & $35,035.4$ & 0.956 & $37,176.3$ & 1.005 & $36,105.8$ & 0.981 \\
\hline Kuwait & & $10,219.4$ & 0.279 & $8,676.8$ & 0.234 & $9,448.1$ & 0.257 \\
\hline Ukraine & & $11,259.9$ & 0.307 & $11,540.0$ & 0.312 & $11,399.9$ & 0.310 \\
\hline Poland & & $18,477.0$ & 0.504 & $19,600.5$ & 0.530 & $19,038.8$ & 0.517 \\
\hline Finland & & $23,389.2$ & 0.638 & $25,709.1$ & 0.695 & $24,549.1$ & 0.667 \\
\hline Algeria & & $9,263.0$ & 0.253 & $8,649.2$ & 0.234 & $8,956.1$ & 0.243 \\
\hline
\end{tabular}


Table I.5. Current Account Data

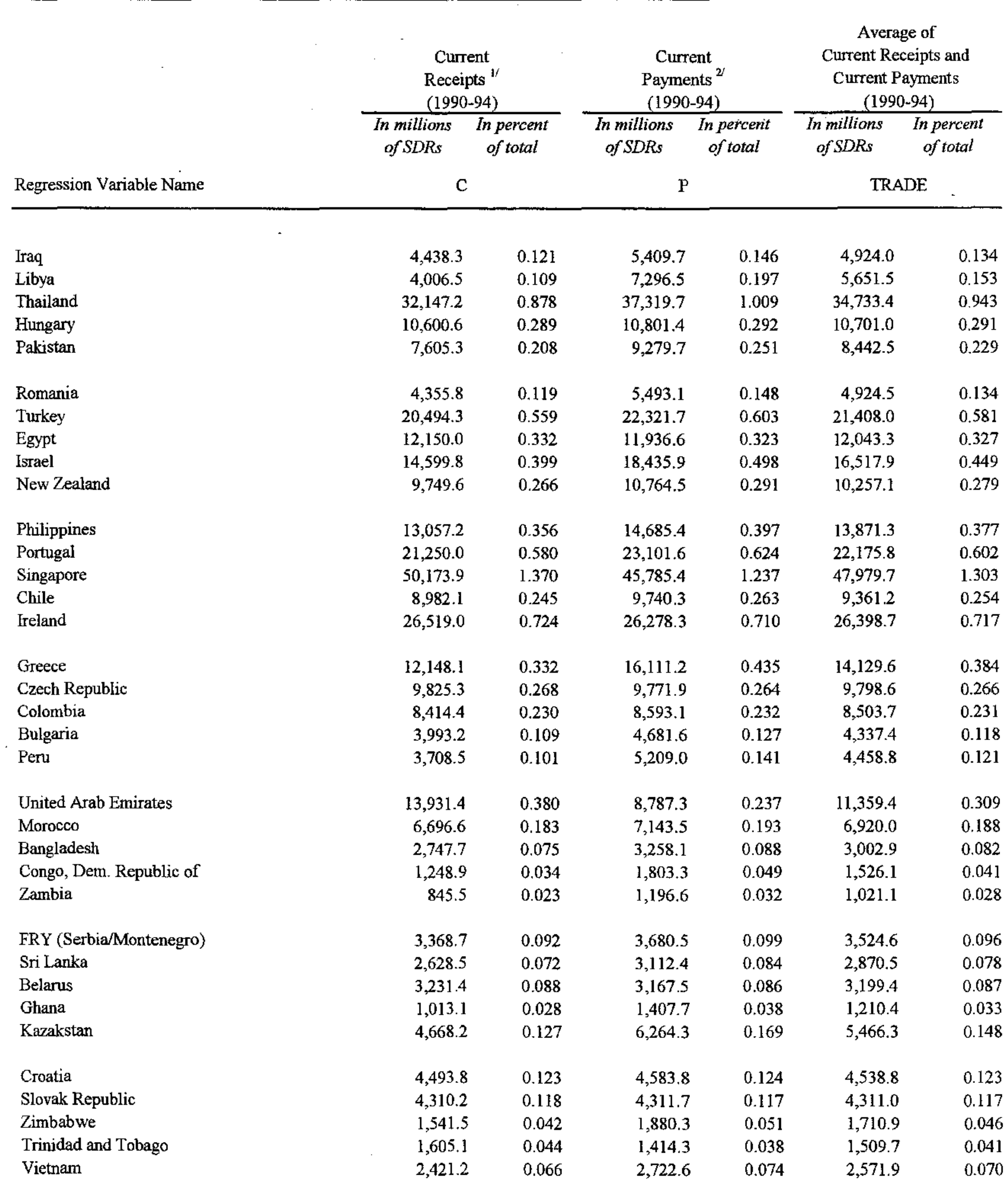


Table L.5. Current Account Data

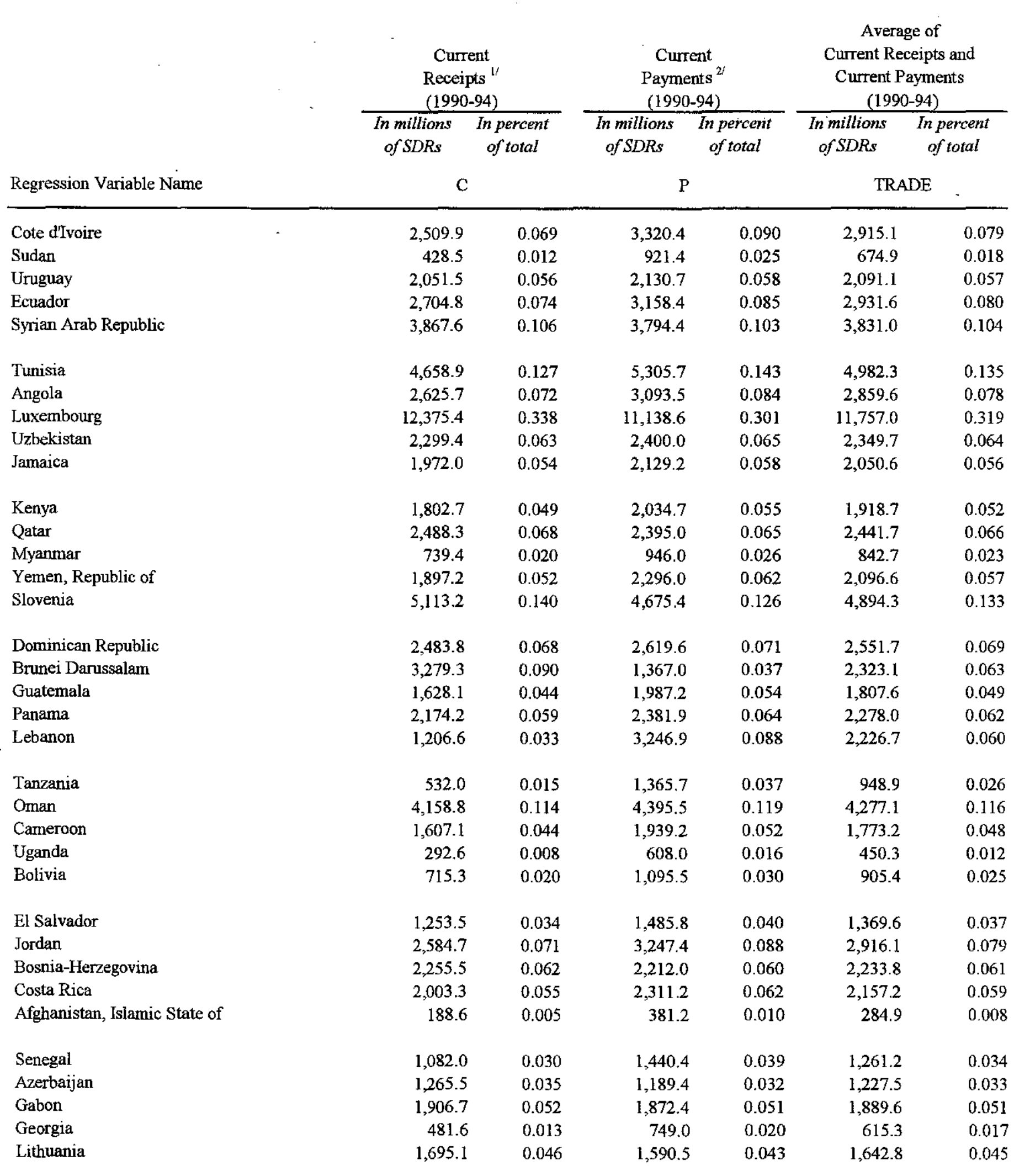


Table L5. Current Account Data

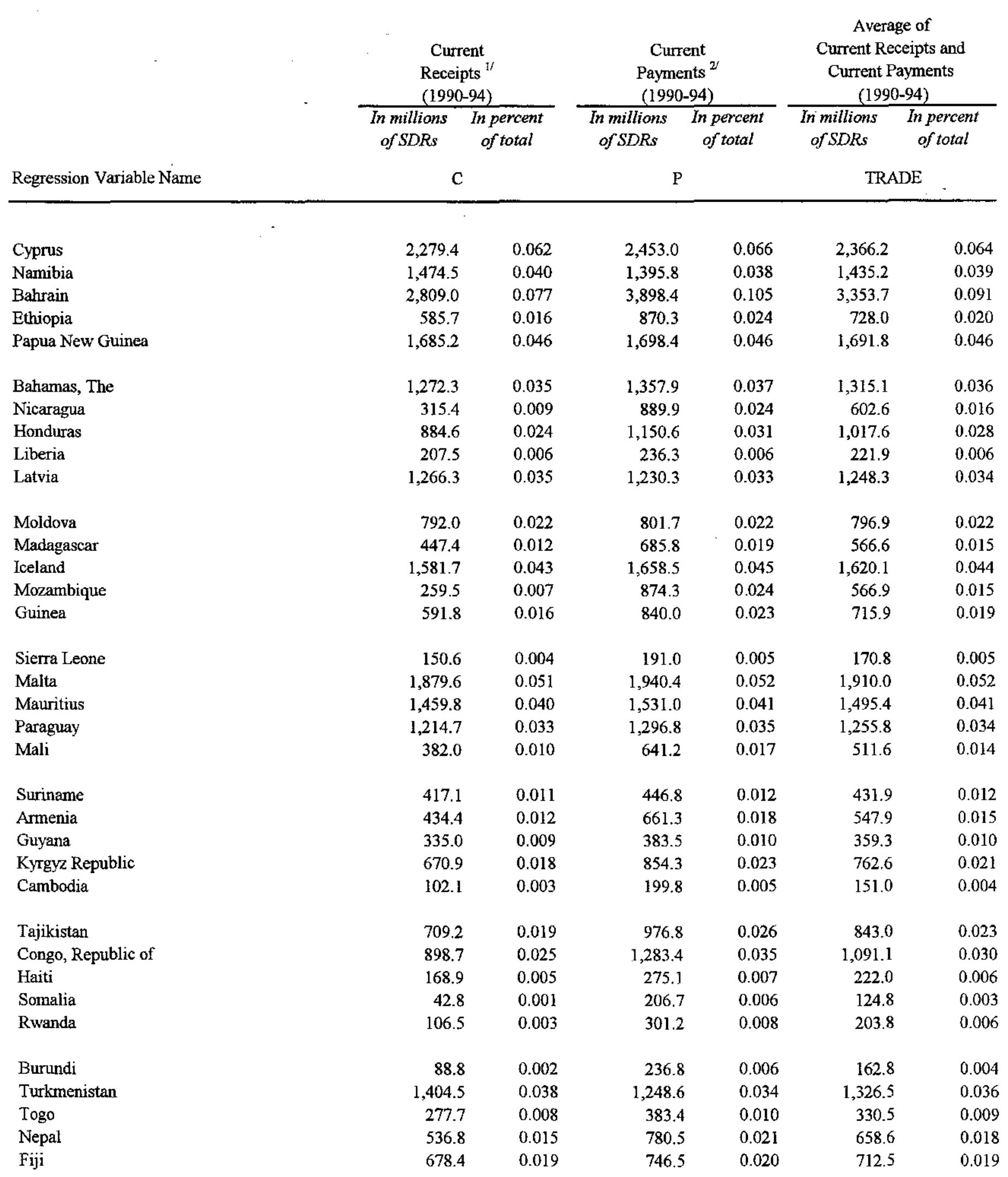


Table L.5. Current Account Data

\begin{tabular}{|c|c|c|c|c|c|c|c|}
\hline \multirow[b]{3}{*}{ Regression Variable Name } & & \multicolumn{2}{|c|}{$\begin{array}{c}\text { Current } \\
\text { Receipts }{ }^{1 /} \\
(1990-94) \\
\end{array}$} & \multicolumn{2}{|c|}{$\begin{array}{c}\text { Current } \\
\text { Payments }{ }^{2 \prime} \\
(1990-94)\end{array}$} & \multicolumn{2}{|c|}{$\begin{array}{c}\text { Average of } \\
\text { Current Receipts and } \\
\text { Current Payments } \\
(1990-94) \\
\end{array}$} \\
\hline & & $\begin{array}{l}\text { In millions } \\
\text { of SDRs }\end{array}$ & $\begin{array}{c}\text { In percent } \\
\text { of total }\end{array}$ & $\begin{array}{c}\text { In millions } \\
\text { of SDRs }\end{array}$ & $\begin{array}{c}\text { In percent } \\
\text { of total }\end{array}$ & $\begin{array}{l}\text { In millions } \\
\text { of SDRs }\end{array}$ & $\begin{array}{c}\text { In percent } \\
\text { of total }\end{array}$ \\
\hline & & \multicolumn{2}{|l|}{$\mathrm{C}$} & \multicolumn{2}{|c|}{$\mathrm{P}$} & \multicolumn{2}{|c|}{ TRADE } \\
\hline Malawi & . & 362.8 & 0.010 & 592.7 & 0.016 & 477.7 & 0.013 \\
\hline Macedonia, FYR & & 972.0 & 0.027 & $1,087.5$ & 0.029 & $1,029.8$ & 0.028 \\
\hline Barbados & & 687.7 & 0.019 & 642.1 & 0.017 & 664.9 & 0.018 \\
\hline Niger & & 301.7 & 0.008 & 490.4 & 0.013 & 396.1 & 0.011 \\
\hline Estonia & & 801.3 & 0.022 & 847.8 & 0.023 & 824.5 & 0.022 \\
\hline Mauritania & & 336.4 & 0.009 & 464.5 & 0.013 & 400.5 & 0.011 \\
\hline Botswana & & $1,782.1$ & 0.049 & $1,702.8$ & 0.046 & $1,742.5$ & 0.047 \\
\hline Benin & & 394.3 & 0.011 & 480.7 & 0.013 & 437.5 & 0.012 \\
\hline Burkina Faso & & 355.7 & 0.010 & 546.8 & 0.015 & 451.3 & 0.012 \\
\hline Chad & & 177.9 & 0.005 & 366.3 & 0.010 & 272.1 & 0.007 \\
\hline Central African Republic & & 141.8 & 0.004 & 272.1 & 0.007 & 207.0 & 0.006 \\
\hline Lao, People's Dem. Republic & & 168.7 & 0.005 & 275.7 & 0.007 & 222.2 & 0.006 \\
\hline Mongolia & & 297.7 & 0.008 & 437.8 & 0.012 & 367.8 & 0.010 \\
\hline Swaziland & & 658.6 & 0.018 & 754.2 & 0.020 & 706.4 & 0.019 \\
\hline Albania & & 253.4 & 0.007 & 439.1 & 0.012 & 346.2 & 0.009 \\
\hline Lesotho & & 435.1 & 0.012 & 661.3 & 0.018 & 548.2 & 0.015 \\
\hline Equatorial Guinea & & 36.9 & 0.001 & 80.8 & 0.002 & 58.9 & 0.002 \\
\hline Gambia, The & & 168.6 & 0.005 & 184.7 & 0.005 & 176.6 & 0.005 \\
\hline Belize & & 210.6 & 0.006 & 238.1 & 0.006 & 224.4 & 0.006 \\
\hline San Marino & & 549.0 & 0.015 & 502.7 & 0.014 & 525.9 & 0.014 \\
\hline Vanuatu & & 84.4 & 0.002 & 103.6 & 0.003 & 94.0 & 0.003 \\
\hline Djibouti & & 141.0 & 0.004 & 220.5 & 0.006 & 180.7 & 0.005 \\
\hline Eritrea & & 163.8 & 0.004 & 169.9 & 0.005 & 166.9 & 0.005 \\
\hline St. Lucia & & 236.4 & 0.006 & 276.8 & 0.007 & 256.6 & 0.007 \\
\hline Guinea-Bissau & & 31.9 & 0.001 & 76.2 & 0.002 & 54.0 & 0.001 \\
\hline Antigua and Barbuda & & 353.2 & 0.010 & 381.9 & 0.010 & 367.6 & 0.010 \\
\hline Grenada & & 98.6 & 0.003 & 114.0 & 0.003 & 106.3 & 0.003 \\
\hline Samoa & & 61.1 & 0.002 & 85.5 & 0.002 & 73.3 & 0.002 \\
\hline Solomon Islands & & 110.5 & 0.003 & 1512 & 0.004 & 130.9 & 0.004 \\
\hline Cape Verde & & 93.4 & 0.003 & 136.4 & 0.004 & 114.9 & 0.003 \\
\hline Comoros & & 61.8 & 0.002 & 76.1 & 0.002 & 68.9 & 0.002 \\
\hline St. Kitts and Nevis & & 88.8 & 0.002 & 109.0 & 0.003 & 98.9 & 0.003 \\
\hline Seychelles & & 181.7 & 0.005 & 208.9 & 0.006 & 195.3 & 0.005 \\
\hline St. Vincent and the Grenadines & & 99.0 & 0.003 & 123.5 & 0.003 & 111.3 & 0.003 \\
\hline Dominica & & 77.3 & 0.002 & 101.0 & 0.003 & 89.2 & 0.002 \\
\hline
\end{tabular}


Table I.5. Current Account Data

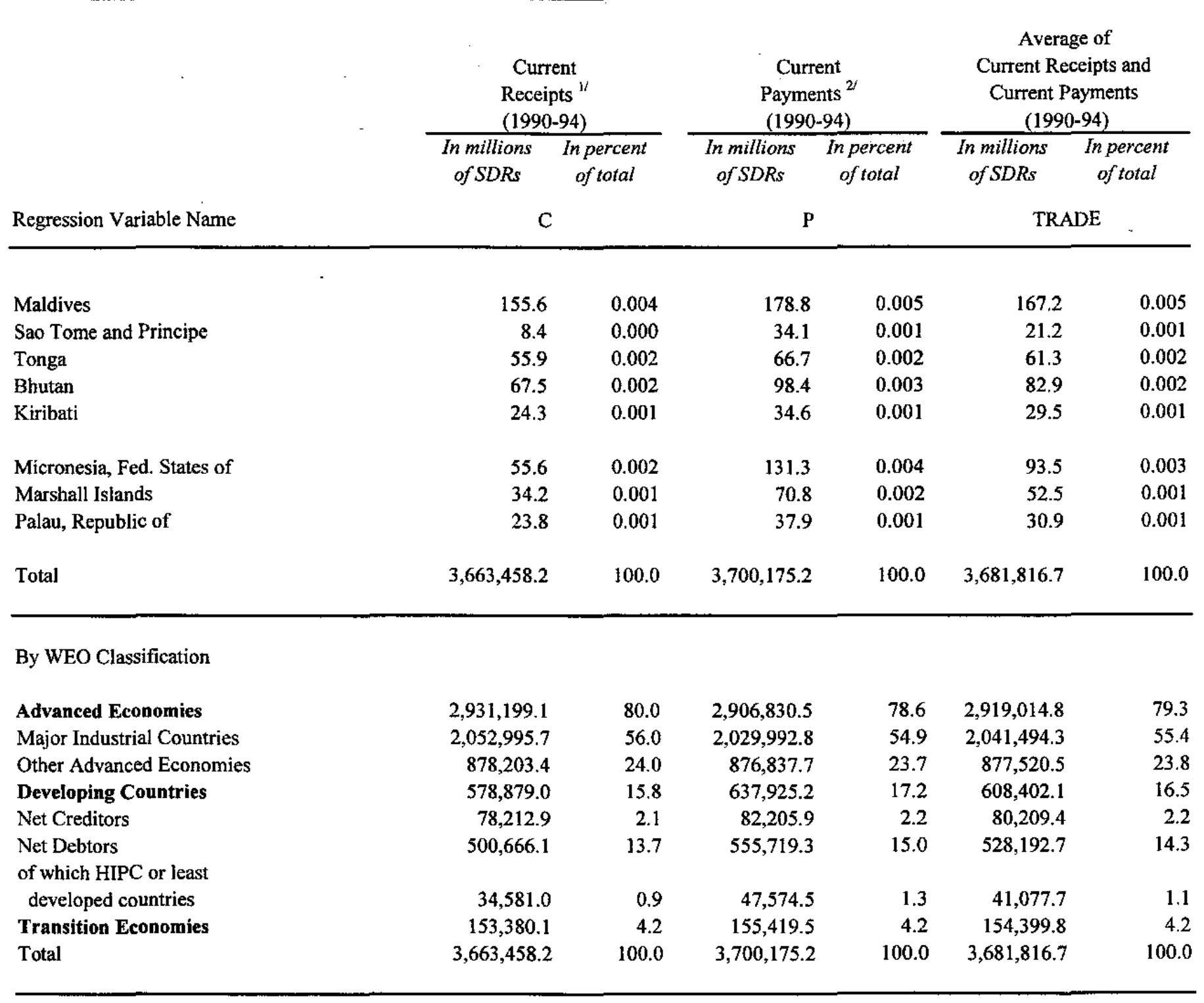

1/ Source: Balance of Payments database; 1990-94 average of the sum of goods(exports f.o.b.), services(credit), income(credit), and private current transfers(credit) divided by the average SDR value for the same years.

2/ Source: Balance of Payments database; 1990-94 average of the sum of goods(imports f.o.b.), services(debit), income(debit), and private current transfers(debit); divided by the average SDR value for the same years. 
Table I.6. Variability Measures

\begin{tabular}{|c|c|c|c|c|c|c|}
\hline \multirow[b]{3}{*}{ Regression Variable Name } & & \multicolumn{2}{|c|}{$\begin{array}{c}\text { Variability of } \\
\text { Current Receipts }{ }^{\text {1/ }} \\
(1982-94)\end{array}$} & \multicolumn{2}{|c|}{$\begin{array}{l}\text { Variability of } \\
\text { External Receipts } \\
(1982-94)\end{array}$} & \multirow{2}{*}{ 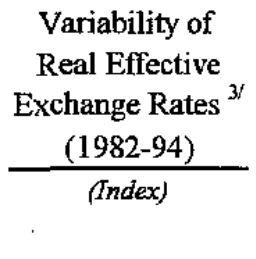 } \\
\hline & - & $\begin{array}{l}\text { In millions of } \\
\text { SDRs }\end{array}$ & $\begin{array}{c}\text { In percent of } \\
\text { total }\end{array}$ & $\begin{array}{l}\text { In millions of } \\
\quad S D R s\end{array}$ & $\begin{array}{l}\text { In percent of } \\
\text { total }\end{array}$ & \\
\hline & & \multicolumn{2}{|c|}{$\mathrm{VC}$} & \multicolumn{2}{|c|}{ VCK } & VREC \\
\hline United States & & $25,272.4$ & 14.589 & $32,940.6$ & 10.240 & 1.040 \\
\hline Japan & & $9,844.2$ & 5.683 & $28,314.2$ & 8.802 & 1.059 \\
\hline Germany & & $13,981.5$ & 8.071 & $18,698.9$ & 5.813 & 1.022 \\
\hline France & & $8,361.5$ & 4.827 & $26,683.6$ & 8.295 & 1.018 \\
\hline United Kingdom & & $6,337.4$ & 3.658 & $32,560.2$ & 10.122 & 1.029 \\
\hline Italy & & $6,039.5$ & 3.486 & $12,008.8$ & 3.733 & 1.032 \\
\hline Saudi Arabia & & $3,827.4$ & 2.209 & $3,808.5$ & 1.184 & 1.053 \\
\hline Canada & & $7,084.9$ & 4.090 & $8,931.0$ & 2.776 & 1.036 \\
\hline Russia & & $7,762.8$ & 4.481 & $8,053.1^{4}$ & 2.503 & 1.000 \\
\hline Netherlands & & $2,915.2$ & 1.683 & $5,699.8$ & 1.772 & 1.018 \\
\hline China & & $1,850.6$ & 1.068 & $4,072.1$ & 1.266 & 1.079 \\
\hline Belgium & & $3,541.1$ & 2.044 & $25,897.6$ & 8.051 & 1.016 \\
\hline India & & 880.6 & 0.508 & $1,253.0$ & 0.390 & 1.038 \\
\hline Switzerland & & $1,837.9$ & 1.061 & $2,760.0$ & 0.858 & 1.028 \\
\hline Australia & & $2,154.4$ & 1.244 & $3,362.6$ & 1.045 & 1.066 \\
\hline Spain & & $2,692.2$ & 1.554 & $6,159.2$ & 1.915 & 1.031 \\
\hline Brazil & & $3,260.7$ & 1.882 & $4,232.4$ & 1.316 & 1.113 \\
\hline Venezuela & & $2,213.1$ & 1.278 & $2,447.3$ & 0.761 & 1.064 \\
\hline Mexico & - & $3,091.8$ & 1.785 & $4,790.4$ & 1.489 & 1.076 \\
\hline Sweden & & $1,555.1$ & 0.898 & $8,969.5$ & 2.788 & 1.040 \\
\hline Argentina & & $1,158.5$ & 0.669 & $2,273.3$ & 0.707 & 1.095 \\
\hline Indonesia & & $1,918.9$ & 1.108 & $1,527.3$ & 0.475 & 1.054 \\
\hline Austria & & $1,778.7$ & 1.027 & $1,948.7$ & 0.606 & 1.010 \\
\hline South Africa & & 461.0 & 0.266 & $1,360.1$ & 0.423 & 1.079 \\
\hline Nigeria & & $2,211.5$ & 1.277 & $2,100.2$ & 0.653 & 1.319 \\
\hline Norway & & $2,117.5$ & 1.222 & $2,022.9$ & 0.629 & 1.012 \\
\hline Denmark & & $1,003.4$ & 0.579 & $2,831.0$ & 0.880 & 1.018 \\
\hline Korea & & $2,504.9$ & 1.446 & $3,457.5$ & 1.075 & 1.054 \\
\hline Iran & & $2,140.4$ & 1.236 & $1,995.7$ & 0.620 & 1.269 \\
\hline Malaysia & & $1,752.5$ & 1.012 & $1,892.6$ & 0.588 & 1.045 \\
\hline Kuwait & & $2,771.3$ & 1.600 & $2,818.2$ & 0.876 & 1.000 \\
\hline Ukraine & & $1,616.2$ & 0.933 & $1,713.8^{4 /}$ & 0.533 & 1.000 \\
\hline Poland & & 976.9 & 0.564 & $1,043.0$ & 0.324 & 1.107 \\
\hline Finland & & $1,249.5$ & 0.721 & $3,093.7$ & 0.962 & 1.037 \\
\hline Algeria & & $1,422.7$ & 0.821 & $1,482.9$ & 0.461 & 1.084 \\
\hline
\end{tabular}


Table I.6. Variability Measures

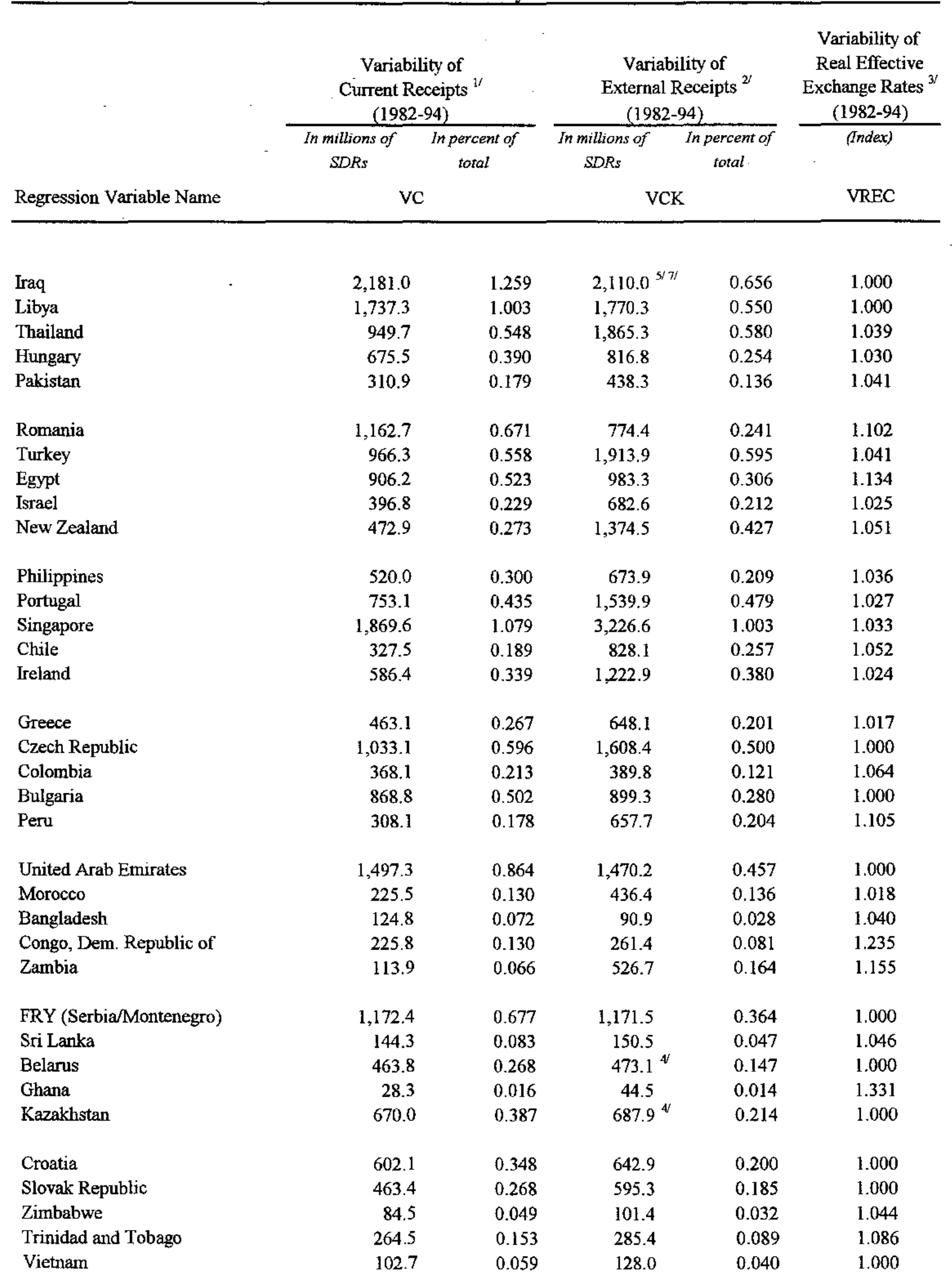


Table L.6. Variability Measures

\begin{tabular}{|c|c|c|c|c|c|}
\hline \multirow[b]{3}{*}{ Regression Variable Name } & \multicolumn{2}{|c|}{$\begin{array}{c}\text { Variability of } \\
\text { Current Receipts }{ }^{1 /} \\
(1982-94)\end{array}$} & \multicolumn{2}{|c|}{$\begin{array}{c}\text { Variability of } \\
\text { External Receipts }{ }^{2 /} \\
(1982-94) \\
\end{array}$} & \multirow{2}{*}{ 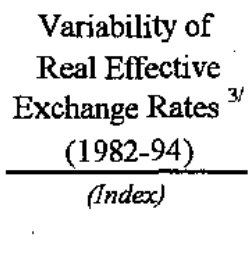 } \\
\hline & $\begin{array}{l}\text { In millions of } \\
\text { SDRs }\end{array}$ & $\begin{array}{c}\text { In percent of } \\
\text { total }\end{array}$ & $\begin{array}{l}\text { In millions of } \\
\text { SDRs }\end{array}$ & $\begin{array}{c}\text { In percent of } \\
\text { total }\end{array}$ & \\
\hline & \multicolumn{2}{|c|}{ VC } & \multicolumn{2}{|c|}{ VCK } & VREC \\
\hline Cote dTvoire & 162.8 & 0.094 & 169.1 & 0.053 & 1.053 \\
\hline Sudan & 205.2 & 0.118 & 278.7 & 0.087 & 1.667 \\
\hline Uruguay & 88.8 & 0.051 & 207.4 & 0.064 & 1.052 \\
\hline Ecuador & 298.9 & 0.173 & 201.1 & 0.063 & 1.070 \\
\hline Syrian Arab Republic & 499.8 & 0.289 & 451.5 & 0.140 & 1.307 \\
\hline Tunisia & 184.8 & 0.107 & 188.8 & 0.059 & 1.033 \\
\hline Angola & 334.4 & 0.193 & 387.6 & 0.120 & 1.000 \\
\hline Luxembourg & 394.2 & 0.228 & 853.3 & 0.265 & 1.005 \\
\hline Uzbekistan & 330.0 & 0.191 & $341.5^{4}$ & 0.106 & 1.000 \\
\hline Jamaica & 99.6 & 0.057 & 186.2 & 0.058 & 1.085 \\
\hline Kenya & 105.5 & 0.061 & 126.4 & 0.039 & 1.032 \\
\hline Qatar & 390.0 & 0.225 & 388.3 & 0.121 & 1.000 \\
\hline Myanmar & 60.1 & 0.035 & 103.6 & 0.032 & 1.055 \\
\hline Yemen, Republic of & 322.0 & 0.186 & 333.2 & 0.104 & 1.000 \\
\hline Slovenia & 421.5 & 0.243 & 433.9 & 0.135 & 1.000 \\
\hline Dominican Republic & 167.3 & 0.097 & 240.3 & 0.075 & 1.090 \\
\hline Brunei Darussalam & 454.1 & 0.262 & 449.5 & 0.140 & 1.000 \\
\hline Guatemala & 66.2 & 0.038 & 128.8 & 0.040 & 1.090 \\
\hline Panama & 200.7 & 0.116 & 249.6 & 0.078 & 1.016 \\
\hline Lebanon & 177.6 & 0.103 & 224.1 & 0.070 & 1.000 \\
\hline Tanzania & 58.3 & 0.034 & 87.8 & 0.027 & 1.175 \\
\hline Oman & 593.4 & 0.343 & 529.5 & 0.165 & 1.000 \\
\hline Cameroon & 164.7 & 0.095 & 253.8 & 0.079 & 1.050 \\
\hline Uganda & 38.0 & 0.022 & 68.0 & 0.021 & 1.177 \\
\hline Bolivia & 80.5 & 0.046 & 88.7 & 0.028 & 1.255 \\
\hline El Salvador & 58.7 & 0.034 & 82.4 & 0.026 & 1.068 \\
\hline Jordan & 165.4 & 0.095 & 301.9 & 0.094 & 1.042 \\
\hline Bosnia-Herzegovina & 127.6 & 0.074 & $1,969.3$ & 0.612 & 1.000 \\
\hline Costa Rica & 81.2 & 0.047 & 85.2 & 0.026 & 1.036 \\
\hline Afghanistan, Islamic State of & 29.9 & 0.017 & $38.3^{7}$ & 0.012 & 1.000 \\
\hline Senegal & 58.8 & 0.034 & 40.4 & 0.013 & 1.047 \\
\hline Azerbaijan & 181.6 & 0.105 & $184.4^{4 /}$ & 0.057 & 1.000 \\
\hline Gabon & 277.7 & 0.160 & 239.3 & 0.074 & 1.066 \\
\hline Georgia & 69.1 & 0.040 & $70.8^{4 i}$ & 0.022 & 1.000 \\
\hline Lithuania & 243.3 & 0.140 & $252.7^{4}$ & 0.079 & 1.000 \\
\hline
\end{tabular}


Table L6. Variability Measures

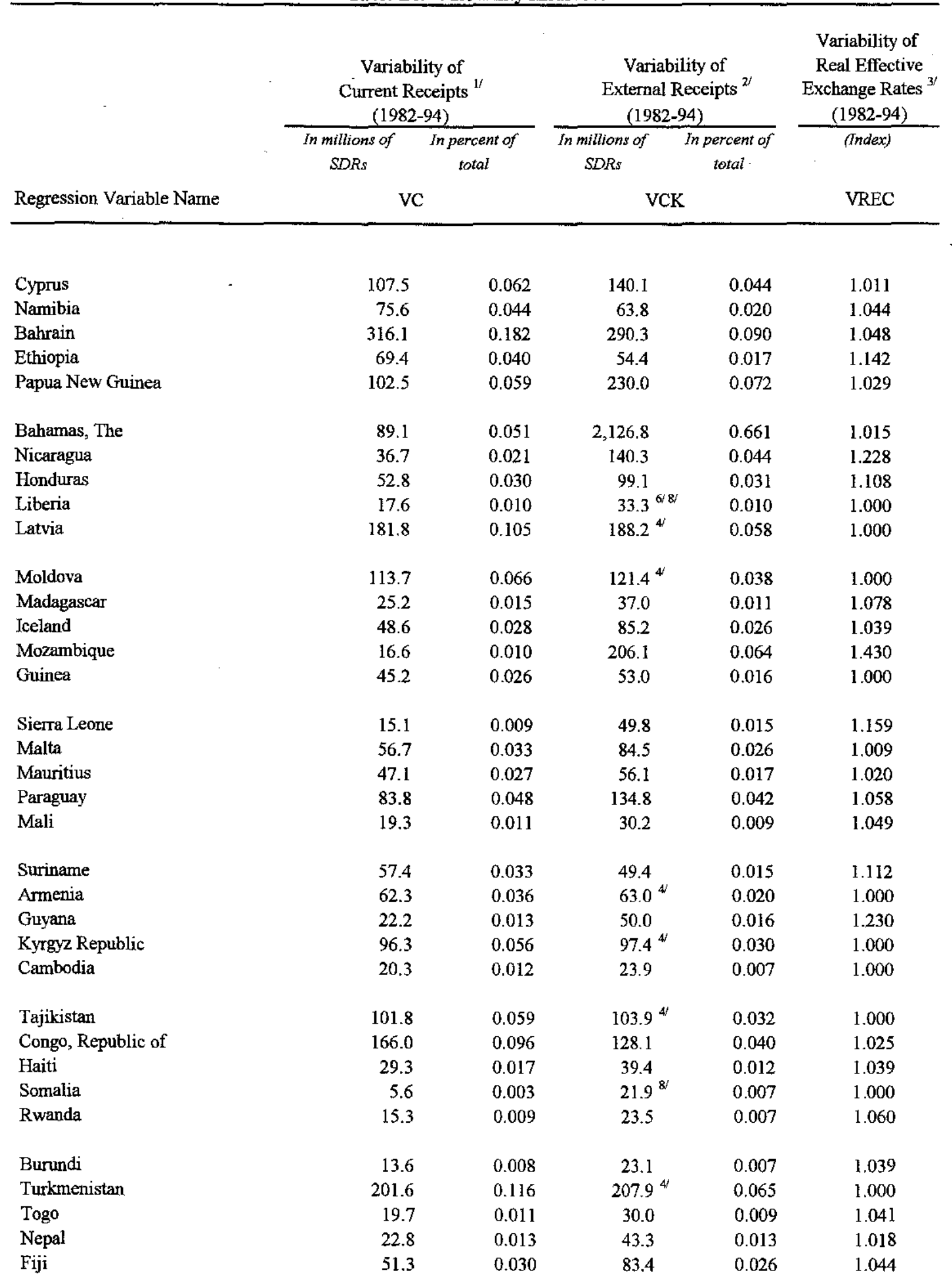


Table L6. Variability Measures

\begin{tabular}{|c|c|c|c|c|c|}
\hline \multirow[b]{3}{*}{ Regression Variable Name } & \multicolumn{2}{|c|}{$\begin{array}{c}\text { Variability of } \\
\text { Current Receipts } \\
(1982-94)\end{array}$} & \multicolumn{2}{|c|}{$\begin{array}{c}\text { Variability of } \\
\text { External Receipts } \\
(1982-94)\end{array}$} & \multirow{2}{*}{ 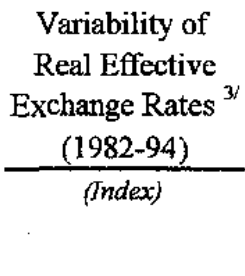 } \\
\hline & $\begin{array}{l}\text { In millions of } \\
\text { SDRs }\end{array}$ & $\begin{array}{c}\text { In percent of } \\
\text { total }\end{array}$ & $\begin{array}{l}\text { In millions of } \\
\text { SDRs }\end{array}$ & $\begin{array}{c}\text { In percent of } \\
\text { total }\end{array}$ & \\
\hline & \multicolumn{2}{|c|}{$\mathrm{VC}$} & \multicolumn{2}{|c|}{ VCK } & VREC \\
\hline Malawi & 39.6 & 0.023 & 58.0 & 0.018 & 1.034 \\
\hline Macedonia, FYR & 64.8 & 0.037 & 74.3 & 0.023 & 1.000 \\
\hline Barbados & 74.8 & 0.043 & 83.7 & 0.026 & 1.027 \\
\hline Niger & 37.0 & 0.021 & 47.5 & 0.015 & 1.020 \\
\hline Estonia & 115.0 & 0.066 & $129.8^{4 /}$ & 0.040 & 1.000 \\
\hline Mauritania & 20.9 & 0.012 & 55.3 & 0.017 & 1.034 \\
\hline Botswana & 117.6 & 0.068 & 145.0 & 0.045 & 1.026 \\
\hline Benin & 40.0 & 0.023 & 46.8 & 0.015 & 1.000 \\
\hline Burkina Faso & 20.8 & 0.012 & 73.7 & 0.023 & 1.026 \\
\hline Chad & 19.5 & 0.011 & 24.4 & 0.008 & 1.062 \\
\hline Central African Republic & 15.4 & 0.009 & 25.0 & 0.008 & 1.047 \\
\hline Lao, People's Dem. Republic & 14.4 & 0.008 & 23.0 & 0.007 & 1.000 \\
\hline Mongolia & 65.1 & 0.038 & 222.0 & 0.069 & 1.000 \\
\hline Swaziland & 33.5 & 0.019 & 39.5 & 0.012 & 1.038 \\
\hline Albania & 76.5 & 0.044 & 144.5 & 0.045 & 1.000 \\
\hline Lesotho & 35.1 & 0.020 & 34.0 & 0.011 & 1.027 \\
\hline Equatorial Guinea & 3.4 & 0.002 & 8.0 & 0.003 & 1.000 \\
\hline Gambia, The & 13.5 & 0.008 & 15.4 & 0.005 & 1.054 \\
\hline Belize & 24.1 & 0.014 & 26.5 & 0.008 & 1.028 \\
\hline San Marino & 31.6 & 0.018 & 39.4 & 0.012 & 1.000 \\
\hline Vanuatu & 8.4 & 0.005 & 45.2 & 0.014 & 1.045 \\
\hline Djibouti & 14.0 & 0.008 & 19.6 & 0.006 & 1.000 \\
\hline Eritrea & 16.3 & 0.009 & 18.4 & 0.006 & 1.000 \\
\hline St. Lucia & 7.3 & 0.004 & 9.1 & 0.003 & 1.022 \\
\hline Guinea-Bissau & 6.4 & 0.004 & 15.7 & 0.005 & 1.000 \\
\hline Antigua and Barbuda & 10.8 & 0.006 & 23.2 & 0.007 & 1.020 \\
\hline Grenada & 8.3 & 0.005 & 14.0 & 0.004 & 1.037 \\
\hline Samoa & 4.3 & 0.003 & 3.7 & 0.001 & 1.026 \\
\hline Solomon Islands & 10.1 & 0.006 & 8.9 & 0.003 & 1.044 \\
\hline Cape Verde & 4.6 & 0.003 & 7.3 & 0.002 & 1.030 \\
\hline Comoros & 4.1 & 0.002 & 5.1 & 0.002 & $\mathbf{1} .000$ \\
\hline St. Kitts and Nevis & 2.6 & 0.002 & 8.0 & 0.003 & 1.021 \\
\hline Seychelles & 7.5 & 0.004 & 9.8 & 0.003 & 1.015 \\
\hline St. Vincent and the Grenadines & 7.3 & 0.004 & 4.7 & 0.001 & 1.018 \\
\hline Dominica & 2.5 & 0.001 & 3.5 & 0.001 & 1.028 \\
\hline
\end{tabular}




\begin{tabular}{|c|c|c|c|c|c|c|}
\hline \multirow{3}{*}{ Regression Variable Name } & & \multicolumn{2}{|c|}{$\begin{array}{c}\text { Variability of } \\
\text { Current Receipts } \\
\text { (1982-94) }\end{array}$} & \multicolumn{2}{|c|}{$\begin{array}{c}\text { Variability of } \\
\text { External Receipts } \\
(1982-94) \\
\end{array}$} & \multirow{2}{*}{$\begin{array}{l}\text { Variability of } \\
\text { Real Effective } \\
\text { Exchange Rates } \\
\text { (1982-94) } \\
\text { (Index) }\end{array}$} \\
\hline & - & $\begin{array}{l}\text { In millions of } \\
\text { SDRs }\end{array}$ & $\begin{array}{c}\text { In percent of } \\
\text { total }\end{array}$ & $\begin{array}{l}\text { In millions of } \\
\text { SDRs }\end{array}$ & $\begin{array}{c}\text { In percent of } \\
\text { total }\end{array}$ & \\
\hline & & \multicolumn{2}{|c|}{$\mathrm{VC}$} & \multicolumn{2}{|c|}{ VCK } & VREC \\
\hline Maldives & & 5.6 & 0.003 & 9.0 & 0.003 & 1.000 \\
\hline Sao Tome and Principe & & 1.4 & 0.001 & 2.9 & 0.001 & 1.000 \\
\hline Tonga & & 4.7 & 0.003 & 3.3 & 0.001 & 1.031 \\
\hline Bhutan & & 5.3 & 0.003 & 7.0 & 0.002 & 1.000 \\
\hline Kiribati & & 2.2 & 0.001 & 2.0 & 0.001 & 1.065 \\
\hline Micronesia, Fed. States of & & 2.6 & 0.001 & 3.3 & 0.001 & 1.000 \\
\hline Marshall Islands & & 3.1 & 0.002 & 3.6 & 0.001 & 1.000 \\
\hline Palau, Republic of & & 3.0 & 0.002 & 3.6 & 0.001 & 1.000 \\
\hline Total & & $173,231.4$ & 100.0 & $321,688.9$ & 100.0 & \\
\hline \multicolumn{7}{|l|}{ By WEO Classification } \\
\hline Advanced Economies & & $105,287.6$ & 60.8 & $236,012.2$ & 73.4 & \\
\hline Major Industrial Countries & & $76,921.4$ & 44.4 & $160,137.3$ & 49.8 & \\
\hline Other Advanced Economies & & $28,366.2$ & 16.4 & $75,875.0$ & 23.6 & \\
\hline Developing Countries & & $48,024.1$ & 27.7 & $62,592.1$ & 19.5 & \\
\hline Net Creditors & & $11,271.0$ & 6.5 & $11,234.6$ & 3.5 & \\
\hline Net Debtors & & $36,753.2$ & 21.2 & $51,357.6$ & 16.0 & \\
\hline $\begin{array}{l}\text { of which HIPC or least } \\
\text { developed countries }\end{array}$ & $\because$ & $3,272.1$ & 1.9 & $4,796.0$ & 1.5 & \\
\hline Transition Economies & & $19,919.6$ & 11.5 & $23,084.5$ & 7.2 & \\
\hline Total & & $173,231.4$ & 100.0 & $321,688.9$ & 100.0 & \\
\hline
\end{tabular}

1/ Defined as one standard deviation of current receipts from its five-year moving average centered on the third year, for the period 1982-94.

2/ Variability of current receipts plus capital and financial account credit; defined as one standard deviation from the five-year moving average centered on the third year, for the period 1982-94.

3/ Source: IMF Information Notice System (1982-94). Defined in terms of the deviation of the real effective exchange rate from a normal level, represented by a five-year moving average centered on the middle year. It is calculated as one standard deviation of the data from the normal level thus defined. The index shown is equal to $1+V / A$ where V=variability and $A=$ average value of the real effective exchange rate over 1982-94. The index is 1.0 for countries that do not have real effective exchange rate data.

4/ The variability of capital account receipts for each of the former Soviet Union Republics is calculated as a share of the total variability for the group of the 15 republics, with weights being the ratio of each republic's capital and financial account. 5/ Simple standard deviation of estimated capital account receipts based on the average ratio of capital and financial account debit to current account payments for the group of net debtor countries with diversified financing.

6/ Simple standard deviation of estimated capital account receipts based on the average ratio of capital and financial account debit to current account payments for the group of net debtor countries with official financing.

$7 /$ The average covariance between current and capital account receipts for the countries with available data in the Net Debtor developing country group with diversified financing is applied to the formula for the variance of current and capital account receipts to obtain the variability of external receipts.

8/ The average covariance between current and capital account receipts for the countries with available data in the Net Debtor developing country group with official financing is applied to the formula for the variance of current and capital account receipts to obtain the variability of external receipts. 
Table 1.7. Capital Flows and Debt

\begin{tabular}{|c|c|c|c|c|c|c|}
\hline \multirow[b]{3}{*}{ Regression Variable Name } & \multicolumn{2}{|c|}{$\begin{array}{l}\text { Normal Net Capital } \\
\text { Flows including } \\
\text { Errors and Omissions }{ }^{\text {If }} \\
\text { (1991-94) }\end{array}$} & \multicolumn{2}{|c|}{$\begin{array}{c}\text { Total External Debt }{ }^{2 /} \\
(1994)\end{array}$} & \multicolumn{2}{|c|}{$\begin{array}{c}\text { Short Term Debt }{ }^{3 /} \\
\text { (End 1994) }\end{array}$} \\
\hline & $\begin{array}{c}\text { In millions of } \\
\text { SDRs }\end{array}$ & $\begin{array}{c}\text { In percent of } \\
\text { total }\end{array}$ & $\begin{array}{l}\text { In millions of } \\
\text { SDRs }\end{array}$ & $\begin{array}{c}\text { In percent of } \\
\text { total }\end{array}$ & $\begin{array}{c}\text { In millions of } \\
\text { SDRs }\end{array}$ & $\begin{array}{c}\text { In percent of } \\
\text { total }\end{array}$ \\
\hline & \multicolumn{2}{|c|}{$\mathrm{NCF}$} & \multicolumn{2}{|c|}{ DEBT } & \multicolumn{2}{|c|}{ STDEBT } \\
\hline United States & $114,202.1$ & 30.566 & - & - & $8,887.2$ & 1.776 \\
\hline Japan & $-73,783.0$ & -19.748 & - & - & 32.2 & 0.006 \\
\hline Germany & $44,103.4$ & 11.804 & - & - & $2,805.8$ & 0.561 \\
\hline France & 986.8 & 0.264 & - & - & $2,664.0$ & 0.532 \\
\hline United Kingdom & $18,753.4$ & 5.019 & - & - & $15,458.4$ & 3.088 \\
\hline Italy & $19,993.5$ & 5.351 & - & - & $1,653.6$ & 0.330 \\
\hline Saudi Arabia & $12,312.4$ & 3.295 & - & - & $7,513.1$ & 1.501 \\
\hline Canada & $26,400.0$ & 7.066 & - & - & 469.2 & 0.094 \\
\hline Russia & 362.3 & 0.097 & $84,848.5$ & 6.475 & $12,888.3$ & 2.575 \\
\hline Netherlands & $-5,782.5$ & -1.548 & - & - & $4,478.5$ & 0.895 \\
\hline China & $5,868.9$ & 1.571 & $70,166.2$ & 5.355 & $15,940.0$ & 3.185 \\
\hline Belgium & $42,882.2$ & 11.477 & - & - & 614.4 & 0.123 \\
\hline India & $2,439.0$ & 0.653 & $71,581.1$ & 5.463 & $5,790.3$ & 1.157 \\
\hline Switzerland & $-15,425.5$ & -4.129 & - & - & 48.6 & 0.010 \\
\hline Australia & $8,814.1$ & 2.359 & - & - & $28,535.1$ & 5.701 \\
\hline Spain & $20,418.6$ & 5.465 & - & - & 7.5 & 0.002 \\
\hline Brazil & $4,938.4$ & 1.322 & $105,615.1$ & 8.060 & $20,781.6$ & 4.152 \\
\hline Venezuela & 681.4 & 0.182 & $25,740.4$ & 1.964 & $3,402.4$ & 0.680 \\
\hline Mexico & $22,270.7$ & 5.961 & $97,787.2$ & 7.463 & $28,312.5$ & 5.656 \\
\hline Sweden & $14,852.6$ & 3.975 & - & - & $6,306.8$ & 1.260 \\
\hline Argentina & $9,121.3$ & 2.441 & $56,113.4$ & 4.282 & $15,309.8$ & 3.059 \\
\hline Indonesia & $3,434.2$ & 0.919 & $75,311.8$ & 5.747 & $16,219.5$ & 3.240 \\
\hline Austria & $1,932.3$ & 0.517 & - & - & 780.9 & 0.156 \\
\hline South Africa & -18.9 & -0.005 & $15,136.6$ & 1.155 & $6,806.2$ & 1.360 \\
\hline Nigeria & $-1,605.7$ & -0.430 & $23,114.0$ & 1.764 & $1,220.0$ & 0.244 \\
\hline Norway & $-1,151.4$ & -0.308 & - & - & 28.8 & 0.006 \\
\hline Denmark & 246.1 & 0.066 & - & - & 575.4 & 0.115 \\
\hline Korea & $3,469.2$ & 0.929 & $65,683.0$ & 5.013 & $30,197.6$ & 6.033 \\
\hline Iran & $5,167.2$ & 1.383 & $15,808.0$ & 1.206 & $3,995.6$ & 0.798 \\
\hline Malaysia & $4,576.9$ & 1.225 & $21,188.7$ & 1.617 & $5,157.4$ & 1.030 \\
\hline Kuwait & $4,840.0$ & 1.295 & - & - & $3,758.6$ & 0.751 \\
\hline Ukraine & 749.9 & 0.201 & $3,909.2$ & 0.298 & 198.0 & 0.040 \\
\hline Poland & $3,668.4$ & 0.982 & $29,721.9$ & 2.268 & $1,628.2$ & 0.325 \\
\hline Finiand & $7,793.9$ & 2.086 & - & - & $1,165.9$ & 0.233 \\
\hline Algeria & $1,148.3$ & 0.307 & $20,935.4$ & 1.598 & $4,738.8$ & 0.947 \\
\hline
\end{tabular}


Table 1.7. Capital Flows and Debt

\begin{tabular}{|c|c|c|c|c|c|c|}
\hline \multirow[b]{3}{*}{ Regression Variable Name } & \multicolumn{2}{|c|}{$\begin{array}{c}\text { Normal Net Capital } \\
\text { Flows including } \\
\text { Errors and Omissions }{ }^{1 /} \\
(1991-94)\end{array}$} & \multicolumn{2}{|c|}{$\begin{array}{c}\text { Total External Debt }{ }^{2 /} \\
\text { (1994) }\end{array}$} & \multicolumn{2}{|c|}{$\begin{array}{c}\text { Short Term Debt }{ }^{3 /} \\
\text { (End 1994) }\end{array}$} \\
\hline & $\begin{array}{l}\text { In millions of } \\
\text { SDRs }\end{array}$ & $\begin{array}{c}\text { In percent of } \\
\text { total }\end{array}$ & $\begin{array}{l}\text { In millions of } \\
\quad \text { SDRs }\end{array}$ & $\begin{array}{l}\text { In percent of } \\
\text { total }\end{array}$ & $\begin{array}{l}\text { In millions of } \\
\text { SDRs }\end{array}$ & $\begin{array}{c}\text { In percent of } \\
\text { total }\end{array}$ \\
\hline & \multicolumn{2}{|c|}{$\mathrm{NCF}$} & \multicolumn{2}{|c|}{ DEBT } & \multicolumn{2}{|c|}{ STDEBT } \\
\hline & & & & & & \\
\hline Iraq & 409.2 & 0.110 & - & - & $1,641.9$ & 0.328 \\
\hline Libya & $1,038.0$ & 0.278 & - & - & 142.5 & 0.028 \\
\hline Thailand & $7,571.0$ & 2.026 & $45,817.1$ & 3.497 & $22,254.3$ & 4.446 \\
\hline Hungary & $2,747.4$ & 0.735 & $19,672.8$ & 1.501 & $2,438.6$ & 0.487 \\
\hline Pakistan & $2,633.7$ & 0.705 & $19,109.5$ & 1.458 & $1,665.2$ & 0.333 \\
\hline Romania & $1,048.2$ & 0.281 & $3,864.3$ & 0.295 & 559.0 & 0.112 \\
\hline Turkey & $1,730.1$ & 0.463 & $46,277.3$ & 3.532 & $6,976.7$ & 1.394 \\
\hline Egypt & $1,456.9$ & 0.390 & $22,570.0$ & 1.722 & $1,805.0$ & 0.361 \\
\hline Israel & $2,421.7$ & 0.648 & - & - & $1,859.8$ & 0.372 \\
\hline New Zealand & 650.7 & 0.174 & - & - & $3,584.6$ & 0.716 \\
\hline Philippines & $3,081.7$ & 0.825 & $27,527.8$ & 2.101 & $2,832.5$ & 0.566 \\
\hline Portugal & 337.5 & 0.090 & - & - & $10,555.9$ & 2.109 \\
\hline Singapore & $2,107.9$ & 0.564 & - & - & $114,222.7$ & 22.820 \\
\hline Chile & $1,987.5$ & 0.532 & $17,272.1$ & 1.318 & $4,860.1$ & 0.971 \\
\hline Ireland & -251.5 & -0.067 & - & - & 122.6 & 0.024 \\
\hline Greece & $3,926.5$ & 1.051 & - & - & $7,275.4$ & 1.454 \\
\hline Czech Republic & $1,412.0$ & 0.378 & $7,469.3$ & 0.570 & $1,278.9$ & 0.256 \\
\hline Colombia & 704.5 & 0.189 & $15,324.2$ & 1.169 & $3,779.8$ & 0.755 \\
\hline Bulgaria & 744.9 & 0.199 & $6,845.8$ & 0.522 & 529.5 & 0.106 \\
\hline Peru & $2,716.3$ & 0.727 & $18,527.1$ & 1.414 & $1,691.3$ & 0.338 \\
\hline United Arab Emirates & $1,363.5$ & 0.365 & - & - & $2,422.2$ & 0.484 \\
\hline Morocco & 831.2 & 0.222 & $15,476.6$ & 1.181 & $1,404.3$ & 0.281 \\
\hline Bangladesh & 714.1 & 0.191 & $11,355.7$ & 0.867 & 604.2 & 0.121 \\
\hline Congo, Dem. Republic of & 141.8 & 0.038 & $8,606.7$ & 0.657 & 145.2 & 0.029 \\
\hline Zambia & 149.4 & 0.040 & $4,598.2$ & 0.351 & 111.0 & 0.022 \\
\hline FRY (Serbia/Montenegro) & -174.9 & -0.047 & - & - & $1,152.1^{4}$ & 0.230 \\
\hline Sri Lanka & 940.5 & 0.252 & $5,509.4$ & 0.420 & 452.1 & 0.090 \\
\hline Belarus & 165.4 & 0.044 & 888.8 & 0.068 & 41.8 & 0.008 \\
\hline Ghana & 316.4 & 0.085 & $3,813.0$ & 0.291 & 68.5 & 0.014 \\
\hline Kazakhstan & 88.9 & 0.024 & $1,948.5$ & 0.149 & 104.1 & 0.021 \\
\hline Croatia & 99.8 & 0.027 & $1,434.8$ & 0.109 & 154.1 & 0.031 \\
\hline Slovak Republic & 319.0 & 0.085 & $3,240.4$ & 0.247 & 289.1 & 0.058 \\
\hline Zimbabwe & 522.2 & 0.140 & $3,168.7$ & 0.242 & 475.4 & 0.095 \\
\hline Trinidad and Tobago & -130.3 & -0.035 & $1,742.5$ & 0.133 & 317.8 & 0.063 \\
\hline Vietnam & 408.7 & 0.109 & $17,322.2$ & 1.322 & 326.1 & 0.065 \\
\hline
\end{tabular}




\begin{tabular}{|c|c|c|c|c|c|c|}
\hline \multirow[b]{3}{*}{ Regression Variable Name } & \multicolumn{2}{|c|}{$\begin{array}{c}\text { Normal Net Capital } \\
\text { Flows including } \\
\text { Errors and Omissions }{ }^{1 /} \\
(1991-94)\end{array}$} & \multicolumn{2}{|c|}{$\begin{array}{c}\text { Total Extemal Debt }{ }^{2 /} \\
(1994)\end{array}$} & \multicolumn{2}{|c|}{$\begin{array}{l}\text { Short Term Debt }{ }^{3 /} \\
\text { (End 1994) }\end{array}$} \\
\hline & $\begin{array}{l}\text { In millions of } \\
\text { SDRs }\end{array}$ & $\begin{array}{c}\text { In percent of } \\
\text { total }\end{array}$ & $\begin{array}{l}\text { In millions of } \\
\text { SDRs }\end{array}$ & $\begin{array}{c}\text { In percent of } \\
\text { total }\end{array}$ & $\begin{array}{l}\text { In millions of } \\
\text { SDRs }\end{array}$ & $\begin{array}{l}\text { In percent of } \\
\text { total }\end{array}$ \\
\hline & \multicolumn{2}{|c|}{$\mathrm{NCF}$} & \multicolumn{2}{|c|}{ DEBT } & \multicolumn{2}{|c|}{ STDEBT } \\
\hline Cote dTvoire & $1,222.5$ & 0.327 & $12,150.0$ & 0.927 & $1,267.9$ & 0.253 \\
\hline Sudan & 821.1 & 0.220 & $11,816.7$ & 0.902 & 404.8 & 0.081 \\
\hline Uruguay & 298.7 & 0.080 & $3,546.3$ & 0.271 & $1,694.0$ & 0.338 \\
\hline Ecuador & 142.6 & 0.038 & $10,529.5$ & 0.804 & $1,437.1$ & 0.287 \\
\hline Syrian Arab Republic & 304.0 & 0.081 & $14,359.2$ & 1.096 & $2,752.2^{4}$ & 0.550 \\
\hline Tunisia & 988.6 & 0.265 & $6,711.9$ & 0.512 & 594.6 & 0.119 \\
\hline Angola & 759.1 & 0.203 & $7,890.0$ & 0.602 & 696.0 & 0.139 \\
\hline Luxembourg & 310.8 & 0.083 & - & - & $2,381.1$ & 0.476 \\
\hline Uzbekistan & 74.4 & 0.020 & 868.8 & 0.066 & 130.8 & 0.026 \\
\hline Jamaica & 129.6 & 0.035 & $3,013.7$ & 0.230 & 287.7 & 0.057 \\
\hline Kenya & 236.9 & 0.063 & $5,006.8$ & 0.382 & 172.6 & 0.034 \\
\hline Qatar & 339.5 & 0.091 & - & - & 887.8 & 0.177 \\
\hline Myanmar & 195.0 & 0.052 & $4,578.6$ & 0.349 & 28.1 & 0.006 \\
\hline Yemen, kepublic of & 361.5 & 0.097 & $4,278.2$ & 0.326 & 173.3 & 0.035 \\
\hline Slovenia & -97.6 & -0.026 & - & - & 351.4 & $\begin{array}{c}0.070 \\
-\end{array}$ \\
\hline Dominican Republic & 242.6 & 0.065 & $2,985.9$ & 0.228 & 238.4 & 0.048 \\
\hline Brunei Darussalam & 220.2 & 0.059 & - & - & 180.2 & 0.036 \\
\hline Guatemala & 446.5 & 0.119 & $2,396.0$ & 0.183 & 345.2 & 0.069 \\
\hline Panama & $1,122.0$ & 0.300 & $4,979.3$ & 0.380 & $2,099.7^{4 /}$ & 0.419 \\
\hline Lebanon & 62.5 & 0.017 & $1,479.5$ & 0.113 & $917.9^{4}$ & 0.183 \\
\hline Tanzania & $1,023.6$ & 0.274 & $5,077.7$ & 0.388 & 71.2 & 0.014 \\
\hline Oman & 405.2 & 0.108 & $2,156.2$ & 0.165 & 652.1 & 0.130 \\
\hline Cameroon & 746.2 & 0.200 & $5,815.5$ & 0.444 & 476.8 & 0.095 \\
\hline Uganda & 300.6 & 0.080 & $2,355.0$ & 0.180 & 34.3 & 0.007 \\
\hline Bolivia & 374.2 & 0.100 & $3,406.2$ & 0.260 & 204.8 & 0.041 \\
\hline El Salvador & 124.3 & 0.033 & $1,543.5$ & 0.118 & 132.2 & 0.026 \\
\hline Jordan & 760.1 & 0.203 & $5,384.0$ & 0.411 & 545.3 & 0.109 \\
\hline Bosnia-Herzegovina & 5.2 & 0.001 & - & - & $26.4^{4 t}$ & 0.005 \\
\hline Costa Rica & 267.5 & 0.072 & $2,728.2$ & 0.208 & $1,767.3$ & 0.353 \\
\hline Afghanistan, Islamic State of & 13.5 & 0.004 & - & - & 6.2 & 0.001 \\
\hline Senegal & 595.6 & 0.159 & $2,555.1$ & 0.195 & 162.3 & 0.032 \\
\hline Azerbaijan & 23.2 & 0.006 & 78.8 & 0.006 & 4.8 & 0.001 \\
\hline Gabon & 86.5 & 0.023 & $2,913.5$ & 0.222 & 103.4 & 0.021 \\
\hline Georgia & 0.6 & 0.000 & 985.3 & 0.075 & 327.4 & 0.065 \\
\hline Lithuania & 108.4 & 0.029 & 344.8 & 0.026 & 26.7 & 0.005 \\
\hline
\end{tabular}


Table L.7. Capital Flows and Debt

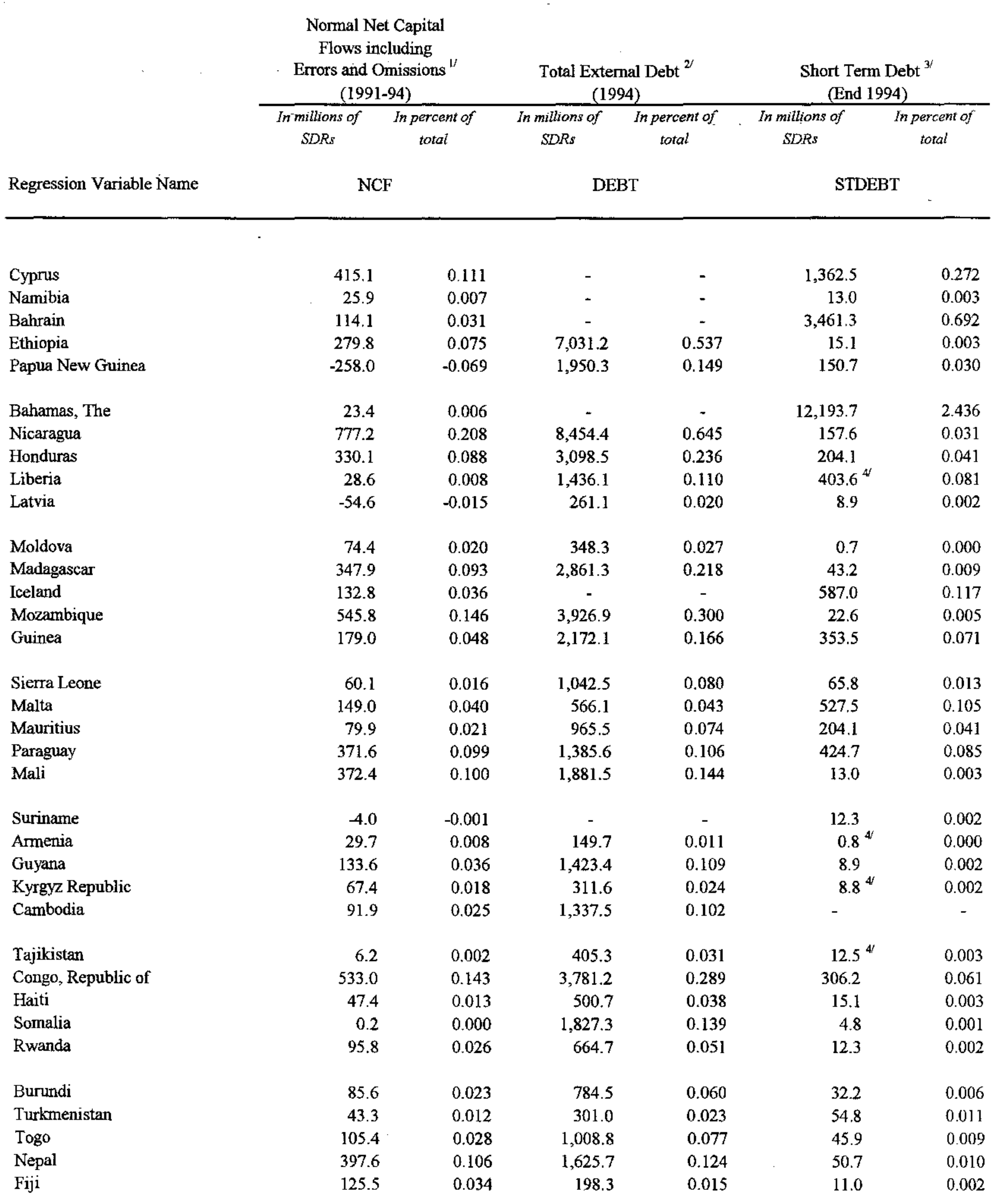


Table I.7. Capital Flows and Debt

\begin{tabular}{|c|c|c|c|c|c|c|}
\hline \multirow[b]{3}{*}{ Regression Variable Name } & \multicolumn{2}{|c|}{$\begin{array}{l}\text { Normal Net Capital } \\
\text { Flows including } \\
\text { Errors and Omissions }{ }^{1 /} \\
(1991-94)\end{array}$} & \multicolumn{2}{|c|}{$\begin{array}{c}\text { Total External Debt }{ }^{2 /} \\
\text { (1994) }\end{array}$} & \multicolumn{2}{|c|}{$\begin{array}{c}\text { Short Term Debt } \\
\text { (End 1994) }\end{array}$} \\
\hline & $\begin{array}{l}\text { In-millions of } \\
\quad \text { SDRs }\end{array}$ & $\begin{array}{c}\text { In percent of } \\
\text { total }\end{array}$ & $\begin{array}{l}\text { In millions of } \\
\text { SDRs }\end{array}$ & $\begin{array}{l}\text { In percent of } \\
\text { total }\end{array}$ & $\begin{array}{l}\text { In millions of } \\
\text { SDRs }\end{array}$ & $\begin{array}{c}\text { In percent of } \\
\text { total }\end{array}$ \\
\hline & \multicolumn{2}{|c|}{ NCF } & \multicolumn{2}{|c|}{ DEBT } & \multicolumn{2}{|c|}{ STDEBT } \\
\hline Malawi & 255.3 & 0.068 & $1,414.3$ & 0.108 & 24.0 & 0.005 \\
\hline Macedonia, FYR & 27.2 & 0.007 & 770.8 & 0.059 & $107.1^{4 /}$ & 0.021 \\
\hline Barbados & -46.8 & -0.013 & 429.3 & 0.033 & $130.4^{4}$ & 0.026 \\
\hline Niger & 167.7 & 0.045 & $1,065.4$ & 0.081 & 16.4 & 0.003 \\
\hline Estonia & 53.5 & 0.014 & 129.9 & 0.010 & 9.6 & 0.002 \\
\hline Mauritania & 79.7 & 0.021 & $1,552.9$ & 0.119 & 54.1 & 0.011 \\
\hline Botswana & 78.1 & 0.021 & 481.0 & 0.037 & 108.2 & 0.022 \\
\hline Benin & 125.6 & 0.034 & $1,110.1$ & 0.085 & 20.6 & 0.004 \\
\hline Burkina Faso & 131.6 & 0.035 & 788.3 & 0.060 & 24.7 & 0.005 \\
\hline Chad & 105.7 & 0.028 & 578.4 & 0.044 & 5.5 & 0.001 \\
\hline Central African Republic & 103.5 & 0.028 & 620.2 & 0.047 & 15.1 & 0.003 \\
\hline Lao, People's Dem. Republic & 93.4 & 0.025 & $1,452.9$ & 0.111 & 7.5 & 0.002 \\
\hline Mongolia & 18.3 & 0.005 & 312.4 & 0.024 & 8.9 & 0.002 \\
\hline Swaziland & -14.4 & -0.004 & 153.4 & 0.012 & 21.2 & 0.004 \\
\hline Albania & 145.3 & 0.039 & 594.5 & 0.045 & 256.9 & 0.051 \\
\hline Lesotho & 5.0 & 0.001 & 432.7 & 0.033 & 428.1 & 0.086 \\
\hline Equatorial Guinea & 16.9 & 0.005 & 200.9 & 0.015 & 7.5 & 0.002 \\
\hline Gambia, The & 20.1 & 0.005 & 296.6 & 0.023 & 245.9 & 0.049 \\
\hline Belize & 26.7 & 0.007 & 140.2 & 0.011 & 15.1 & 0.003 \\
\hline San Marino & 9.2 & 0.002 & - & - & - & - \\
\hline Vanuatu & 31.0 & 0.008 & 32.5 & 0.002 & $3.4^{4}$ & 0.001 \\
\hline Djibouti & 40.0 & 0.011 & 183.8 & 0.014 & 7.5 & 0.002 \\
\hline Eritrea & 18.6 & 0.005 & 20.3 & 0.002 & - & - \\
\hline St. Lucia & 53.0 & 0.014 & 78.2 & 0.006 & 7.5 & 0.002 \\
\hline Guinea-Bissau & 107.1 & 0.029 & 595.2 & 0.045 & 34.3 & 0.007 \\
\hline Antigua and Barbuda & 16.2 & 0.004 & - & - & 2.7 & 0.001 \\
\hline Grenada & 37.8 & 0.010 & 80.7 & 0.006 & 6.9 & 0.001 \\
\hline Samoa & 30.1 & 0.008 & 109.6 & 0.008 & - & $\sim$ \\
\hline Solomon Islands & 3.5 & 0.001 & 108.2 & 0.008 & 1.4 & 0.000 \\
\hline Cape Verde & 33.7 & 0.009 & 125.5 & 0.010 & 4.1 & 0.001 \\
\hline Comoros & 18.5 & 0.005 & 134.3 & 0.010 & 0.7 & 0.000 \\
\hline St. Kitts and Nevis & 24.7 & 0.007 & 40.6 & 0.003 & 2.1 & 0.000 \\
\hline Seychelles & 32.6 & 0.009 & 119.4 & 0.009 & 15.8 & 0.003 \\
\hline St. Vincent and the Grenadines & 40.9 & 0.011 & 98.4 & 0.008 & 42.5 & 0.008 \\
\hline Dominica & 29.7 & 0.008 & 67.8 & 0.005 & 4.8 & 0.001 \\
\hline
\end{tabular}


Table 1.7. Capital Flows and Debt

\begin{tabular}{|c|c|c|c|c|c|c|}
\hline \multirow[b]{3}{*}{ Regression Variable Name } & \multicolumn{2}{|c|}{$\begin{array}{c}\text { Normal Net Capital } \\
\text { Flows including } \\
\text { Errors and Omissions }{ }^{1 /} \\
(1991-94) \\
\end{array}$} & \multicolumn{2}{|c|}{$\begin{array}{c}\text { Total External Debt }{ }^{2 /} \\
(1994)\end{array}$} & \multicolumn{2}{|c|}{$\begin{array}{c}\text { Short Term Debt }{ }^{3 /} \\
\text { (End 1994) }\end{array}$} \\
\hline & $\begin{array}{l}\text { In-millions of } \\
\quad \text { SDRs }\end{array}$ & $\begin{array}{c}\text { In percent of } \\
\text { total }\end{array}$ & $\begin{array}{l}\text { In millions of } \\
\text { SDRs }\end{array}$ & $\begin{array}{c}\text { In percent of } \\
\text { total }\end{array}$ & $\begin{array}{l}\text { In millions of } \\
\text { SDRs }\end{array}$ & $\begin{array}{c}\text { In percent of } \\
\text { total }\end{array}$ \\
\hline & \multicolumn{2}{|c|}{ NCF } & \multicolumn{2}{|c|}{ DEBT } & \multicolumn{2}{|c|}{ STDEBT } \\
\hline Maldives & 26.9 & 0.007 & 86.3 & 0.007 & 8.9 & 0.002 \\
\hline Sao Tome and Principe & 0.5 & 0.000 & 159.4 & 0.012 & 4.1 & 0.001 \\
\hline Tonga & 2.3 & 0.001 & 45.0 & 0.003 & 0.7 & 0.000 \\
\hline Bhutan & 8.6 & 0.002 & 61.0 & 0.005 & 1.4 & 0.000 \\
\hline Kiribati & -2.3 & -0.001 & - & $\cdot$ & 1.4 & 0.000 \\
\hline Micronesia, Fed. States of & 6.7 & 0.002 & - & - & - & - \\
\hline Marshall Islands & 4.2 & 0.001 & - & - & - & - \\
\hline Palau, Republic of & 3.7 & 0.001 & - & - & - & - \\
\hline Total & $373,628.0$ & 100.0 & $1,310,348.4$ & 100.0 & $500,542.2$ & 100.0 \\
\hline By WEO Classification & & & & & & \\
\hline Advanced Economies & $238,351.4$ & 63.8 & $65,683.0$ & 5.0 & $245,299.2$ & 49.0 \\
\hline Major Industrial Countries & $150,656.2$ & 40.3 & - & - & $31,970.4$ & 6.4 \\
\hline Other Advanced Economies & $87,695.2$ & 23.5 & $65,683.0$ & 5.0 & $213,328.8$ & 42.6 \\
\hline Developing Countries & $123,520.2$ & 33.1 & $1,074,959.0$ & 82.0 & $232,644.9$ & 46.5 \\
\hline Net Creditors & $20,518.9$ & 5.5 & $2,156.2$ & 0.2 & $15,556.4$ & 3.1 \\
\hline $\begin{array}{l}\text { Net Debtors } \\
\text { of which HIPC or least }\end{array}$ & $103,001.4$ & 27.6 & $1,072,802.8$ & 81.9 & $217,088.5$ & 43.4 \\
\hline developed countries & $14,271.6$ & 3.8 & $167,073.3$ & 12.8 & $7,732.4$ & 1.5 \\
\hline Transition Economies & $11,756.3$ & 3.1 & $169,706.4$ & 13.0 & $22,598.1$ & 4.5 \\
\hline Total & $373,628.0$ & 100.0 & $1,310,348.4$ & 100.0 & $500,542.2$ & 100.0 \\
\hline
\end{tabular}

1/ Proxied by a four-year average of net private capital flows and including errors and omissions for the period 1991-94.

2/ Source: 1996 World Bank World Development Indicators; defined as debt owed to non-residents repayable in foreign currency, goods, and services. It is the sum of public, publicly guaranteed, and private non-guaranteed debt, use of IMF credit, and short-term debt. 3/ Sources: For Industrial Countries, the Bank of International Settlement database; for Developing countries, the Joint BIS-IMF-OECDWorld Bank Statistics on External Debt, except where noted.

4/ Source: World Bank Global Development Finance database. 
Table I.8. Qualitative Variables

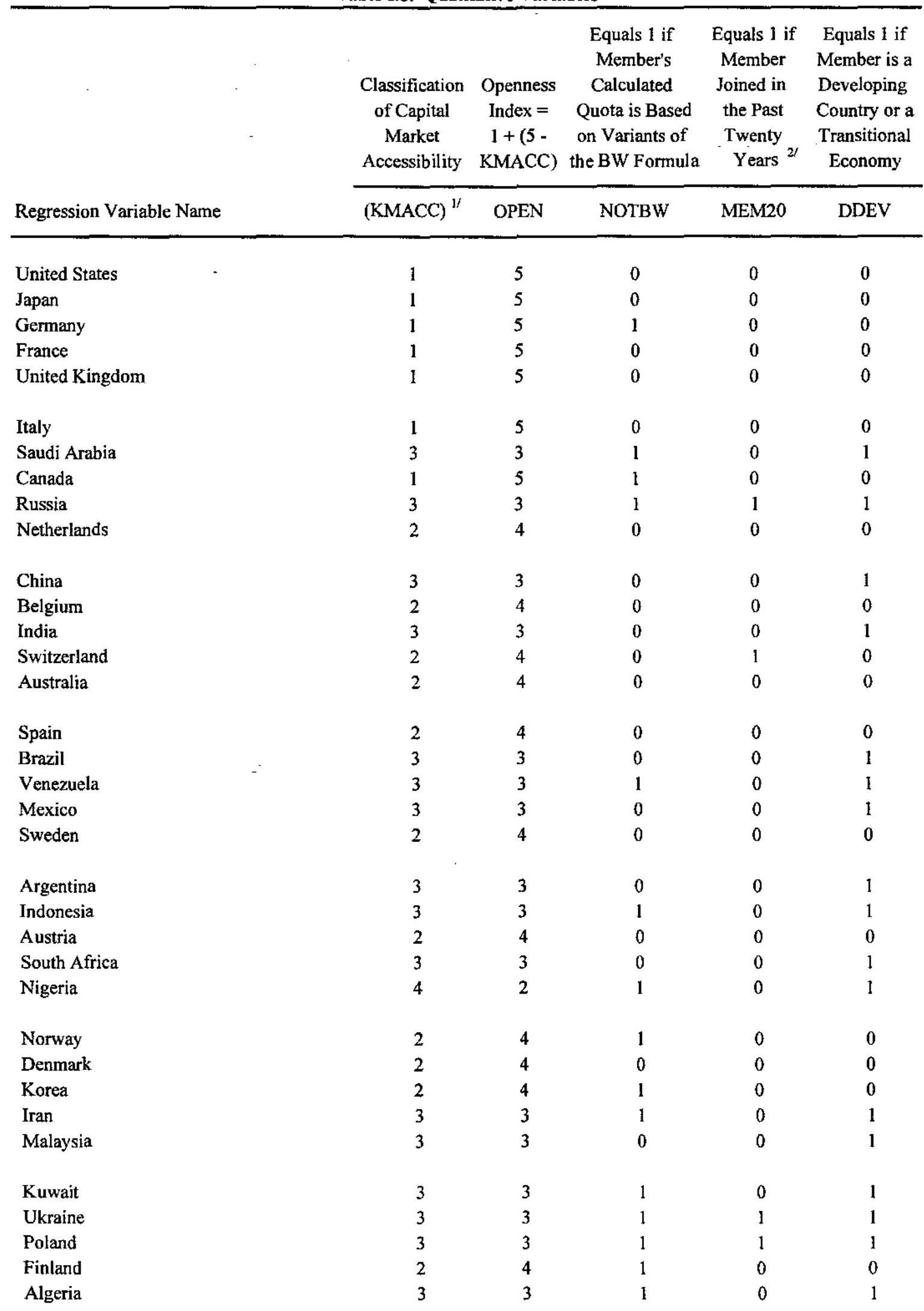


Table I.8. Qualitative Variables

\begin{tabular}{|c|c|c|c|c|c|}
\hline . & $\begin{array}{l}\text { Classification } \\
\text { of Capital } \\
\text { Market } \\
\text { Accessibility }\end{array}$ & $\begin{array}{c}\text { Openness } \\
\text { Index }= \\
1+(5- \\
\text { KMACC })\end{array}$ & $\begin{array}{c}\text { Equals } 1 \text { if } \\
\text { Member's } \\
\text { Calculated } \\
\text { Quota is Based } \\
\text { on Variants of } \\
\text { the BW Formula }\end{array}$ & $\begin{array}{l}\text { Equals } 1 \text { if } \\
\text { Member } \\
\text { Joined in } \\
\text { the Past } \\
\text { Twenty } \\
\text { Years }^{2}\end{array}$ & $\begin{array}{l}\text { Equals } 1 \text { if } \\
\text { Member is a } \\
\text { Developing } \\
\text { Country or a } \\
\text { Transitional } \\
\text { Economy }\end{array}$ \\
\hline Regression Variable Name & $(\mathrm{KMACC})^{1 /}$ & OPEN & NOTBW & MEM20 & DDEV \\
\hline Iraq & 3 & 3 & 1 & 0 & 1 \\
\hline Libya & 3 & 3 & 1 & 0 & 1 \\
\hline Thailand & 3 & 3 & 0 & 0 & 1 \\
\hline Hungary & 3 & 3 & 1 & 1 & 1 \\
\hline Pakistan & 4 & 2 & 1 & 0 & 1 \\
\hline Romania & 3 & 3 & 1 & 0 & 1 \\
\hline Turkey & 3 & 3 & 1 & 0 & 1 \\
\hline Egypt & 3 & 3 & 1 & 0 & 1 \\
\hline Israel & 2 & 4 & 0 & 0 & 0 \\
\hline New Zealand & 2 & 4 & 1 & 0 & 0 \\
\hline Philippines & 3 & 3 & 1 & 0 & 1 \\
\hline Portugal & 2 & 4 & 1 & 0 & 0 \\
\hline Singapore & 2 & 4 & 0 & 0 & 0 \\
\hline Chile & 3 & 3 & 1 & 0 & 1 \\
\hline Ireland & 2 & 4 & 0 & 0 & 0 \\
\hline Greece & 2 & 4 & 0 & 0 & 0 \\
\hline Czech Republic & 3 & 3 & 1 & 1 & 1 \\
\hline Colombia & 3 & 3 & 0 & 0 & 1 \\
\hline Bulgaria & 3 & 3 & 1 & 1 & 1 \\
\hline Peru & 3 & 3 & 0 & 0 & 1 \\
\hline United Arab Emirates & 3 & 3 & 1 & 0 & 1 \\
\hline Morocco & 4 & 2 & 1 & 0 & 1 \\
\hline Bangladesh & 4 & 2 & 0 & 0 & 1 \\
\hline Congo, Dem. Republic of & 4 & 2 & 1 & 0 & 1 \\
\hline Zambia & 4 & 2 & 1 & 0 & 1 \\
\hline FRY (Serbia/Montenegro) & 3 & 3 & 1 & 0 & 1 \\
\hline Sri Lanka & 3 & 3 & l & 0 & 1 \\
\hline Belarus & 3 & 3 & 1 & 1 & 1 \\
\hline Ghana & 3 & 3 & 0 & 0 & 1 \\
\hline Kazakstan & 3 & 3 & 1 & 1 & 1 \\
\hline Croatia & 3 & 3 & 1 & 1 & 1 \\
\hline Slovak Republic & 3 & 3 & 1 & 1 & 1 \\
\hline Zimbabwe & 4 & 2 & 1 & 1 & 1 \\
\hline Trinidad and Tobago & 3 & 3 & 1 & 0 & 1 \\
\hline Vietnam & 4 & 2 & 1 & 0 & 1 \\
\hline
\end{tabular}


Table I.8. Qualitative Variables

\begin{tabular}{|c|c|c|c|c|c|}
\hline 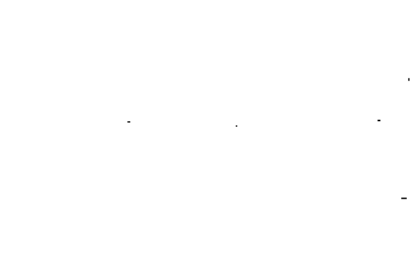 & $\begin{array}{l}\text { Classification } \\
\text { of Capital } \\
\text { Market } \\
\text { Accessibility }\end{array}$ & $\begin{array}{c}\text { Openness } \\
\text { Index }= \\
1+(5- \\
\text { KMACC })\end{array}$ & $\begin{array}{c}\text { Equals } 1 \text { if } \\
\text { Member's } \\
\text { Calculated } \\
\text { Quota is Based } \\
\text { on Variants of } \\
\text { the BW Formula }\end{array}$ & $\begin{array}{l}\text { Equals I if } \\
\text { Member } \\
\text { Joined in } \\
\text { the Past } \\
\text { Twenty } \\
\text { Years }^{2}\end{array}$ & $\begin{array}{l}\text { Equals } 1 \text { if } \\
\text { Member is a } \\
\text { Developing } \\
\text { Country or a } \\
\text { Transitional } \\
\text { Economy }\end{array}$ \\
\hline Regression Variable Name & $(\mathrm{KMACC})^{1 /}$ & OPEN & NOTBW & MEM20 & DDEV \\
\hline Cote d'Ivoire & 3 & 3 & 1 & 0 & 1 \\
\hline Sudan & 4 & 2 & 1 & 0 & 1 \\
\hline Uruguay & 3 & 3 & 0 & 0 & 1 \\
\hline Ecuador & 3 & 3 & 1 & 0 & 1 \\
\hline Syrian Arab Republic & 3 & 3 & 1 & 0 & 1 \\
\hline Tunisia & 3 & 3 & 0 & 0 & 1 \\
\hline Angola & 3 & 3 & 1 & 1 & 1 \\
\hline Luxembourg & 2 & 4 & 0 & 0 & 0 \\
\hline Uzbekistan & 3 & 3 & 1 & 1 & 1 \\
\hline Jamaica & 3 & 3 & 0 & 0 & 1 \\
\hline Kenya & 4 & 2 & 1 & 0 & 1 \\
\hline Qatar & 3 & 3 & 1 & 0 & 1 \\
\hline Myanmar & 3 & 3 & 0 & 0 & 1 \\
\hline Yemen, Republic of & 3 & 3 & 1 & 1 & 1 \\
\hline Slovenia & 3 & 3 & 1 & 1 & 1 \\
\hline Dominican Republic & 3 & 3 & 1 & 0 & 1 \\
\hline Brunei Darussalam & 3 & 3 & 1 & 1 & 1 \\
\hline Guatemala & 3 & 3 & 0 & 0 & 1 \\
\hline Panama & 3 & 3 & 1 & 0 & 1 \\
\hline Lebanon & 3 & 3 & 1 & 0 & 1 \\
\hline Tanzania & 4 & 2 & 1 & 0 & 1 \\
\hline Oman & 3 & 3 & 1 & 0 & 1 \\
\hline Cameroon & 4 & 2 & 1 & 0 & 1 \\
\hline Uganda & 4 & 2 & 0 & 0 & 1 \\
\hline Bolivia & 4 & 2 & 1 & 0 & 1 \\
\hline El Salvador & 4 & 2 & 1 & 0 & 1 \\
\hline Jordan & 4 & 2 & 0 & 0 & 1 \\
\hline Bosnia-Herzegovina & 3 & 3 & 0 & 1 & 1 \\
\hline Costa Rica & 3 & 3 & 1 & 0 & 1 \\
\hline Afghanistan, Islamic State of & 3 & 3 & 1 & 0 & 1 \\
\hline Senegal & 4 & 2 & 0 & 0 & 1 \\
\hline Azerbaijan & 3 & 3 & 1 & 1 & 1 \\
\hline Gabon & 4 & 2 & 1 & 0 & 1 \\
\hline Georgia & 3 & 3 & 1 & 1 & 1 \\
\hline Lithuania & 3 & 3 & 1 & 1 & 1 \\
\hline
\end{tabular}


Table I.8. Qualitative Variables

\begin{tabular}{|c|c|c|c|c|c|}
\hline 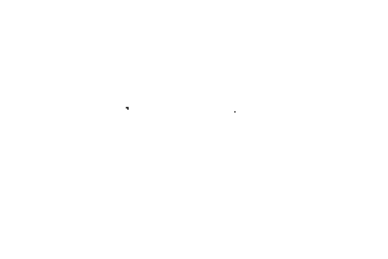 & $\begin{array}{c}\text { Classification } \\
\text { of Capital } \\
\text { Market } \\
\text { Accessibility }\end{array}$ & $\begin{array}{l}\text { Openness } \\
\text { Index }= \\
1+(5- \\
\text { KMACC })\end{array}$ & $\begin{array}{l}\text { Equals } 1 \text { if } \\
\text { Member's } \\
\text { Calculated } \\
\text { Quota is Based } \\
\text { on Variants of } \\
\text { the BW Formula }\end{array}$ & $\begin{array}{l}\text { Equals } 1 \text { if } \\
\text { Member } \\
\text { Joined in } \\
\text { the Past } \\
\text { Twenty } \\
\text { Years }\end{array}$ & $\begin{array}{l}\text { Equals } 1 \text { if } \\
\text { Member is a } \\
\text { Developing } \\
\text { Country or a } \\
\text { Transitional } \\
\text { Economy }\end{array}$ \\
\hline Regression Variable Name & $(\mathrm{KMACC})^{1 /}$ & OPEN & NOTBW & MEM20 & DDEV \\
\hline Cyprus & 3 & 3 & 1 & 0 & 1 \\
\hline Namibia & 4 & 2 & 0 & 1 & 1 \\
\hline Bahrain & 3 & 3 & 0 & 0 & 1 \\
\hline Ethiopia & 4 & 2 & 1 & 0 & 1 \\
\hline Papua New Guinea & 3 & 3 & 1 & 0 & 1 \\
\hline Bahamas, The & 3 & 3 & 1 & 0 & 1 \\
\hline Nicaragua & 4 & 2 & 1 & 0 & 1 \\
\hline Honduras & 4 & 2 & 1 & 0 & 1 \\
\hline Liberia & 4 & 2 & 1 & 0 & 1 \\
\hline Latvia & 3 & 3 & 1 & 1 & 1 \\
\hline Moldova & 3 & 3 & 1 & 1 & 1 \\
\hline Madagascar & 4 & 2 & 0 & 0 & 1 \\
\hline Iceland & 2 & 4 & 0 & 0 & 0 \\
\hline Mozambique & 4 & 2 & 0 & 1 & 1 \\
\hline Guinea & 3 & 3 & 1 & 0 & 1 \\
\hline Sierra Leone & 3 & 3 & 1 & 0 & 1 \\
\hline Malta & 3 & 3 & 0 & 0 & 1 \\
\hline Mauritius & 3 & 3 & 0 & 0 & 1 \\
\hline Paraguay & 4 & 2 & 1 & 0 & 1 \\
\hline Mali & 3 & 3 & 0 & 0 & 1 \\
\hline Suriname & 3 & 3 & 0 & 0 & 1 \\
\hline Armenia & 3 & 3 & 0 & 1 & 1 \\
\hline Guyana & 4 & 2 & 0 & 0 & 1 \\
\hline Kyrgyz Republic & 3 & 3 & 1 & 1 & 1 \\
\hline Cambodia & 4 & 2 & 0 & 0 & 1 \\
\hline Tajikistan & 3 & 3 & 0 & 1 & 1 \\
\hline Congo, Republic of & 4 & 2 & 1 & 0 & 1 \\
\hline Haiti & 4 & 2 & 1 & 0 & 1 \\
\hline Somalia & 4 & 2 & 0 & 0 & 1 \\
\hline Rwanda & 4 & 2 & 1 & 0 & 1 \\
\hline Burundi & 4 & 2 & 1 & 0 & 1 \\
\hline Turkmenistan & 3 & 3 & 1 & 1 & 1 \\
\hline Togo & 3 & 3 & 1 & 0 & 1 \\
\hline Nepal & 4 & 2 & 0 & 0 & 1 \\
\hline Fiji & 3 & 3 & 1 & 0 & 1 \\
\hline
\end{tabular}


Table I.8. Qualitative Variables

\begin{tabular}{|c|c|c|c|c|c|}
\hline , & $\begin{array}{l}\text { Classification } \\
\text { of Capital } \\
\text { Market } \\
\text { Accessibility }\end{array}$ & $\begin{array}{c}\text { Openness } \\
\text { Index }= \\
1+(5- \\
\text { KMACC })\end{array}$ & $\begin{array}{c}\text { Equals } 1 \text { if } \\
\text { Member's } \\
\text { Calculated } \\
\text { Quota is Based } \\
\text { on Variants of } \\
\text { the BW Formula }\end{array}$ & $\begin{array}{l}\text { Equals } 1 \text { if } \\
\text { Member } \\
\text { Joined in } \\
\text { the Past } \\
\text { Twenty } \\
\text { Years }\end{array}$ & $\begin{array}{l}\text { Equals } 1 \text { if } \\
\text { Member is a } \\
\text { Developing } \\
\text { Country or a } \\
\text { Transitional } \\
\text { Economy }\end{array}$ \\
\hline Regression Variable Name & $(\mathrm{KMACC})^{\mathrm{l} /}$ & OPEN & NOTBW & MEM20 & DDEV \\
\hline Malawi & 4 & 2 & 1 & 0 & 1 \\
\hline Macedonia, FYR & 3 & 3 & 1 & 1 & 1 \\
\hline Barbados & 3 & 3 & 1 & 0 & 1 \\
\hline Niger & 4 & 2 & 1 & 0 & 1 \\
\hline Estonia & 3 & 3 & 1 & 1 & 1 \\
\hline Mauritania & 4 & 2 & 0 & 0 & 1 \\
\hline Botswana & 3 & 3 & 1 & 0 & 1 \\
\hline Benin & 4 & 2 & 1 & 0 & 1 \\
\hline Burkina Faso & 4 & 2 & 1 & 0 & 1 \\
\hline Chad & 4 & 2 & 1 & 0 & 1 \\
\hline Central African Republic & 4 & 2 & 1 & 0 & 1 \\
\hline Lao, People's Dem. Republic & 4 & 2 & 1 & 0 & 1 \\
\hline Mongolia & 3 & 3 & 1 & 1 & 1 \\
\hline Swaziland & 3 & 3 & 1 & 0 & 1 \\
\hline Albania & 3 & 3 & 1 & 1 & 1 \\
\hline Lesotho & 4 & 2 & 0 & 0 & 1 \\
\hline Equatorial Guinea & 4 & 2 & 0 & 0 & 1 \\
\hline Gambia, The & 4 & 2 & 0 & 0 & 1 \\
\hline Belize & 4 & 2 & 1 & 1 & 1 \\
\hline San Marino & 2 & 4 & 0 & 1 & 0 \\
\hline Vanuatu & 4 & 2 & 1 & 1 & 1 \\
\hline Djibouti & 4 & 2 & 1 & 0 & 1 \\
\hline Eritrea & 3 & 3 & 1 & 1 & 1 \\
\hline St. Lucia & 3 & 3 & 0 & 1 & 1 \\
\hline Guinea-Bissau & 4 & 2 & 1 & 0 & 1 \\
\hline Antigua and Barbuda & 3 & 3 & 0 & 1 & 1 \\
\hline Grenada & 3 & 3 & 1 & 0 & 1 \\
\hline Samoa & 4 & 2 & 0 & 0 & 1 \\
\hline Solomon Islands & 4 & 2 & 1 & 0 & 1 \\
\hline Cape Verde & 4 & 2 & 0 & 0 & 1 \\
\hline Comoros & 4 & 2 & 1 & 0 & 1 \\
\hline St. Kitts and Nevis & 3 & .3 & 0 & 1 & 1 \\
\hline Seychelles & 3 & 3 & 0 & 0 & 1 \\
\hline St. Vincent and the Grenadines & 3 & 3 & 1 & 1 & 1 \\
\hline Dominica & 4 & 2 & 0 & 0 & 1 \\
\hline
\end{tabular}


Table I.8. Qualitative Variables

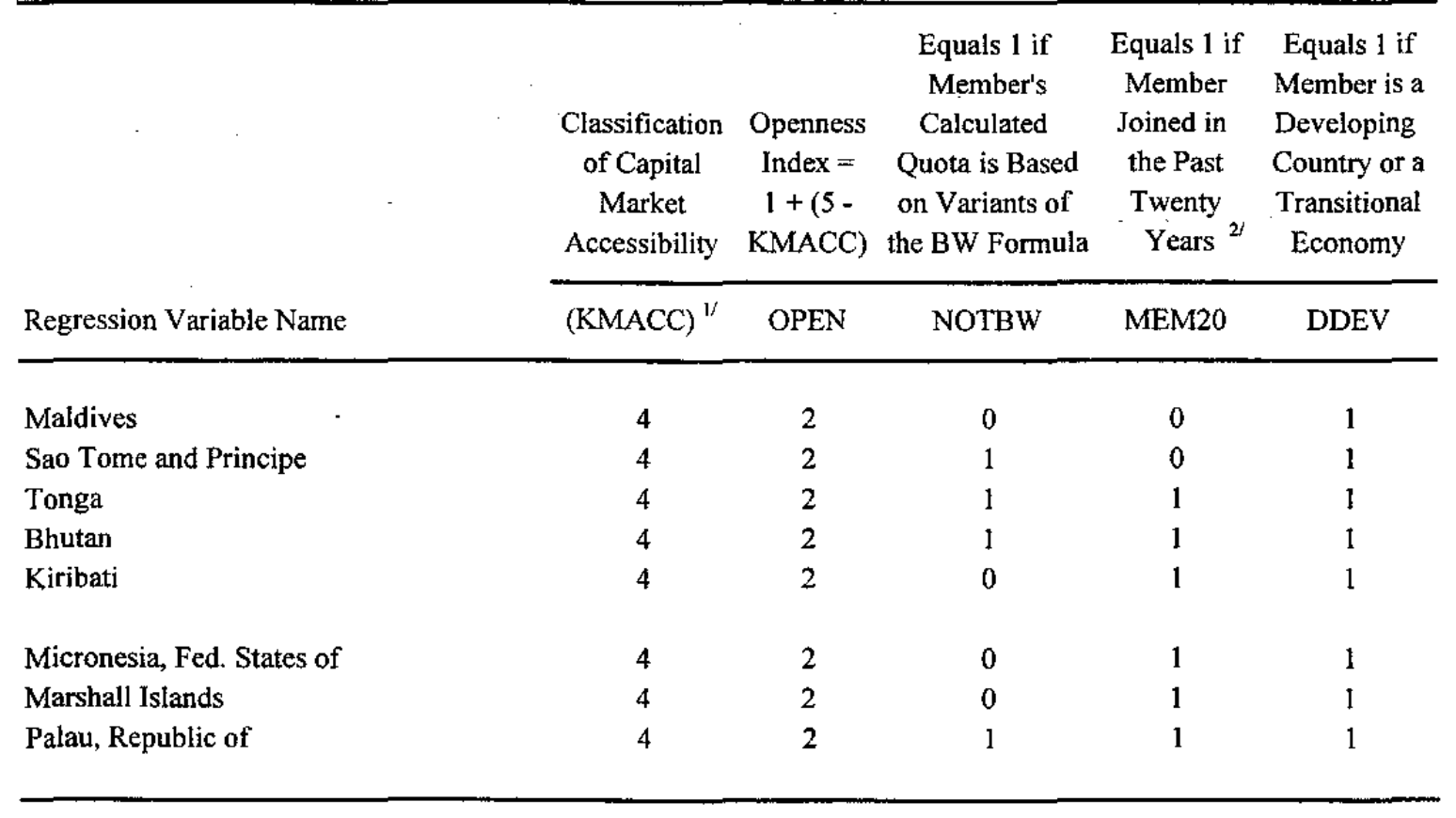

1/ Source: World Economic Outlook; based on WEO classifications where countries with the most access to capital markets are classified as " 1 " while those with the least access are classified as "4". This suggested classification corresponds approximately with those used in EB/CQuota/94/2, except to reflect recent (since the early 1990s) changes in some countries' abilities to access the market; and a reclassification of Canada and Italy to class " 1 ". The variable was used to compute the openness index shown in the next column.

2/ Source: IMF Secretary's Department, Records Division, Membership of the International Monetary Fund (Date of entry into force: December 27, 1945), December 16, 1997. 
Table I. 9. Correlation of Variables (all members)

\begin{tabular}{|c|c|c|c|c|c|c|c|c|}
\hline & GDP & $\begin{array}{c}\text { Variability of } \\
\text { Current } \\
\text { Receipts }\end{array}$ & Reserves & $\begin{array}{c}\text { Current } \\
\text { Payments }\end{array}$ & $\begin{array}{l}\text { Current } \\
\text { Receipts }\end{array}$ & $\begin{array}{c}\text { Normal Capital } \\
\text { Flows }\end{array}$ & $\begin{array}{c}\text { Variability of } \\
\text { Current and } \\
\text { Capital } \\
\text { Account } \\
\text { Receipts }\end{array}$ & Population \\
\hline GDP & 1.0000 & & & & & & & \\
\hline $\begin{array}{l}\text { Variability of Current } \\
\text { Receipts }\end{array}$ & 0.8944 & 1.0000 & & & & & & \\
\hline Reserves & 0.7814 & 0.7697 & 1.0000 & & & & & \\
\hline Current Payments & 0.8994 & 0.9583 & 0.8290 & 1.0000 & & & & \\
\hline Current Receipts & 0.9041 & 0.9454 & 0.8623 & 0.9940 & 1.0000 & & & \\
\hline Normal Capital Flows & 0.4226 & 0.6097 & 0.2201 & 0.5775 & 0.4935 & 1.0000 & & ' \\
\hline Receipts & 0.7766 & 0.8390 & 0.7588 & 0.8884 & 0.8982 & 0.4236 & 1.0000 & \\
\hline Population & 0.2400 & 0.2282 & 0.3168 & 0.2201 & 0.2176 & 0.1343 & 0.1819 & 1.0000 \\
\hline
\end{tabular}




\section{SECTION II. Estimation RESUlts OF Statistical QUOTA Formulas}

This section presents the results of regressions requested by QFRG and analyzed in Chapter V of the report: Detailed results of the regression equations and the resulting quota distributions for members are presented in Statistical Appendix, Part B, Section I. 


\section{A. List of Regression Equations}

1. Re-estimated Bretton Woods Formula for the Whole Membership.

2. Re-estimated Bretton Woods Formula Using PPP-based GDP Instead of GDP at Market Exchange Rates.

3. Linear Bretton Woods Formula With PPP-based GDP Replacing GDP at Market Exchange Rates.

4. Re-estimated Bretton Woods Formula With a Multiplicative Term, Which Includes a Dummy Variable Distinguishing Between Industrial and Developing Countries.

5. Re-estimated Bretton Woods (BW) Formula for Members with Caiculated Quotas Based on the Variants of the BW Formula.

6. Re-estimated Bretton Woods Formula for Members Representing Developing Countries.

7. Re-estimated Bretton Woods Formula for Members with Actual Quota Shares Equal to or Less Than 1 Percent.

8. Re-estimated Bretton Woods Formula for Members Who Joined in the Past Twenty Years.

9. Linear Bretton Woods Formula Without the Multiplicative Factor.

10. Linear Bretton Woods Formula With Current Receipts.

11. Linear Bretton Woods Formula with an Openness Index.

12. Nonlinear Bretton Woods Formula with an Openness Index.

13. Nonlinear Bretton Woods Formula Without the Reserves Variable.

14. Nonlinear Bretton Woods Formula With Gold Reserves Valued at Market Prices.

15. Nonlinear Bretton Woods Formula With a Five Year Average of GDP Replacing the Existing One-year GDP.

16. Nonlinear Bretton Woods Formula With Population.

17. Nonlinear Bretton Woods Formula with Short Term Debt.

18. Nonlinear Bretton Woods Formula With the Variability of External Receipts Replacing the Variability of Current Receipts. 


\section{A. List of Regression Equations}

19. Nonlinear Bretton Woods Formula With the Then-Existing Quota As a Multiplicative Explanatory Variable.

20. Nonlinear Bretton Woods Formula with the Then-Existing Quota As an Additive.Explanatory Variable.

21. Regression of Actual Quotas on Variables Indicative of Ability to Contribute Financial Resources to the Fund.

22. Nonlinear Bretton Woods Formula with a Five-Year Average of GDP, where the Conversion Factors are Centered Five-Year Moving Averages of the Annual Exchange Rates, Replacing the Existing One-Year GDP.

23. Nonlinear Bretton Woods Formula with GNP Converted with the World Bank Atlas Method.

24. Linear Formula with the Then-Existing Quota, Short-Term Debt, Population, and Trade added, and Reserves and Current Payments dropped.

25. Nonlinear Bretton Woods Formula with the Then-Existing Quota As an Additive Explanatory Variable for Countries with Calculated Quotas Based on the Variants of the Bretton Woods Formula.

26. Nested Model Where a Regression of Vulnerability Variables (Represented by the Variability of Current Receipts and Population) is Estimated First.

27. Nested Model Where a Regression of Strength Variables is Estimated First.

28. Linear Estimation of Both Strength and Vulnerability Variables.

29. Re-estimated Bretton Woods Formula With Normal Net Capital Flows as an Additional Variable.

30. Re-estimated Bretton Woods Formula With Real Effective Exchange Rate Variability Times Current Receipts as an Additional Variable.

31. Re-estimated Bretton Woods Formula With Debt as an Additional Variable,

32. Members with Quota Shares of Equal to or Less Than 1.0 Percent. Re-estimated Bretton Woods Formula With Normal Net Capital Flows as an Additional Variable.

33. Members with Quota Shares of Equal to or Less Than 1.0 Percent.

Re-estimated Bretton Woods Formula With Real Effective Exchange Rate Variability Times Current Receipts as an Additional Variable. 


\section{A. List of Regression Equations}

34. Members with Quota Shares of Equal to or Less Than 1.0 Percent. Re-estimated Bretton Woods Formula With Financial Market Accessibility Times Current Payments as an Additional Variable.

35. Members with Quota Shares of Equal to or Less Than 1.0 Percent. Re-estimated Bretton Woods Formula With Debt as an Additional Variable.

36. Re-estimated Bretton Woods Formula With Financial Market Accessibility Times Current Payments as an Additional Variable.

37. Bretton Woods Formula for Schedule A Members Using 1934-43 Data. 


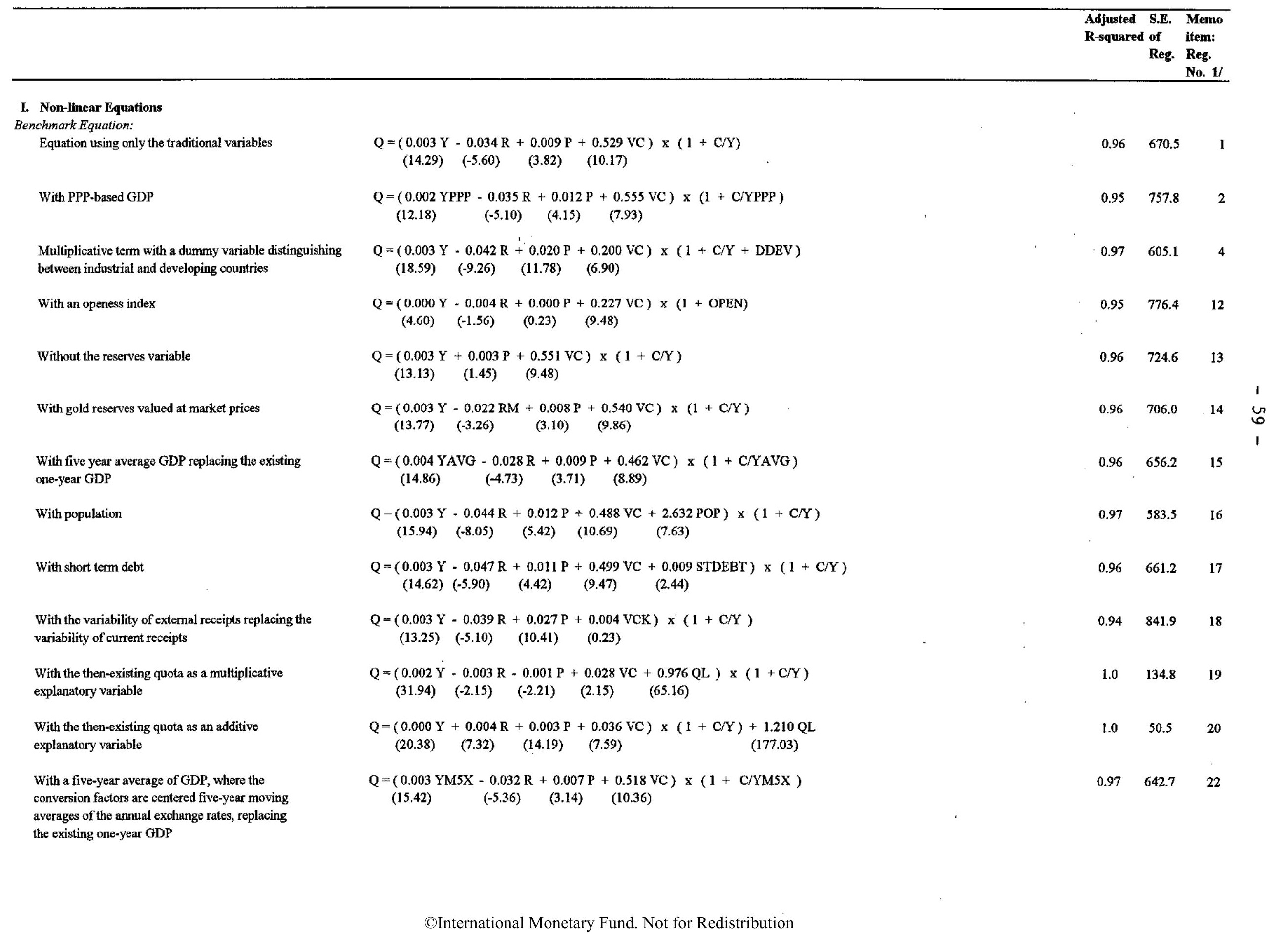




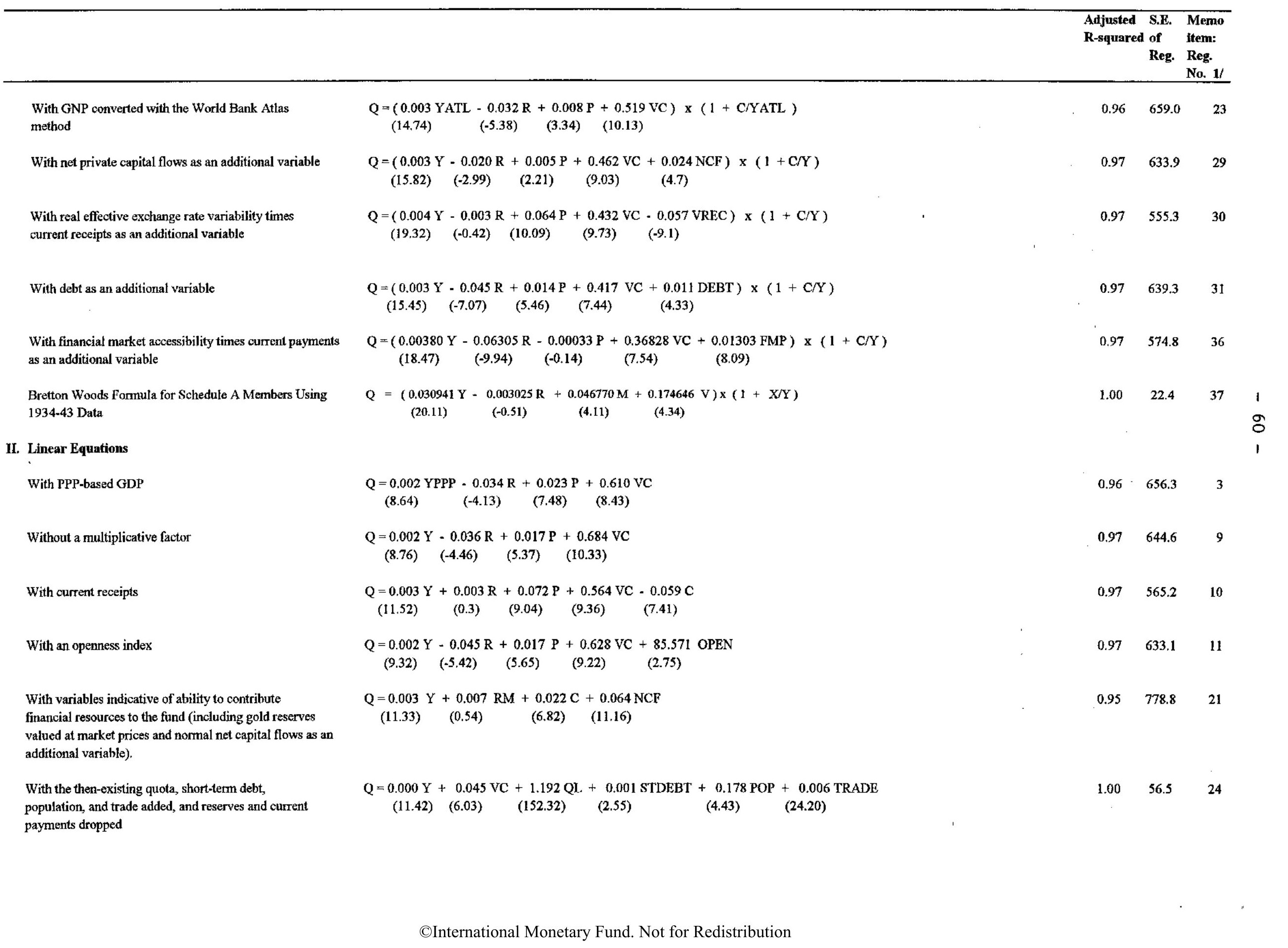




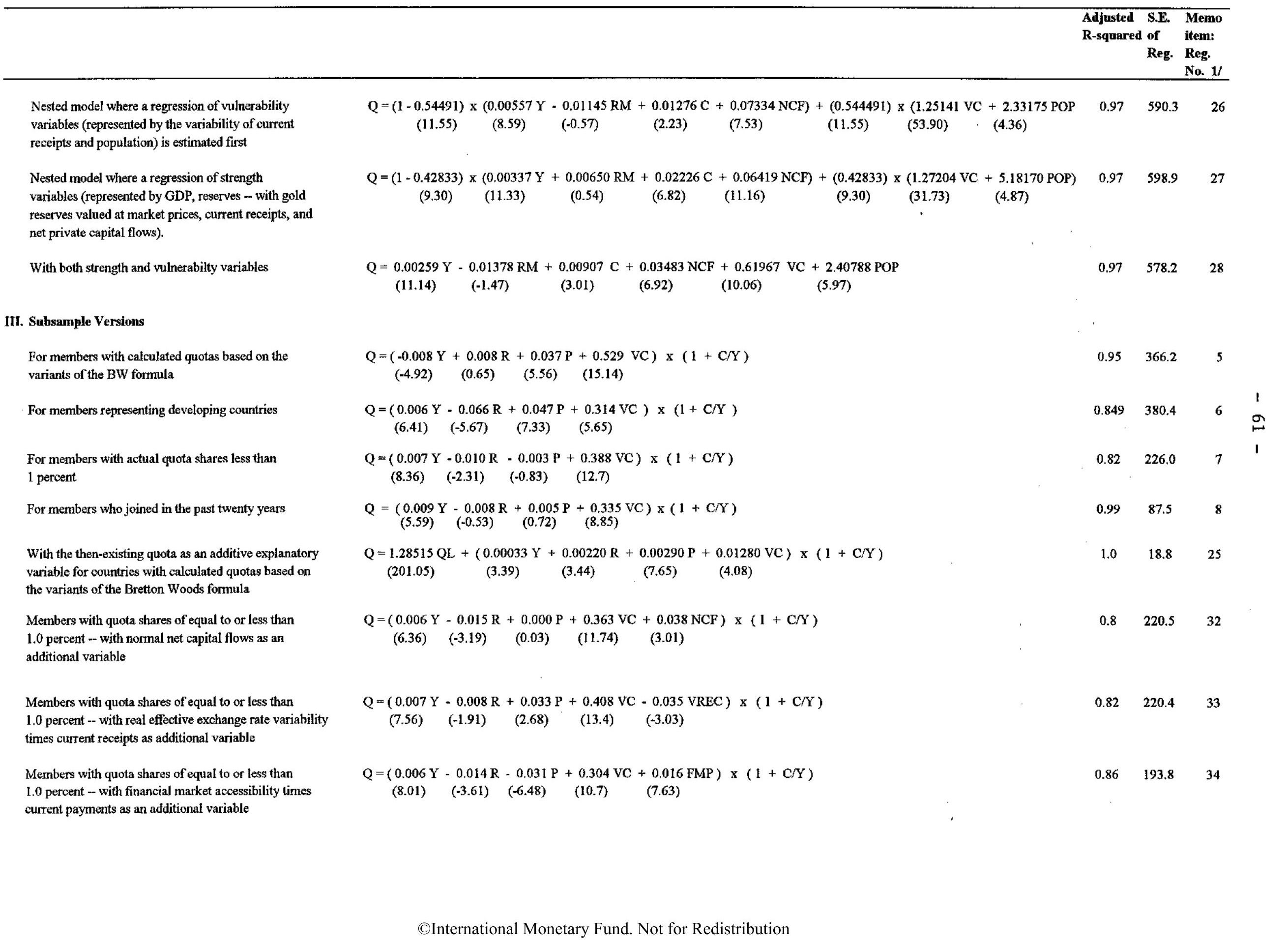




\begin{tabular}{|c|c|c|c|}
\hline & & $\begin{array}{r}\text { Adjusted S.E. } \\
\text { R-squared of } \\
\text { Reg. }\end{array}$ & $\begin{array}{l}\text { Memo } \\
\text { item: } \\
\text { Reg. } \\
\text { No. 1/ } \\
\end{array}$ \\
\hline $\begin{array}{l}\text { Members with quota shares of equal to or less than } \\
1.0 \text { percent -- with debt as an additional variable }\end{array}$ & 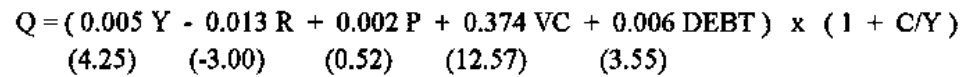 & 218.2 & 35 \\
\hline
\end{tabular}

Note: The variables in the equations are defined as follows:

$Q=$ estimated quota, in millions of SDRs

$\mathrm{Y}=\mathrm{GDP}$ at market exchange rates in 1994

$\mathrm{R}=$ average monthly reserves in 1994

$P=$ annual average current payments over the 1991-1994 (five years) period

$\mathrm{C}=$ annual average current receipts over the 1991-1994 (five years) period

$\mathrm{VC}=$ variability of current receipts, defined as one standard deviation from a five-year moving average over the 1982-1994 (13 years) period

VREC $=$ real effective exchange rate variability times current receipts

POP = population in 1994

DDEV = dummy variable distinguishing between industrial and developing countries. It is equal to $l$ if a country is a developing or a transitional economy

$\mathrm{NCF}=$ normal capital flow proxied by a four-year moving average of actual net private capital flows (inclusive of errors and omissions)

DEBT = total external debt owed to non-residents repayable in foreign currency, goods and services. It is the sum of the public, publicly guaranteed and private non-guaranted

debt, use of IMF credit, and short-term debt

YMSX = is the tive-year average of GDP from 1990 to 1994 where the conversion factor is a centered five-year moving average of the annual exchange rate

STDEBT $=$ short term debt at the end of 1994

OPEN = openness index defined as $1+(5-$ KMACC $)$ KMACC $=$ capital market accessibility, which is based on the WEO classification

$\mathrm{RM}=$ average monthly reserves with gold valued at market rates in 1994

NNKFL $=$ four-year moving average of net private capital flows

$\mathrm{YPPP}=\mathrm{PPP}$-based GDP in 1994

YAVG = five year averages of GDP from 1990-94

VCK = variability of external receipts (the sum of current receipts and capital and financial account credits), defined as one standard deviation from a five-year moving average over the $1982-1994$ (13 years) period

YATL $=1994$ GNP converted using the World Bank Atlas Method

$\mathrm{QL}=$ the then-existing quota

FMP = finacial market accessibility times current payments; financial market accessibility is proxied by a variable which takes values of 4 for developing countries with limited access

to private financial markets, 3 for the rest of developing countries, 2 for industrial countries with easy access to borrowing, and 1 for France, Germany, Japan, the United Kingdom, and the United States

TRADE $=$ average of current payments and receipts over a recent five year pcriod (1991-94)

1/ As numbered in the List of Regression Equations of this section. 
Table II.2. Relative Contributions of Variables to Calculated Quotas 1 / (In percent)

\begin{tabular}{|c|c|c|c|c|c|c|c|c|c|c|c|}
\hline & GDP & Reserves & $\begin{array}{l}\text { Current } \\
\text { Payments }\end{array}$ & $\begin{array}{l}\text { Current } \\
\text { Receipts }\end{array}$ & $\begin{array}{c}\text { Var. } \\
\text { of Curr. } \\
\text { Receipt }\end{array}$ & $\begin{array}{l}\text { Ratio of } \\
\text { Current } \\
\text { Recetpts } \\
\text { to GDP } \\
\end{array}$ & $\begin{array}{c}\text { New } \\
\text { variable }\end{array}$ & $\begin{array}{c}\text { New } \\
\text { variable }\end{array}$ & $\begin{array}{c}\text { New } \\
\text { variable }\end{array}$ & $\begin{array}{c}\text { New } \\
\text { variable }\end{array}$ & $\begin{array}{l}\text { Memo } \\
\text { item: } \\
\text { Regres } \\
\text { no. 2l } \\
\end{array}$ \\
\hline I. Non-linear Equations & & & & & & & & & & & \\
\hline Benchmark Equation: & & & & & & & & ' & & & \\
\hline Equation using only the traditional variables & 27.5 & -13.7 & 17.8 & - & 47.6 & 20.8 & - & -- & - & , $\cdots$ & 1 \\
\hline Memo: Existing five formulas & 28.9 & 3.9 & 33.3 & 4.4 & 13.4 & 16.1 & & & & & \\
\hline With PPP-based GDP & 28.1 & -13.0 & 21.2 & -- & 45.7 & 18.0 & -. & -. & - & - & 2 \\
\hline $\begin{array}{l}\text { Multiplicative term with a dummy variable distinguishing } \\
\text { between industrial and developing countries }\end{array}$ & 29.3 & -16.0 & 37.5 & - & 17.2 & $323 /$ & - & - & - & - & 4 \\
\hline With an openess index & 3.1 & -1.6 & 0.5 & -- & 22.6 & - & 75.5 & -- & -- & -- & 12 \\
\hline Without the reserves variable & 25.8 & -- & 6.2 & -- & 46.8 & 21.2 & -- & -- & - & -- & 13 \\
\hline With gold reserves valued at market prices & 27,4 & -11.1 & 15.4 & - & 47.3 & 21.0 & - & -- & -- & -- & 14 \\
\hline $\begin{array}{l}\text { With five year average GDP replacing the existing } \\
\text { one-year GDP }\end{array}$ & 32.4 & -11.3 & 16.8 & -- & 41.3 & 20.8 & - & - & - & - & 15 \\
\hline With population & 25.8 & -17.0 & 21.4 & - & 42.2 & 20.4 & 7.2 & -- & -- & $-\cdot$ & 16 \\
\hline With short-term debt & 29.0 & -18.9 & 21.4 & 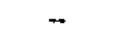 & 45.2 & 20.9 & 2.3 & - & - & -- & 17 \\
\hline $\begin{array}{l}\text { With the variability of external receipts replacing the } \\
\text { variability of current receipts }\end{array}$ & 36.6 & -18.0 & 60.6 & - & 0.8 & 19.9 & -- & -- & - & $\cdot$ & 18 \\
\hline $\begin{array}{l}\text { With the then-existing quota as a multiplicative } \\
\text { explanatory variable }\end{array}$ & 12.7 & -1.0 & -2.0 & - & 2.3 & 20.9 & 67.1 & - & -- & 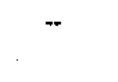 & 19 \\
\hline $\begin{array}{l}\text { With the then-existing quota as an additive } \\
\text { explanatory variable }\end{array}$ & 3.7 & 1.4 & 4.6 & -- & 3.0 & 3.4 & 83.9 & - & -- & -- & 20 \\
\hline
\end{tabular}


Table II.2. Relative Contributions of Variables to Calculated Quotas $1 /$

(In percent)

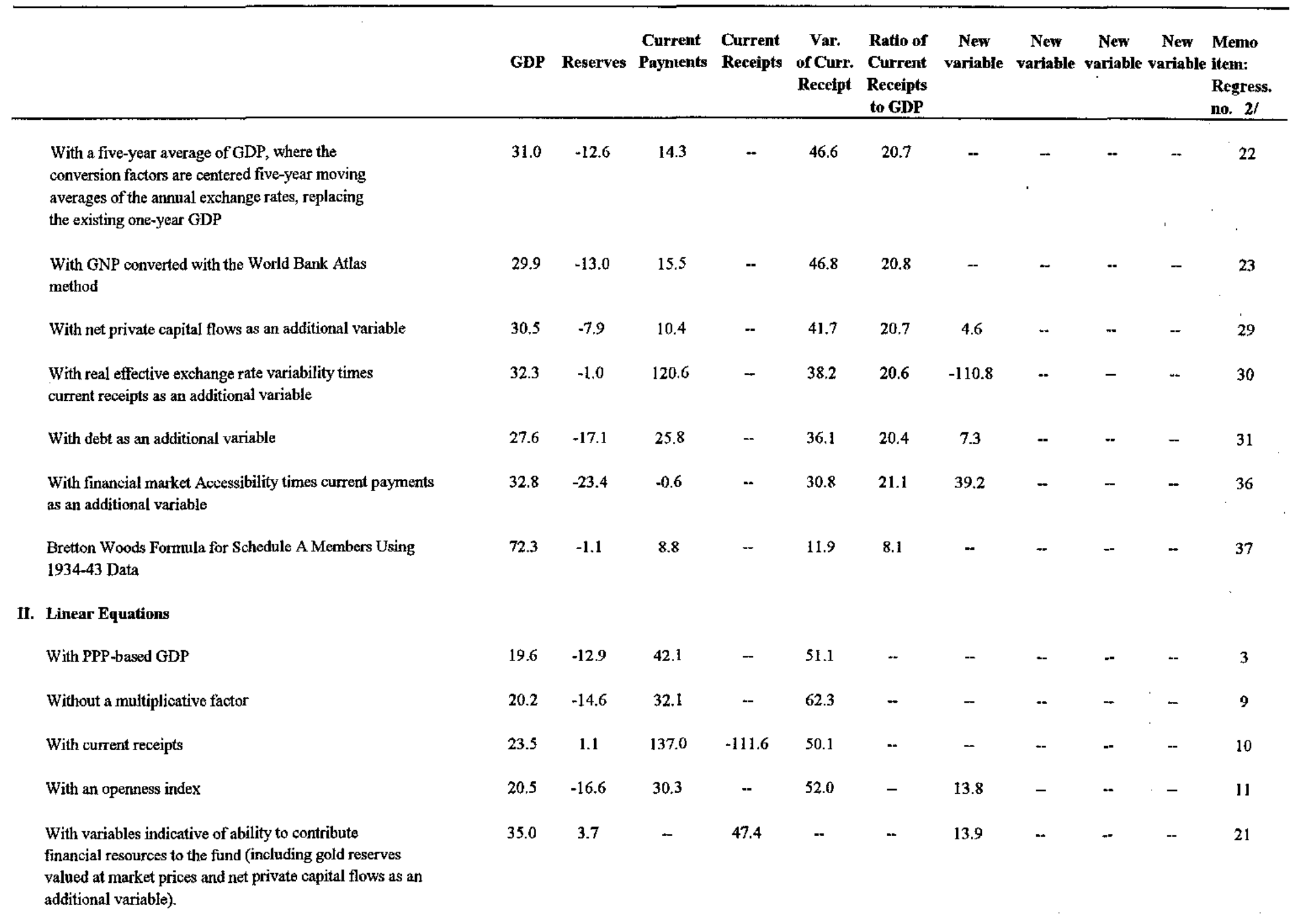


Table II.2. Relative Contributions of Variables to Calculated Quotas $1 /$

(In percent)

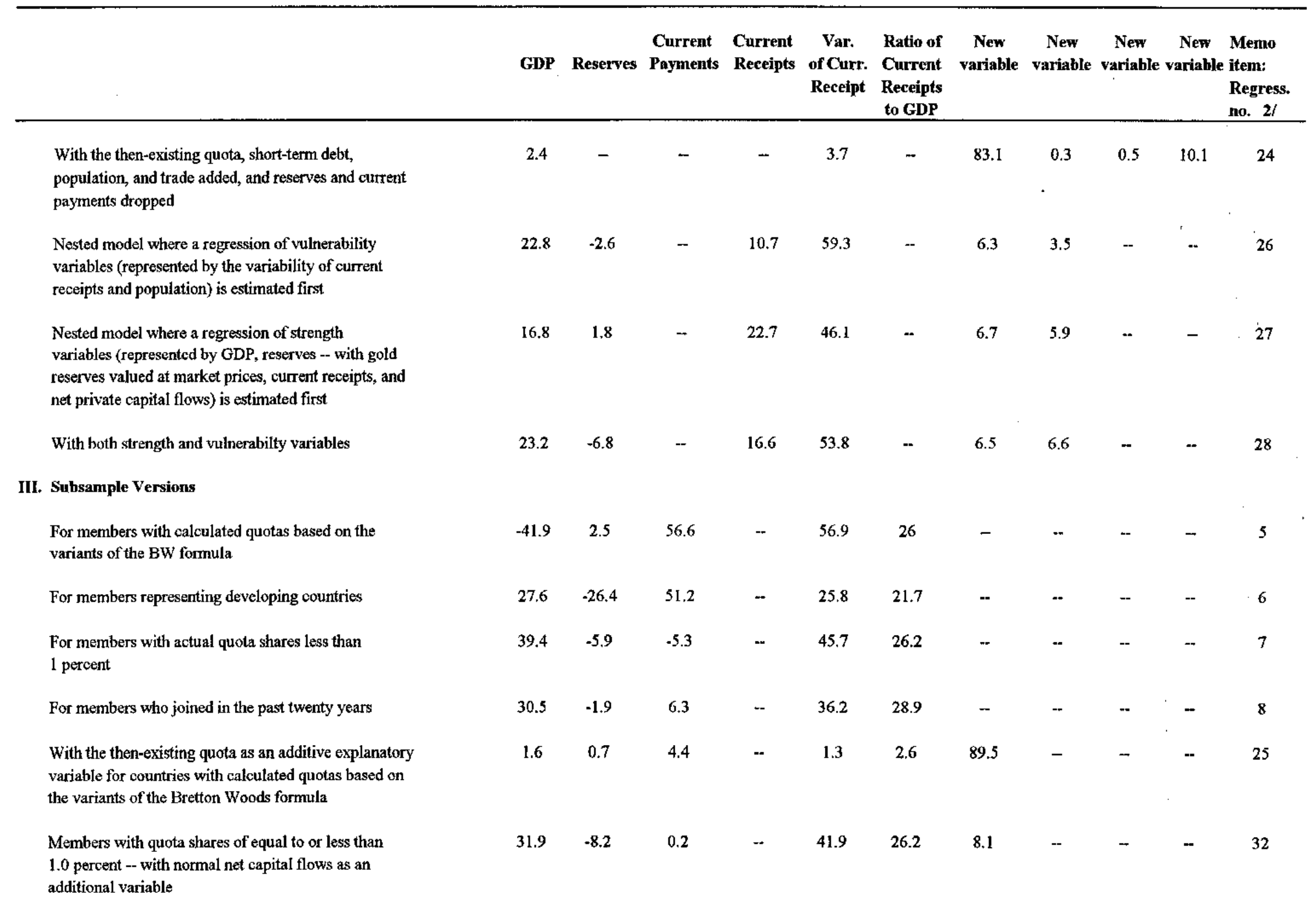


Table II.2. Relative Contrihutions of Variables to Calculated Quotas $1 /$

(in percent)

\begin{tabular}{|c|c|c|c|c|c|c|c|c|c|c|c|}
\hline & GDP & Reserves & $\begin{array}{l}\text { Current } \\
\text { Payments }\end{array}$ & $\begin{array}{l}\text { Current } \\
\text { Receipts }\end{array}$ & $\begin{array}{l}\text { Var. } \\
\text { of Curr. } \\
\text { Receipt }\end{array}$ & $\begin{array}{l}\text { Ratio of } \\
\text { Current } \\
\text { Receipts } \\
\text { to GDP } \\
\end{array}$ & $\begin{array}{c}\text { New } \\
\text { variable }\end{array}$ & $\begin{array}{c}\text { New } \\
\text { variable }\end{array}$ & $\begin{array}{c}\text { New } \\
\text { variahle }\end{array}$ & $\begin{array}{c}\text { New } \\
\text { variable }\end{array}$ & $\begin{array}{l}\text { Memo } \\
\text { item: } \\
\text { Regress. } \\
\text { no. } 2 /\end{array}$ \\
\hline $\begin{array}{l}\text { Members with quota shares of equal to or less than } \\
1.0 \text { percent -- with real effective exchange rate variability } \\
\text { times current receipts as additional variable }\end{array}$ & 34.7 & -4.7 & 58.1 & - & 46.6 & 26.1 & -60.7 & -- & -. & -- & 33 \\
\hline $\begin{array}{l}\text { Members with quota shares of equal to or less than } \\
1.0 \text { percent - with financial market accessibility times } \\
\text { current payments as an additional variable }\end{array}$ & 30.5 & -7.4 & -51.4 & - & 33.0 & 26.3 & 69.0 & -- & - & -. & 34 \\
\hline $\begin{array}{l}\text { Members with quota shares of equal to or less than } \\
1.0 \text { percent - with debt as an additional variable }\end{array}$ & 24.6 & -7.3 & 3.3 & - & 42.5 & 26.2 & 10.7 & -. & -. & - & 35 \\
\hline
\end{tabular}

Note: The variables in the equations are defined in Table Il.1.

1/ The relative contribution of a variable to the calculated quota is the ratio between the variable times its coefficient and the members' calculated quota. The contribution of the ratio of current receipts to GDP is the contribution of the nonlinear element to the calculated quotas, i.e., the extent to which the calculated quota is revised by the application of the multiplier (unity plus the ratio of current receipts to GDP).

2/ As numbered in the List of Regression Equations in the beginning of this section.

3/ It includes the contribution of the dummy variable. 
Table II.3. Estimated Quota Shares by WEO Classification 1/

(In percent)

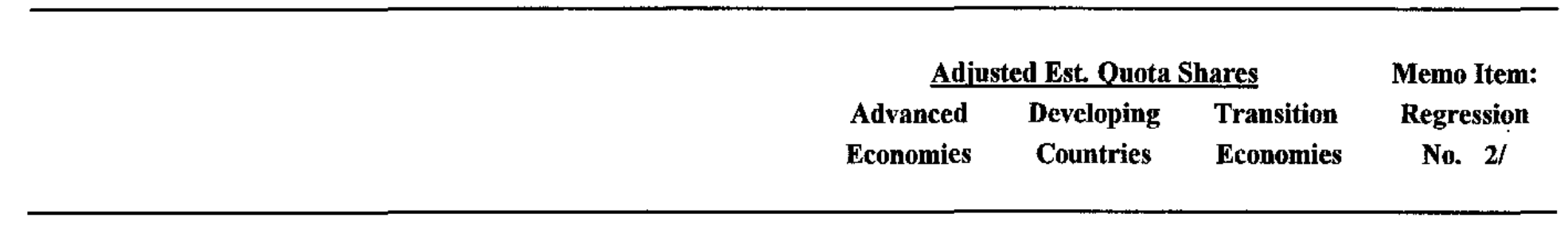

\section{Non-linear Equations}

Benchmark Equation:

Equation using only the traditional variables

Memo: Existing five formulas,

Existing quotas

With PPP-based GDP

Multiplicative term with a dummy variable distinguishing

between industrial and developing countries

With an openess index

Without the reserves variable

With gold reserves valued at market prices

With five year average GDP replacing the existing

one-year GDP

With population

With short-term debt
69.54

21.43

9.03

5.3

75.48

19.22

29.21

7.6

63.20

65.01

26.27

7.74

65.64

25.28

9.08

4

73.80

18.28

7.93

12

67.73

23.54

8.74

68.67

22.35

8.98

69.31

21.30

9.40

65.80

25.22

8.98

16

70.11

21.05

8.85
1 
Table II.3. Estimated Quota Shares by WEO Classification 1/

(In percent)

\begin{tabular}{|c|c|c|c|c|}
\hline & \multicolumn{3}{|c|}{ Adiusted Est. Quota Shares } & \multirow{2}{*}{$\begin{array}{c}\text { Memo Item: } \\
\text { Regression } \\
\text { No. 2/ }\end{array}$} \\
\hline & $\begin{array}{l}\text { Advanced } \\
\text { Economies }\end{array}$ & $\begin{array}{l}\text { Developing } \\
\text { Countries }\end{array}$ & $\begin{array}{l}\text { Transition } \\
\text { Economies }\end{array}$ & \\
\hline $\begin{array}{l}\text { With the variability of external receipts replacing the } \\
\text { variability of current receipts }\end{array}$ & 81.91 & 13.93 & 4.16 & 18 \\
\hline $\begin{array}{l}\text { With the then-existing quota as a multiplicative } \\
\text { explanatory variable }\end{array}$ & 63.28 & 28.62 & 8.10 & 19 \\
\hline $\begin{array}{l}\text { With the then-existing quota as an additive } \\
\text { explanatory variable }\end{array}$ & 63.58 & 28.81 & 7.61 & 20 \\
\hline $\begin{array}{l}\text { With a five-year average of GDP, where the } \\
\text { conversion factors are centered five-year moving } \\
\text { averages of the anmual exchange rates, replacing } \\
\text { the existing one-year GDP }\end{array}$ & 69.43 & 21.71 & 8.86 & 22 \\
\hline $\begin{array}{l}\text { With GNP converted with the World Bank Atlas } \\
\text { method }\end{array}$ & 69.55 & 21.32 & 9.13 & 23 \\
\hline With normal net capital flows as an additional variable & 69.43 & 22.38 & 8.19 & 29 \\
\hline $\begin{array}{l}\text { With real effective exchange rate variability times } \\
\text { current receipts as an additional variable }\end{array}$ & 68.70 & 23.36 & 7.94 & 30 \\
\hline With debt as an additional variable & 66.24 & 24.99 & 8.78 & 31 \\
\hline
\end{tabular}


Table II.3, Estimated Quota Shares by WEO Classification 1/

(In percent)

\begin{tabular}{|c|c|c|c|c|c|}
\hline & & \multicolumn{3}{|c|}{ Adiusted Est. Quota Shares } & \multirow{2}{*}{$\begin{array}{l}\text { Memo Item: } \\
\text { Regression } \\
\text { No. } 2 i\end{array}$} \\
\hline & & $\begin{array}{l}\text { Advanced } \\
\text { Economies }\end{array}$ & $\begin{array}{l}\text { Developing } \\
\text { Countries }\end{array}$ & $\begin{array}{l}\text { Transition } \\
\text { Economies }\end{array}$ & \\
\hline & $\begin{array}{l}\text { With financial market accessibility times current payments } \\
\text { as an additional variable }\end{array}$ & 66.16 & 24.67 & 9.16 & 36 \\
\hline & $\begin{array}{l}\text { Bretton Woods Formula for Schedule A Members Using } \\
\text { 1934-43 Data }\end{array}$ & & & & \\
\hline H. & Linear Equations & & & & \\
\hline & With PPP-based GDP & 65.95 & 25.20 & 7.86 & 3 \\
\hline & Without a multiplicative factor & 69.78 & 21.65 & 8.57 & 9 \\
\hline & With current receipts & 68.55 & 23.99 & 7.46 & 10 \\
\hline & With an openness index & 64.59 & 25.86 & 9.55 & 11 \\
\hline & $\begin{array}{l}\text { With variables indicative of ability to contribute } \\
\text { financial resources to the fund (including gold reserves } \\
\text { valued at market prices and normal net capital flows as an } \\
\text { additional variable). }\end{array}$ & 77.87 & 18.73 & 3.40 & 21 \\
\hline & $\begin{array}{l}\text { With the then-existing quota, short-term debt, } \\
\text { population, and trade added, and reserves and current } \\
\text { payments dropped }\end{array}$ & 63.67 & 28.76 & 7.57 & 24 \\
\hline
\end{tabular}




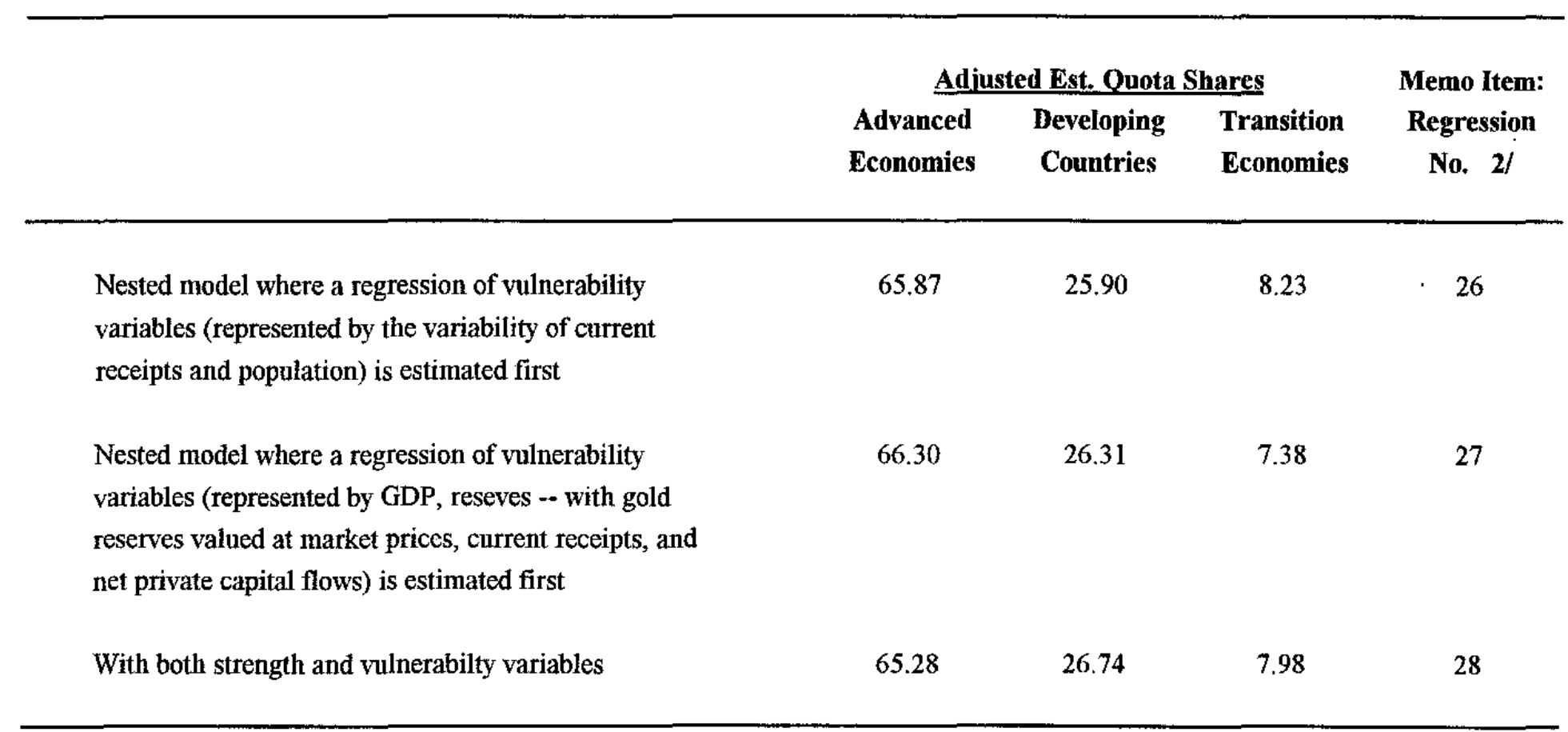

1/ According to the WEO country classifications, advanced economies include the industrial countries of North America and Europe, Japan, and two newly industrialized Asian economies (Korea and Singapore). The countries in transition include the 15 members that were formerly part of the Soviet Union, the successor countries to the former Yugoslavia and Czechoslovakia, Albania, Hungary, Poland, Romania, and Mongolia. The rest of the members are classified as developing countries.

2/ As numbered in the List of Regression Equations in the beginning of this section. 


\section{Section III. Chow and Wald Tests for the Stability of Coefficient Estimates}

This section presents the results of Wald and Chow tests for the stability of the estimated coefficients of the Bretton Woods formula, using data from the Sixth to the Eleventh Reviews, including explanatory notes.

Part A presents the Chow test results on the stability of the coefficient estimates of the Bretton Woods formula, using data from the Sixth to the Eleventh Reviews, including the methodology used in performing the Chow tests.

The results of these statistical tests suggest instability in the coefficients of the Bretton Woods formula. The best result seems to come from the pair-wise test of the Seventh and Eighth Reviews, which could perhaps be attributed to two factors: (1) the short time period in between these reviews because the Eighth Review was accelerated; and (2) the relatively large selective element of the Eighth Review, which allows the underlying economic variables to have a somewhat greater influence on the outcome of the Eighth Review.

Part B presents the results of tests for the stability of the coefficient estimates of the Bretton Woods formula, using data from the Sixth to the Eleventh Reviews, while taking into account the systematic tendency of actual quotas to fall over time in relation to GDP or external trade.

The significance of the tendency of actual quotas to fall in relation to GDP or external trade over the Sixth to the Eleventh Reviews is examined by pair-wise testing ( $F$-tests) of whether the variances of the error terms from the estimated Bretton Woods formula over these reviews are equal. If the error variance is the same over a pair of reviews, then the Chow test remains an appropriate test. If not, we need to use other statistics, like the Wald test.

The formal tests for the equality of variances between reviews show that the error variances for the rolling (or pair-wise comparisons of) Sixth and Seventh, Seventh and Eighth, Ninth and Tenth, and Tenth and Eleventh Reviews are statistically different. This implies that the Chow test for these pairs is inappropriate. Nonetheless, the alternative Wald test indicates that the coefficients generated by the Bretton Woods formula are not stable for these pairwise comparisons of quota review periods. For the pair of the Eighth and Ninth Reviews, however, the results of the Chow test in Part A are valid.

In sum, regardless of whether we can use the Chow test or have to use the Wald test, the statistical tests suggest instability of the coefficient estimates of the Bretton Woods formula. A detailed list of the regressions performed is presented in Statistical Appendix, Part B, Section II. 


\section{A. Chow Tests}

1. To test the stability of coefficients in the reduced Bretton Woods formula over the Sixth to Eleventh Reviews, we used the Chow test for (1) rolling consecutive pairs of reviews, starting with the Sixth Review (Table III.1.1), and (2) cumulative reviews, starting with the Sixth Review (Table III.1.2).

2. To perform the Chow tests, we used a constant-membership sample of 121 members participating in the Sixth Review plus China. To test, for example, the stability of the coefficient estimates under the Sixth and Seventh Reviews, we run the following regressions:

$$
\begin{aligned}
& Q=\left(a^{l} Y+b^{l} R+c^{l} P+d^{l} V C\right) x(1+C / Y) \quad \text { for the Sixth Review } \\
& Q=\left(a^{2} Y+b^{2} R+c^{2} P+d^{2} V C\right) x(1+C / Y) \quad \text { for the Seventh Review }
\end{aligned}
$$

If $a^{l}=a^{2} \quad b^{1}=b^{2} \quad c^{l}=c^{2} \quad d^{l}=d^{2}$, then we can estimate a common relationship for the entire (pooled) data, i.e.

$$
Q=(a Y+b R+c P+d V C) \times(I+C / Y) \quad \text { for the Sixth and Seventh Reviews }
$$

These four linear restrictions on $a, b, c$, and $d$ can be tested using the $\mathrm{F}$ test. The $\mathrm{F}$ test is

where

$$
F=\frac{\left(S S R_{p}-S S R_{s}\right) /(4+1)}{S S R_{s} /(122+122-2 \times 4-2)}
$$

$$
\begin{aligned}
& S S R=\text { Sum of squared residuals } \\
& S S R_{p}=S S R \text { of the pooled data }=S S R_{6 t h R} \text { and } 7 t h R \\
& S S R_{s}=S S R_{6 t h R}+S S R_{7 t h R}
\end{aligned}
$$

which has an $\mathrm{F}$ distribution with degrees of freedom $(4+1),(122+122-2 \times 4-2)$.

3. If the $F$ value is less than the critical value of 2.25 from the $F$ tables at the 5 percent significance level, i.e., the calculated $\mathrm{F}$ value is not significant at the 5 percent level, we do not reject the null hypothesis that the relationship is stable (see Madala, G. S., Econometrics, 1977, McGrow-Hill, pp. 198-199).

4. The results shown in Tables III.1.1 and III.1.2 indicate, at the 5 percent significance level, rejection of the hypothesis that the coefficients of the Bretton Woods formula are stable over time. 
Table III.1.1 : Chow Tests (Rolling)

\begin{tabular}{lr}
\hline & F-Statistic \\
\hline Sixth and Seventh Reviews & $14.22 *$ \\
Seventh and Eighth Reviews & $3.67 *$ \\
Eighth and Ninth Reviews & $20.65 *$ \\
Ninth and Tenth Reviews & $40.27 *$ \\
Tenth and Eleventh Reviews & $12.35 *$ \\
\hline
\end{tabular}

Note: The asterisk indicates significance of the $5 \%$ significance level.

Table III.1.2 : Chow Tests (Cumulative)

\begin{tabular}{lr}
\hline & F-Statistic \\
\hline Sixth and Seventh Reviews & $14.22 *$ \\
Seventh and Eighth Reviews & $22.92 *$ \\
Eighth and Ninth Reviews & $76.60 *$ \\
Ninth and Tenth Reviews & $188.47 *$ \\
Tenth and Eleventh Reviews & $127.58 *$ \\
\hline
\end{tabular}

Note: The asterisk indicates significance of the $5 \%$ significance level. 


\section{B. Wald Tests: Tests of Structural Change with Unequal Variances}

In using the Chow test, an important assumption made is that the error variance is the same in all regressions. If this is not true, the error variance for one quota review period is $\sigma_{1}^{2}$, while that for the next review period is $\sigma_{2}^{2}$, and so on, in the restricted (two-reviews combined) model. The restricted model is, therefore, heteroscedastic, and the results from applying the non-linear Bretton Woods formula to such a model present problems of statistical inference. ${ }^{1}$. In this case, it has been argued that it is likely that we overestimate the significance level of our test statistic. ${ }^{2}$ In other words, the calculated $F$ statistic is biased upward and indicates greater instability in the coefficient estimates than in fact exists.

To deal with this problem, we estimate all separate regressions and examine the estimates of the error variances. To test for significant differences, we use pairwise $F$-tests. ${ }^{3}$ Without any significant difference, we proceed with Chow tests. If, however, there is evidence to suggest that the variances are actually different, we may explicitly estimate the model, accounting for the heteroscedasticity. However, if the sample is reasonably large, we may use the Wald test that is valid whether or not the error variances are the same. To set up this test, we suppose that $\hat{\theta}_{1}$ and $\hat{\theta}_{2}$ are two normally distributed estimators of a parameter based on independent samples, ${ }^{4}$ with variance matrices $V_{1}$ and $V_{2}$. Then, under the null hypothesis that the two estimates have the same expected value, i.e., there is no structural change between the two quota reviews,

$$
\hat{\theta}_{1}-\hat{\theta}_{2} \text { has mean } 0 \text { and variance } V_{1}+V_{2}
$$

\footnotetext{
${ }^{1}$ In particular, heteroscedasticity exists whenever the variance of the error term changes across different segments of the population, which are determined by the different values of the explanatory variables (Wooldridge, Jeffrey, M., 2000, Introductory Econometrics: A Modern Approach, South-Western College Publishing, Thomson Learning, United States, p. 248). In the case of the estimated Bretton Woods formula, heteroscedasticity is present if the variance of the error term increases with the factors affecting actual quotas, i.e., GDP, trade, reserves, and variability.

2 Toyoda, Toshihisa, 1974, "Use of the Chow Test Under Heteroscedasticity," Econometrica, Vol. 42, No. 3, May, pp. 601-8; and Schmidt, Peter and Robin Sickles, 1977, "Some Further Evidence on the Use of the Chow Test Under Heteroscedasticity," Econometrica, Vol. 45, No. 5, July, pp. 1293-98.

${ }^{3}$ The $F$-test is used for variance equality tests with two subgroups $(G=2)$. We compute the variance for each subgroup and denote the subgroup with the larger variance as $L$ and the subgroup with the smaller variance as $S$. Then the $F$-statistic is given by $F=s_{L}^{2} / s_{S}^{2}$ where $s_{g}^{2}$ is the variance in subgroup $g=L, S$. This $F$-statistic has an $F$-distribution with $n_{L}-1$ numerator degrees of freedom and $n_{S}-1$ denominator degrees of freedom under the null hypothesis of equal variance and independent normal samples.

${ }^{4}$ Without independence, this test fails.
} 
and the Wald statistic,

$$
W=\left(\hat{\theta}_{1}-\hat{\theta}_{2}\right)^{\prime}\left(V_{1}+V_{2}\right)^{-1}\left(\hat{\theta}_{1}-\hat{\theta}_{2}\right)
$$

has a chi-squared distribution with $K$ degrees of freedom. A test that the difference between the parameters is zero can be based on this statistic. It is straightforward to apply this to our test of common parameter vectors in our regressions. Large values of the statistic lead us to reject the hypothesis of no difference (or of stability in the coefficients). Note that we base such a test on estimates of $V_{1}$ and $V_{2}$. The test is valid in large samples, so we may use our least squares estimates of the two covariance matrices to compute $W^{5}$

As shown in the attached tables, the $F$-test results indicate that the null hypothesis (that the error variance from the estimated Bretton Woods formula is equal over rolling pairs of reviews, from the Sixth through the Eleventh Reviews) is rejected for the Sixth and Seventh, Seventh and Eighth, Ninth and Tenth, and Tenth and Eleventh Reviews (Table III.2.1). Therefore, Chow tests are not appropriate for testing the stability of coefficients of the Bretton Woods formula over these review periods. Nonetheless, application of the Wald test suggests that the coefficient estimates of the Bretton Woods formula are not stable over these reviews (Wald test values exceed the critical $x^{2}$ value (4 restrictions) of 9.49 , at the 5 percent significance level, for all such pairs of reviews-Table III.2.2). However, the pair-wise comparison of the Eighth and Ninth Reviews suggests that the corresponding error variances are equal, and therefore the Chow test results are valid (Table III.2.3).

${ }^{5}$ See Greene, William, H., 1993, Econometric Analysis, 2d edition, Prentice Hall, Englewood Cliffs, N. J., pp. 215-6. 
Table III.2.1 : Test for Equality of Variances of Error Terms (F-test)

\begin{tabular}{ll}
\hline & F-Statistic \\
\hline Sixth and Seventh Reviews & $3.85 *$ \\
Seventh and Eighth Reviews & $1.58 *$ \\
Eighth and Ninth Reviews & $1.16^{*}$ \\
Ninth and Tenth Reviews & $1.59 *$ \\
Tenth and Eleventh Reviews & $2.77^{*}$ \\
\hline
\end{tabular}

Note: An asterisk indicates significance at the 5\% level. The critical values of the F-statistic (120.120) are 1.53,1.35, and 1.26 at the $1 \%$ level, $5 \%$, and $10 \%$ significance levels, respectively.

Table III.2.2 : Wald Test

\begin{tabular}{lr}
\hline & Chi-square Statistic \\
\hline Sixth and Seventh Reviews & $97.73^{*}$ \\
Seventh and Eighth Reviews & $20.35^{*}$ \\
Ninth and Tenth Reviews & $216.02^{*}$ \\
Tenth and Eleventh Reviews & $59.26^{*}$ \\
\hline
\end{tabular}

Note: An asterisk indicates significance at the $5 \%$ level. The critical values of the Chi-square statistic for 4 restrictions are $13.28,9.49$, and 7.78 at the $1 \%$, $5 \%$, and $10 \%$ significance levels, respectively.

Table III.2.3 : Chow Tests

\begin{tabular}{lc}
\hline & F-Statistic \\
\hline Eighth and Ninth Reviews & $20.65^{*}$ \\
\hline
\end{tabular}

Note: An asterisk indicates significance at the $5 \%$ level. The critical values of the F-statistic $(5.200)$ are $3.11,2.66$, and 1.88 for the $1 \%, 5 \%$, and $10 \%$ significance levels, respectively. 


\section{SEction IV. Nested Formulas With Vulnerability and Strength VARIABLes}

This section presents the methodology and statistical results from applying the nested model to certain vulnerability and strength variables.

Part A presents the Davidson-MacKinnon J test used in estimating the relative weights of strength and vulnerability variables in a two-equation formula system.

Part B presents a summary table and regression results from nested models used to estimate the relative weights of strength and vulnerability variables in the determination of actual quotas.

The regression results for the nested model where the vulnerability model is estimated first (Regression No. 53) indicate that the relative weight for the vulnerability variables $(\alpha)$ is 0.54 , while that for the nested model where the strength model is estimated first (Regression No. 54) indicate that the relative weight for the vulnerability variables $(\alpha)$ is 0.43 . Since the $t$ statistics for the relative weights in both regressions are statistically significant, neither regression can be rejected. The relative contributions of variables in these regressions, based on the weighted coefficients, indicate that the relative contribution of $\mathrm{Y}$ is around 20 percent on average, and that of $\mathrm{VC}$ is over 50 percent on average.

The regression equations and resulting quota distributions for members from the nested formulas is presented in Statistical Appendix, Part B, Section III. 


\section{A. Davidson-MacKinnon J Test}

To estimate the relative weights of strength and vulnerability variables in a two-equation formula system, we used the Davidson-MacKinnon $\mathrm{J}$ test. The $\mathrm{J}$ test proceeds as follows:

1. Assume the following two models explain actual quotas:

Model A with vulnerability variables: $\quad Y=Z \gamma+v$

where $Y=$ actual quotas, $Z=$ vulnerability variables, $\gamma=$ coefficients of $Z$, and $v=$ error term

Model B with strength variables: $\quad Y=X \beta+u$ where $X=$ strength variables, $\beta=$ coefficients of $X$, and $u=$ error term.

Models A and B are nonnested if one cannot be derived as a special case of the other. To test whether the models are nonnested, we estimate the artificially nested model $\mathrm{C}$, and then test one or both of the original models against it:

Model C, nesting or encompassing models $\mathrm{A}$ and $\mathrm{B}$ :

$$
Y=(1-\alpha) X \beta+\alpha Z \gamma+w
$$

where $\alpha=$ relative weight of vulnerability variables, with $0 \leq \alpha \leq 1$, and $w=$ error term.

2. Davidson-MacKinnon J test. Since model $\mathrm{C}$ is not estimable, because the parameters $\alpha, \beta$, and $\gamma$ are not separately identifiable, Davidson and MacKinnon ${ }^{1}$ suggested that model $\mathrm{C}$ be replaced by one in which the unknown parameters of the model that is not being tested are replaced by consistent estimates of those parameters. The idea is that if one model is the correct model, then the fitted values from the other model should not have further explanatory power when estimating that model. Thus, the $J$ testing procedure follows the steps:

(a) Estimate (by OLS) model A and obtain the estimated (fitted) $Y$ values, $\hat{Y}^{A}$.

(b) Add $\hat{Y}^{A}$ of step 1 as an additional regressor to model B and estimate (by OLS) the following model D:

Model D:

$$
Y=X \delta+\alpha \hat{Y}^{A}+w_{I}
$$

\footnotetext{
${ }^{1}$ Davidson, R. and J.G. MacKinnon, 1981, "Several Tests for Model Specifications in the Presence of Alternative Hypotheses," Econometrica, Vol. 49. pp. 781-93.
} 
where $\delta=$ coefficients of $X$, with $\delta=\beta(1-\alpha)$, and $w_{1}=$ error term.

In this step, we obtain the estimate of $\alpha$, i.e., the relative weight of the vulnerability variables.

(c) Using the t test, test the hypothesis that $\alpha=0$.

(d) If the hypothesis that $\alpha=0$ is not rejected, (i.e., the t statistic on the $\alpha$ is not statistically significant), we can accept (i.e., not reject) model B as the true model because $\hat{Y}^{A}$ included in model $\mathrm{D}$, which represents the influence of vulnerability variables not included in model $\mathrm{B}$, has no additional explanatory power beyond that contributed by model B. In other words, model B encompasses model $A$ in the sense that the latter model does not contain any additional information that will improve the performance of model B. By the same token, if the null hypothesis is rejected, model B cannot be the true model.

(e) Then, we reverse the order of estimation of models A and B. We now estimate model B first, use the estimated $Y$ values from this model as regressor in model $\mathrm{D}$, repeat step (d), and decide whether to accept model A over model B. More specifically, we estimate the following model E:

Model E:

$$
Y=Z k+\theta \hat{Y}^{B}+w_{2}
$$

where $\hat{Y}^{B}=$ the estimated $Y$ values from model B, $\theta=$ coefficients of $\hat{Y}^{B}$, with $\theta \equiv(I-\alpha)$, i.e., the relative weight of the strength variables, $k=$ coefficients of $Z$, with $k=\alpha^{*} \gamma$, and $w_{2}=$ error term.

In this step, we obtain the estimate of $(1-\alpha)$, i.e., the relative weight of the strength variables.

We now test the hypothesis that $\theta=0$. If this hypothesis is not rejected, we choose model A over B. If the hypothesis that $\theta=0$ is rejected, choose B over A, as the latter does not improve over the performance of $\mathrm{B}$.

When one does a pair of nonnested tests, there are four possible outcomes, since each of model A and B may or may not be rejected. Furthermore, although it is intuitively appealing, the $J$ test will not be able to provide a clear answer if it leads to the acceptance or rejection of both models. In case both models are rejected, neither model helps to explain the behavior of Y. Similarly, if both models are accepted, as Kmenta notes, the data are apparently not rich enough to discriminate between the two hypotheses [models]."2 Or, as Davidson and MacKinnon note, "when neither model is rejected, we must conclude that both models apparently fit the data about equally well and that neither

\footnotetext{
${ }^{2}$ Kmenta, Jan, 1986, Elements of Econometrics, Macmillan, 2d ed., New York, p. 597.
} 
provides evidence that the other is misspecified. Presumably, either the two models are very similar, or the data set is not very informative.".

3. Applying the $\mathbf{J}$ test to data for the Eleventh Review, we find that neither model A nor B can be rejected, ${ }^{4}$ and as noted by Davidson and MacKinnon, a possible interpretation is that the traditional data set does not capture all available information. An important missing element could be the political agreements at the time of the Bretton Woods conference, whose influence has survived through the equiproportional element in subsequent quota increases.

\footnotetext{
${ }^{3}$ Davidson, Russell and James G. MacKinnon, 1993, Estimation and Inference in Econometrics, Oxford University Press, New York and Oxford, p. 383.

${ }^{4}$ See Part B of this note.
} 


\section{B. Nested Models With Vulnerability and Strength Variables}

The model with the vulnerability variables includes two variables: the variability of current receipts, VC, defined as one standard deviation from a five-year monthly average over a 13-year period (1982-94), and population, POP, in 1994. The model with the strength variables includes four variables: GDP, Y, in 1994; the average monthly reserves with gold valued at market prices, RM, in 1994; the annual average of current receipts over a five-year. period (1990-94), C; and the four-year moving average of net private capital flows (1991-94), NNKFL.

The attached table summarizes the regression results of two nested models and a linear equation used to estimate the relative weights of strength and vulnerability variables. The first nested model is estimated using fitted values for the vulnerability model (Regression No. 53), while the second nested model is estimated using fitted values for the strength model (Regression No. 54). The coefficient estimates of the linear equation of actual quotas on both strength and vulnerability variables (Regression No. 55) are OLS estimates. The coefficients shown in this table are weighted coefficients, equal to the product of the estimated coefficients from the respective regressions times their corresponding weights $(\alpha)$ and $(1-\alpha)$. 
Table IV.1. Comparison of Coefficients from Nested Models and Ordinary Least Squares to Estimate the Relative Weights of Strength and Vulnerability Variables

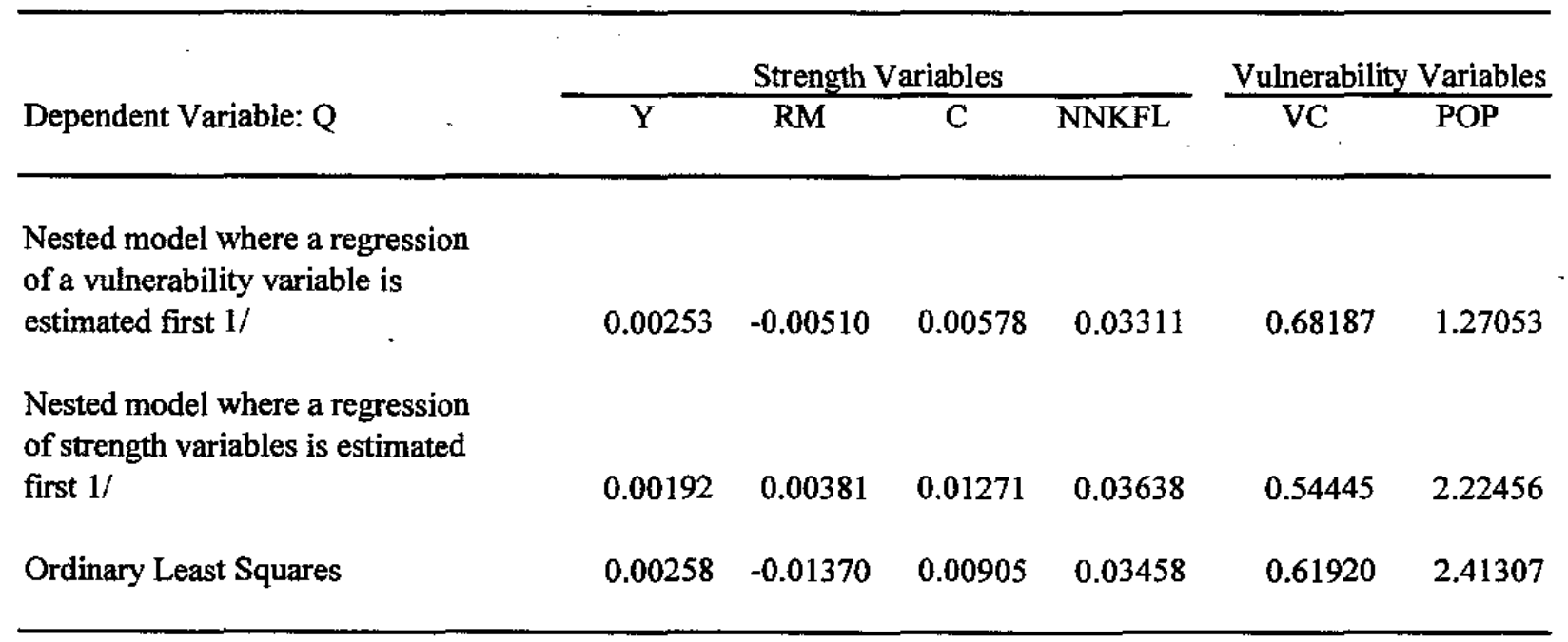

$\mathrm{Q}$ is the actual quota; $\mathrm{Y}$ is GDP in a recent year (1994); RM is average monthly reserves with gold valued at market prices in a recent year (1994); $\mathrm{C}$ is the annual average current receipts over a recent five-year period (1990-94); NNKFL is the four-year moving average of net private capital flows (1991-94).

$\mathrm{VC}$ is the variability of current receipts, defined as one standard deviation from a five-year moving average over a recent 13-year period (1982-1994); POP is population in 1994.

1/ Coefficients shown have been multiplied by the estimated relative weights, for the strength and vulnerability variables, i.e., they represent the "net" effect of the variable on the estimated quota. 


\section{Section V. Hypothetical Quota Calculations for Past Quota Reviews ACCORDING TO THE RELATIVE SIZE OF THE EQUIPROPORTIONAL AND SELECTIVE ELEMENTS}

This section presents a summary table on simulated quota shares from the Sixth through the Eleventh Quota Reviews had the distribution of quota increases been consistently either fully equiproportional, fully selective, or evenly divided between the equiproportional and selective elements. .

For this exercise, we use a constant sample of countries participating in the Sixth Review (121 members) plus China for the Seventh through the Eleventh Reviews, and take as given the size of the overall quota increases that were agreed. The simulation calculations are structured as follows: For the Seventh (initial) Review, in the fully equiproportional distribution scheme, the overall quota increase for the Seventh Review (50.9 percent) is applied to each member's actual (proposed) quotas under the Sixth Review to obtain the "fully equiproportional" quotas. In the fully selective distribution scheme, the overall percentage quota increase ( 50.9 percent) is first applied to the sum of the actual (proposed) quotas of the Sixth Review for the 122 members in the sample, and then distributed according to each member's calculated quota share in the total (Seventh Review) sample to obtain the "fully selective" quotas. The outcome of the evenly divided distribution scheme is generated as the average of the thus resulting "fully equiproportional" and "fully selective" quotas. For the Eighth through the Eleventh Reviews, the overall percentage increase in quotas for each review is applied in the same manner as described for the Seventh Review, except that calculations are based on quotas generated from the simulation of the previous review, instead of actual (proposed) quotas from the previous review.

The results indicate that if the distribution of past quota increases had been (1) fully equiproportional, the United States, the United Kingdom, India, and China would have ended up with higher quota shares under the Eleventh Review, while Japan, Germany, France, Italy, and Saudi Arabia would have ended up with lower quota shares; (2) fully selective, the reverse would have held true, except for the United States and the United Kingdom, which are marginally affected, and Saudi Arabia, which gains; and (3) evenly divided between the equiproportional and selective elements, the simulated quota shares under the Eleventh Review for the United States and the United Kingdom would be higher, while those for Saudi Arabia and Japan would be lower and for Germany would be unchanged, compared with the actual quota shares under the Eleventh Review. 
Table V.1. Summary of Simulated Quota Shares from Sixth Through Eleventh Reviews

(In percent of total)

\begin{tabular}{|c|c|c|c|c|c|c|c|c|c|c|c|c|}
\hline \multirow[b]{2}{*}{ Country } & \multirow{2}{*}{$\begin{array}{c}\text { Initial } \\
\text { (Actual) } \\
\text { Quota from } \\
\text { Sixth } \\
\text { Review }\end{array}$} & \multicolumn{3}{|c|}{$\begin{array}{l}\text { Simulated Quota } \\
\text { Eleventh Review }\end{array}$} & \multirow{2}{*}{$\begin{array}{l}\text { Actual } \\
\text { Quota } \\
\text { from } \\
\text { Eleventh } \\
\text { Review }\end{array}$} & \multirow{2}{*}{$\begin{array}{c}\text { Difference I } \\
\text { Equi- } \\
\text { proportional }\end{array}$} & \multirow{2}{*}{ 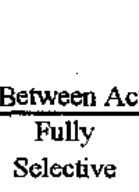 } & \multirow{2}{*}{$\begin{array}{l}\text { Eval and } \\
\text { Divided }\end{array}$} & \multirow{2}{*}{$\begin{array}{l}\text { Calculated } \\
\text { Quota } \\
\text { Shares fi } \\
\text { Eleventh } \\
\text { Review }\end{array}$} & \multicolumn{3}{|c|}{$\begin{array}{c}\text { Ratio of Simulated Quota Shares } \\
\text { to Eleventh Review } \\
\text { Calculated Quota Shares }\end{array}$} \\
\hline & & $\begin{array}{c}\text { Equi- } \\
\text { proportional }\end{array}$ & $\begin{array}{l}\text { Fully } \\
\text { Selective }\end{array}$ & $\begin{array}{c}\text { Evenly } \\
\text { Divided }\end{array}$ & & & & & & $\begin{array}{c}\text { Equi- } \\
\text { proportional }\end{array}$ & $\begin{array}{c}\text { Fully } \\
\text { Selective }\end{array}$ & $\begin{array}{l}\text { Evenly } \\
\text { Divided }\end{array}$ \\
\hline Algeria & 0.725 & 0.725 & 0.561 & 0.643 & 0.655 & -0.07 & 0.09 & 0.01 & 0.357 & 2.03 & 1.57 & 1.80 \\
\hline Argentina & 1.361 & 1.361 & 0.812 & 1.087 & 1.105 & -0.26 & 0.29 & 0.02 & 0.688 & 1.98 & 1.18 & 1.58 \\
\hline Australia & 2.010 & 2.010 & 1.446 & 1.728 & 1.689 & -0.32 & 0.24 & -0.04 & 1.294 & 1.55 & 1.12 & 1.34 \\
\hline Austria & 0.840 & 0.840 & 1.100 & 0.970 & 0.977 & 0.14 & -0.12 & 0.01 & 1.376 & 0.61 & 0.80 & 0.70 \\
\hline Bahamas, The & 0.084 & 0.084 & 0.052 & 0.068 & 0.068 & -0.02 & 0.02 & 0.00 & 0.038 & 2.23 & 1.38 & 1.80 \\
\hline Bahrain & 0.051 & 0.051 & 0.091 & 0.071 & 0.070 & 0.02 & -0.02 & 0.00 & 0.116 & 0.44 & 0.78 & 0.61 \\
\hline Bangladesh & 0.387 & 0.387 & 0.160 & 0.273 & 0.278 & -0.11 & 0.12 & 0.00 & 0.096 & 4.05 & 1.67 & 2.86 \\
\hline Barbados & 0.043 & 0.043 & 0.027 & 0.035 & 0.035 & -0.01 & 0.01 & 0.00 & 0.024 & 1.82 & 1.15 & 1.49 \\
\hline Belgium \& Luxembourg & 2.344 & 2.344 & 2.697 & 2.520 & 2.548 & 0.20 & -0.15 & 0.03 & 2.929 & 0.80 & 0.92 & 0.86 \\
\hline Benin & 0.041 & 0.041 & 0.023 & 0.032 & 0.032 & -0.01 & 0.01 & 0.00 & 0.014 & 2.81 & 1.56 & 2.19 \\
\hline Bolivia & 0.115 & 0.115 & 0.060 & 0.087 & 0.089 & -0.03 & 0.03 & 0.00 & 0.031 & 3.73 & 1.97 & 2.85 \\
\hline Botswana & 0.023 & 0.023 & 0.044 & 0.034 & 0.033 & 0.01 & -0.01 & 0.00 & 0.071 & 0.32 & 0.63 & 0.48 \\
\hline Brazil & 1.692 & 1.692 & 1.590 & 1.641 & 1.584 & -0.11 & -0.01 & -0.06 & 1.476 & 1.15 & 1.08 & 1.11 \\
\hline Burkina Faso & 0.041 & 0.041 & 0.020 & 0.031 & 0.031 & -0.01 & 0.01 & 0.00 & 0.013 & 3.21 & 1.61 & 2.41 \\
\hline Burundi & 0.059 & 0.059 & 0.018 & 0.038 & 0.040 & -0.02 & 0.02 & 0.00 & 0.006 & 9.71 & 3.04 & 6.38 \\
\hline Cameroon & 0.115 & 0.115 & 0.078 & 0.096 & 0.097 & -0.02 & 0.02 & 0.00 & 0.056 & 2.03 & 1.38 & 1.71 \\
\hline Canada & 3.453 & 3.453 & 3.387 & 3.420 & 3.323 & -0.13 & -0.06 & -0.10 & 3.520 & 0.98 & 0.96 & 0.97 \\
\hline Central African Republic & 0.041 & 0.041 & 0.015 & 0.028 & 0.029 & -0.01 & 0.01 & 0.00 & 0.007 & 5.80 & 2.17 & 3.98 \\
\hline Chad & 0.041 & 0.041 & 0.016 & 0.028 & 0.029 & -0.01 & 0.01 & 0.00 & 0.008 & 4.89 & 1.91 & 3.40 \\
\hline Chile & 0.552 & 0.552 & 0.342 & 0.447 & 0.447 & -0.11 & 0.11 & 0.00 & 0.276 & 2.00 & 1.24 & 1.62 \\
\hline China & 3.053 & 3.053 & 1.918 & 2.486 & 2.445 & -0.61 & 0.53 & -0.04 & 1.785 & 1.71 & 1.07 & 1.39 \\
\hline Colombia & 0.491 & 0.491 & 0.314 & 0.402 & 0.404 & -0.09 & 0.09 & 0.00 & 0.261 & 1.88 & 1.20 & 1.54 \\
\hline Congo, Dem. Republic of & 0.387 & 0.387 & 0.157 & 0.272 & 0.278 & -0.11 & 0.12 & 0.01 & 0.059 & 6.51 & 2.64 & 4.57 \\
\hline Congo, Republic of & 0.043 & 0.043 & 0.045 & 0.044 & 0.044 & 0.00 & 0.00 & 0.00 & 0.045 & 0.96 & 1.00 & 0.98 \\
\hline Costa Rica & 0.104 & 0.104 & 0.067 & 0.086 & 0.086 & -0.02 & 0.02 & 0.00 & 0.056 & 1.87 & 1.20 & 1.53 \\
\hline Cote d'Ivoire & 0.193 & 0.193 & 0.133 & 0.163 & 0.170 & -0.02 & 0.04 & 0.01 & 0.075 & 2.58 & 1.78 & 2.18 \\
\hline Сургus & 0.087 & 0.087 & 0.063 & 0.075 & 0.073 & -0.01 & 0.01 & 0.00 & 0.065 & 1.33 & 0.96 & 1.14 \\
\hline Denmark & 0.789 & 0.789 & 0.920 & 0.854 & 0.857 & 0.07 & -0.06 & 0.00 & 1.076 & 0.73 & 0.85 & 0.79 \\
\hline Dominican Republic & 0.140 & 0.340 & 0.089 & 0.114 & 0.114 & -0.03 & 0.03 & 0.00 & 0.073 & 1.92 & 1.22 & 1.57 \\
\hline Ecuador & 0.178 & 0.178 & 0.137 & 0.158 & 0.158 & -0.02 & 0.02 & 0.00 & 0.102 & 1.74 & 1.34 & 1.54 \\
\hline Egypt & 0.580 & 0.580 & 0.422 & 0.501 & 0.492 & -0.09 & 0.07 & -0.01 & 0.406 & 1.43 & 1.04 & 1.24 \\
\hline El Salvador & 0.109 & 0.109 & 0.064 & 0.087 & 0.089 & -0.02 & 0.03 & 0.00 & 0.038 & 2.88 & 1.68 & 2.28 \\
\hline Equatorial Guinea & 0.025 & 0.025 & 0.007 & 0.016 & 0.017 & -0.01 & 0.01 & 0.00 & 0.002 & 15.82 & 4.52 & 10.17 \\
\hline Ethiopia & 0.092 & 0.092 & 0.046 & 0.069 & 0.070 & -0.02 & 0.02 & 0.00 & 0.026 & 3.52 & 1.78 & 2.65 \\
\hline Fiji & 0.046 & 0.046 & 0.027 & 0.037 & 0.037 & -0.01 & 0.01 & 0.00 & 0.022 & 2.11 & 1.26 & 1.69 \\
\hline Finland & 0.667 & 0.667 & 0.659 & 0.663 & 0.659 & -0.01 & 0.00 & 0.00 & 0.684 & 0.98 & 0.96 & 0.97 \\
\hline France & 4.883 & 4.883 & 5.594 & 5.238 & 5.603 & 0.72 & 0.01 & 0.36 & 6.008 & 0.81 & 0.93 & 0.87 \\
\hline Gabon & 0.076 & 0.076 & 0.086 & 0.081 & 0.081 & 0.00 & -0.01 & 0.00 & 0.076 & 1.01 & 1.13 & 1.07 \\
\hline Gambia, The & 0.023 & 0.023 & 0.009 & 0.016 & 0.016 & -0.01 & 0.01 & 0.00 & 0.006 & 3.98 & 1.55 & 2.77 \\
\hline Germany & 5.486 & 5.486 & 8.069 & 6.778 & 6.787 & 1.30 & -1.28 & 0.01 & 9,698 & 0.57 & 0.83 & 0.70 \\
\hline Ghana & 0.270 & 0.270 & 0.100 & 0.185 & 0.193 & -0.08 & 0.09 & 0.01 & 0.031 & 8.79 & 3.27 & 6.03 \\
\hline Greece & 0.471 & 0.471 & 0.419 & 0.445 & 0.429 & -0.04 & 0.01 & -0.02 & 0.413 & 1.14 & 1.01 & 1.08 \\
\hline Grenada & 0.008 & 0.008 & 0.004 & 0.006 & 0.006 & 0.00 & 0.00 & 0.00 & 0.003 & 2.33 & 1.15 & 1.74 \\
\hline Guatemala & 0.130 & 0.130 & 0.084 & 0.107 & 0.110 & -0.02 & 0.03 & 0.00 & 0.051 & 2.53 & 1.64 & 2.08 \\
\hline Guinea & 0.076 & 0.076 & 0.033 & 0.055 & 0.056 & -0.02 & 0.02 & 0.00 & 0.021 & 3.69 & 1.58 & 2.64 \\
\hline
\end{tabular}


Table V.1. Summary of Simulated Quota Shares from Sixth Through Eleventh Reviews

(In percent of total)

\begin{tabular}{|c|c|c|c|c|c|c|c|c|c|c|c|c|}
\hline \multirow[b]{2}{*}{ Country } & \multirow{2}{*}{$\begin{array}{c}\text { Initial } \\
\text { (Actual) } \\
\text { Quota from } \\
\text { Sixth } \\
\text { Review }\end{array}$} & \multicolumn{3}{|c|}{$\begin{array}{l}\text { Simulated Quota- } \\
\text { Eleventh Review }\end{array}$} & \multirow{2}{*}{$\begin{array}{c}\text { Actual } \\
\text { Quota } \\
\text { from } \\
\text { Eleventh } \\
\text { Review }\end{array}$} & \multirow{2}{*}{$\begin{array}{c}\text { Difference } \\
\begin{array}{c}\text { Equi- } \\
\text { proportional }\end{array}\end{array}$} & \multirow{2}{*}{ 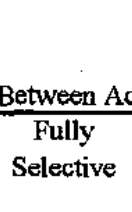 } & \multirow{2}{*}{$\begin{array}{l}\text { ctual and } \\
\text { Evenly } \\
\text { Divided }\end{array}$} & \multirow{2}{*}{$\begin{array}{l}\text { Calculated } \\
\text { Quota } \\
\text { Shares fr } \\
\text { Eleventh } \\
\text { Review }\end{array}$} & \multicolumn{3}{|c|}{$\begin{array}{c}\text { Ratio of Simulated Quota Shares } \\
\text { to Eleventh Review } \\
\text { Calculated Quota Shares }\end{array}$} \\
\hline & & $\begin{array}{c}\text { Equi- } \\
\text { proportional }\end{array}$ & $\begin{array}{c}\text { Fully } \\
\text { Selective }\end{array}$ & $\begin{array}{l}\text { Evenly } \\
\text { Divided }\end{array}$ & & & & & & $\begin{array}{l}\text { Equi- } \\
\text { proportional }\end{array}$ & $\begin{array}{c}\text { Fully } \\
\text { Selective }\end{array}$ & $\begin{array}{l}\text { Evenly } \\
\text { Divided }\end{array}$ \\
\hline Guyana & 0.064 & 0.064 & 0.029 & 0.046 & 0.047 & -0.02 & 0.02 & 0.00 & 0.012 & 5.27 & 2.43 & 3.85 \\
\hline Haiti & 0.059 & 0.059 & 0.023 & 0.041 & 0.043 & -0.02 & 0.02 & 0.00 & 0.008 & 6.93 & 2.77 & 4.85 \\
\hline Honduras & 0.087 & 0.087 & 0.045 & 0.066 & 0.068 & -0.02 & 0.02 & 0.00 & 0.027 & 3.16 & 1.65 & 2.40 \\
\hline Iceland & 0.074 & 0.074 & 0.049 & 0.062 & 0.061 & -0.01 & 0.01 & 0.00 & 0.039 & 1.89 & 1.26 & 1.58 \\
\hline India & 2.913 & 2.913 & 1.333 & 2.123 & 2.169 & -0.74 & 0.84 & 0.05 & 0.820 & 3.55 & 1.62 & 2.59 \\
\hline Indonesia & 1.221 & 1.221 & 0.982 & 1.102 & 1.085 & -0.14 & 0.10 & -0.02 & 0.852 & 1.43 & 1.15 & 1.29 \\
\hline Iran & 1.679 & 1.679 & 1.393 & 1.536 & 0.781 & -0.90 & -0.61 & -0.76 & 0.611 & 2.75 & 2.28 & 2.51 \\
\hline Iraq & 0.359 & 0.359 & 0.740 & 0.549 & 0.620 & 0.26 & -0.12 & 0.07 & 0.357 & 1.00 & 2.07 & 1.54 \\
\hline Ireland & 0.394 & 0.394 & 0.480 & 0.437 & 0.437 & 0.04 & -0.04 & 0.00 & 0.655 & 0.60 & 0.73 & 0.67 \\
\hline Israel & 0.522 & 0.522 & 0.461 & 0.492 & 0.484 & -0.04 & 0.02 & -0.01 & 0.415 & 1.26 & 1.11 & 1.19 \\
\hline Italy & 3.155 & 3.155 & 3.903 & 3.529 & 3.681 & 0.53 & -0.22 & 0.15 & 4.501 & 0.70 & 0.87 & 0.78 \\
\hline Jamaica & 0.188 & 0.188 & 0.095 & 0.142 & 0.143 & -0.05 & 0.05 & 0.00 & 0.055 & 3.40 & 1.72 & 2.56 \\
\hline Japan & 4.221 & 4.221 & 8.051 & 6.136 & 6.946 & 2.72 & -1.11 & 0.81 & 10.987 & 0.38 & 0.73 & 0.56 \\
\hline Jordan & 0.076 & 0.076 & 0.100 & 0.088 & 0.089 & 0.01 & $-0,01$ & 0.00 & 0.086 & 0.89 & 1.16 & 1.03 \\
\hline Kenya & 0.176 & 0.176 & 0.098 & 0.137 & 0.142 & -0.03 & 0.04 & 0.00 & 0.054 & 3.25 & 1.81 & 2.53 \\
\hline Korea & 0.407 & 0.407 & 1.101 & 0.754 & 0.852 & 0.45 & -0.25 & 0.10 & 1.786 & 0.23 & 0.62 & 0.42 \\
\hline Kuwait & 0.598 & 0.598 & 0.773 & 0.685 & 0.721 & 0.12 & -0.05 & 0.04 & 0.559 & 1.07 & 1.38 & 1.23 \\
\hline Lao, People's Dem. Repub & 0.041 & 0.041 & 0.013 & 0.027 & 0.028 & -0.01 & 0.01 & 0.00 & 0.007 & 5.93 & 1.89 & 3.91 \\
\hline Lebanon & 0.031 & 0.031 & 0.162 & 0.096 & 0.106 & 0.08 & -0.06 & 0.01 & 0.086 & 0.35 & 1.89 & 1.12 \\
\hline Lesotho & 0.018 & 0.018 & 0.018 & 0.018 & 0.018 & 0.00 & 0.00 & 0.00 & 0.019 & 0.96 & 0.98 & 0.97 \\
\hline Liberia & 0.094 & 0.094 & 0.034 & 0.064 & 0.067 & -0.03 & 0.03 & 0.00 & 0.007 & 14.12 & 5.14 & 9.63 \\
\hline Libya & 0.471 & 0.471 & 0.620 & 0.545 & 0.586 & 0.12 & -0.03 & 0.04 & 0.340 & 1.39 & 1.82 & 1.60 \\
\hline Madagascar & 0.087 & 0.087 & 0.036 & 0.061 & 0.064 & -0.02 & 0.03 & 0.00 & 0.015 & 5.81 & 2.42 & 4.12 \\
\hline Malawi & 0.048 & 0.048 & 0.023 & 0.035 & 0.036 & -0.01 & 0.01 & 0.00 & 0.015 & 3.30 & 1.54 & 2.42 \\
\hline Malaysia & 0.644 & 0.644 & 0.766 & 0.705 & 0.776 & 0.13 & 0.01 & 0.07 & 1.107 & 0.58 & 0.69 & 0.64 \\
\hline Mali & 0.069 & 0.069 & 0.026 & 0.047 & 0.049 & -0.02 & 0.02 & 0.00 & 0.014 & 4.99 & 1.87 & 3.43 \\
\hline Malta & 0.051 & 0.051 & 0.054 & 0.053 & 0.053 & 0.00 & 0.00 & 0.00 & 0.062 & 0.82 & 0.87 & 0.84 \\
\hline Mauritania & 0.043 & 0.043 & 0.022 & 0.033 & 0.034 & -0.01 & 0.01 & 0.00 & 0.010 & 4.14 & 2.09 & 3.12 \\
\hline Mauritius & 0.069 & 0.069 & 0.040 & 0.054 & 0.053 & -0.02 & 0.01 & 0.00 & 0.039 & 1.75 & 1.02 & 1.38 \\
\hline Mexico & 1.361 & 1.361 & 1.310 & 1.336 & 1.349 & -0.01 & 0.04 & 0.01 & 1.430 & 0.95 & 0.92 & 0.93 \\
\hline Morocco & 0.382 & 0.382 & 0.234 & 0.308 & 0.307 & -0.07 & 0.07 & 0.00 & 0.181 & 2.10 & 1.29 & 1.70 \\
\hline Myanmar & 0.186 & 0.186 & 0.096 & 0.141 & 0.135 & -0.05 & 0.04 & -0.01 & 0.124 & 1.50 & 0.78 & 1.14 \\
\hline Nepal & 0.048 & 0.048 & 0.026 & 0.037 & 0.037 & -0.01 & 0.01 & 0.00 & 0.020 & 2.44 & 1.30 & 1.87 \\
\hline Netherlands & 2.412 & 2.412 & 2.937 & 2.674 & 2.693 & 0.28 & -0.24 & 0.02 & 3.048 & 0.79 & 0.96 & 0.88 \\
\hline New Zealand & 0.590 & 0.590 & 0.352 & 0.471 & 0.467 & -0.12 & 0.11 & 0.00 & 0.282 & 2.09 & 1.25 & 1.67 \\
\hline Nicaragua & 0.087 & 0.087 & 0.044 & 0.065 & 0.068 & -0.02 & 0.02 & 0.00 & 0.017 & 5.15 & 2.63 & 3.89 \\
\hline Niger & 0.041 & 0.041 & 0.025 & 0.033 & 0.034 & -0.01 & 0.01 & 0.00 & 0.013 & 3.06 & 1.85 & 2.45 \\
\hline Nigeria & 0.916 & 0.916 & 0.856 & 0.886 & 0.915 & 0.00 & 0.06 & 0.03 & 0.443 & 2.07 & 1.93 & 2.00 \\
\hline Norway & 0.751 & 0.751 & 0.969 & 0.860 & 0.872 & 0.12 & -0.10 & 0.01 & 1.028 & 0.73 & 0.94 & 0.84 \\
\hline Oman & 0.051 & 0.051 & 0.151 & 0.101 & 0.101 & 0.05 & -0.05 & 0.00 & 0.165 & 0.31 & 0.92 & 0.61 \\
\hline
\end{tabular}


Table V.1. Summary of Simulated Quota Shares from Sirth Through Eleventh Reviews

(In percent of total)

\begin{tabular}{|c|c|c|c|c|c|c|c|c|c|c|c|c|}
\hline \multirow[b]{2}{*}{ Country } & \multirow{2}{*}{$\begin{array}{c}\text { Initial } \\
\text { (Actual) } \\
\text { Quota from } \\
\text { Sixth } \\
\text { Review }\end{array}$} & \multicolumn{3}{|c|}{$\begin{array}{l}\text { Simulated Quota } \\
\text { Eleventh Review }\end{array}$} & \multirow{2}{*}{$\begin{array}{l}\text { Actual } \\
\text { Quota } \\
\text { from } \\
\text { Eleventh } \\
\text { Review }\end{array}$} & \multirow{2}{*}{ 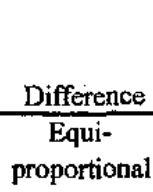 } & \multirow{2}{*}{ 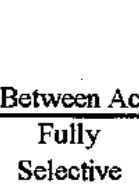 } & \multirow{2}{*}{$\begin{array}{l}\text { ctual and } \\
\text { Evenly } \\
\text { Divided }\end{array}$} & \multirow{2}{*}{$\begin{array}{l}\text { Calculated } \\
\text { Quota } \\
\text { Shares fr } \\
\text { Eleventh } \\
\text { Review }\end{array}$} & \multicolumn{3}{|c|}{$\begin{array}{c}\text { Ratio of Simulated Quota Shares } \\
\text { to Eleventh Review } \\
\text { Calculated Quota Shares }\end{array}$} \\
\hline & & $\begin{array}{c}\text { Equi- } \\
\text { proportional }\end{array}$ & $\begin{array}{c}\text { Fully } \\
\text { Selective }\end{array}$ & $\begin{array}{l}\text { Evenly } \\
\text { Divided }\end{array}$ & & & & & & $\begin{array}{c}\text { Equi- } \\
\text { proportional }\end{array}$ & $\begin{array}{l}\text { Fully } \\
\text { Selective }\end{array}$ & $\begin{array}{l}\text { Evenly } \\
\text { Divided }\end{array}$ \\
\hline Pakistan & 0.725 & 0.725 & 0.337 & 0.531 & 0.539 & -0.19 & 0.20 & 0.01 & 0.223 & 3.25 & 1.51 & 2.38 \\
\hline Panama & 0.115 & 0.115 & 0.095 & 0.105 & 0.108 & -0.01 & 0.01 & 0.00 & 0.073 & 1.56 & 1.30 & 1.43 \\
\hline Paraguay & 0.059 & 0.059 & 0.045 & 0.052 & 0.052 & -0.01 & 0.01 & 0.00 & 0.039 & 1.50 & 1.17 & 1.34 \\
\hline Peru & 0.417 & 0.417 & 0.242 & 0.330 & 0.333 & -0.08 & 0.09 & 0.00 & 0.169 & 2.46 & 1.43 & 1.95 \\
\hline Philippines & 0.534 & 0.534 & 0.402 & 0.468 & 0.459 & -0.08 & 0.06 & -0.01 & 0.366 & 1.46 & 1.10 & 1.28 \\
\hline Portugal & 0.438 & 0.438 & 0.469 & 0.453 & 0.453 & 0.01 & -0.02 & 0.00 & 0.601 & 0.73 & 0.78 & 0.75 \\
\hline Qatar & 0.102 & 0.102 & 0.161 & 0.131 & 0.138 & 0.04 & -0.02 & 0.01 & 0.101 & 1.01 & 1.60 & 1.31 \\
\hline Romania & 0.623 & 0.623 & 0.426 & 0.525 & 0.537 & -0.09 & 0.11 & 0.01 & 0.246 & 2.54 & 1.74 & 2.14 \\
\hline Rwanda & 0.059 & 0.059 & 0.021 & 0.040 & 0.042 & -0.02 & 0.02 & 0.00 & 0.006 & 9.44 & 3.35 & 6.40 \\
\hline Samoa & 0.008 & 0.008 & 0.004 & 0.006 & 0.006 & 0.00 & 0.00 & 0.00 & 0.002 & 3.32 & 1.54 & 2.43 \\
\hline Saudi Arabia & 1.527 & 1.527 & 2.996 & 2.261 & 3.645 & 2.12 & 0.65 & 1.38 & 1.413 & 1.08 & 2.12 & 1.60 \\
\hline Senegal & 0.107 & 0.107 & 0.058 & 0.082 & 0.084 & -0.02 & 0.03 & 0.00 & 0.031 & 3.41 & 1.85 & 2.63 \\
\hline Sierra Leone & 0.079 & 0.079 & 0.025 & 0.052 & 0.054 & -0.02 & 0.03 & 0.00 & 0.005 & 14.36 & 4.50 & 9.43 \\
\hline Singapore & 0.280 & 0.280 & 1.065 & 0.672 & 0.450 & 0.17 & -0.61 & -0.22 & 1.646 & 0.17 & 0.65 & 0.41 \\
\hline Somalia & 0.059 & 0.059 & 0.022 & 0.040 & 0.043 & .0 .02 & 0.02 & 0.00 & 0.003 & 17.13 & 6.34 & 11.73 \\
\hline South Africa & 1.079 & 1.079 & 0.839 & 0.959 & 0.975 & -0.10 & 0.14 & 0.02 & 0.480 & 2.25 & 1.75 & 2.00 \\
\hline Spain & 1.417 & 1.417 & 1.754 & 1.585 & 1.591 & 0.17 & -0.16 & 0.01 & 2.239 & 0.63 & 0.78 & 0.71 \\
\hline Sri Lanka & 0.303 & 0.303 & 0.122 & 0.213 & 0.216 & -0.09 & 0.09 & 0.00 & 0.084 & 3.60 & 1.45 & 2.52 \\
\hline Sudan & 0.224 & 0.224 & 0.094 & 0.159 & 0.164 & -0.06 & 0.07 & 0.01 & 0.039 & 5.71 & 2.39 & 4.05 \\
\hline Swaziland & 0.031 & 0.031 & 0.024 & 0.027 & 0.026 & 0.00 & 0.00 & 0.00 & 0.021 & 1.42 & 1.11 & 1.27 \\
\hline Sweden & 1.145 & 1.145 & 1.356 & 1.250 & 1.250 & 0.10 & -0.11 & 0.00 & 1.359 & 0.84 & 1.00 & 0.92 \\
\hline Syrian Arab Republic & 0.160 & 0.160 & 0.153 & 0.157 & 0.153 & -0.01 & 0.00 & 0.00 & 0.143 & 1.12 & 1.07 & 1.10 \\
\hline Tanzania & 0.140 & 0.140 & 0.061 & 0.101 & 0.104 & -0.04 & 0.04 & 0.00 & 0.028 & 4.97 & 2.18 & 3.57 \\
\hline Thailand & 0.461 & 0.461 & 0.567 & 0.514 & 0.564 & 0.10 & 0.00 & 0.05 & 0.928 & 0.50 & 0.61 & 0.55 \\
\hline Togo & 0.048 & 0.048 & 0.026 & 0.037 & 0.038 & -0.01 & 0.01 & 0.00 & 0.009 & 5.17 & 2.73 & 3.95 \\
\hline Trinidad and Tobago & 0.209 & 0.209 & 0.149 & 0.179 & 0.175 & -0.03 & 0.03 & 0.00 & 0.063 & 3.30 & 2.36 & 2.83 \\
\hline Tunisia & 0.160 & 0.160 & 0.143 & 0.152 & 0.149 & -0.01 & 0.01 & 0.00 & 0.123 & 1.31 & 1.16 & 1.24 \\
\hline Turkey & 0.509 & 0.509 & 0.510 & 0.509 & 0.503 & -0.01 & -0.01 & -0.01 & 0.574 & 0.89 & 0.89 & 0.89 \\
\hline Uganda & 0.127 & 0.127 & 0.050 & 0.089 & 0.094 & -0.03 & 0.04 & 0.01 & 0.017 & 7.45 & 2.96 & 5.20 \\
\hline United Arab Emirates & 0.305 & 0.305 & 0.568 & 0.437 & 0.319 & 0.01 & -0.25 & -0.12 & 0.430 & 0.71 & 1.32 & 1.02 \\
\hline United Kingdom & 7.443 & 7.443 & 5.784 & 6.613 & 5.603 & -1.84 & -0.18 & -1.01 & 5.375 & 1.38 & 1.08 & 1.23 \\
\hline United States & 21.387 & 21.387 & 19.453 & 20.420 & 19.382 & -2.00 & -0.07 & -1.04 & 18.575 & 1.15 & 1.05 & 1.10 \\
\hline Unuguay & 0.214 & 0.214 & 0.098 & 0.156 & 0.160 & -0.05 & 0.06 & 0.00 & 0.060 & 3.58 & 1.65 & 2.61 \\
\hline Venezuela & 1.679 & 1.679 & 1.046 & 1.363 & 1.387 & -0.29 & 0.34 & 0.02 & 0.563 & 2.98 & 1.86 & 2.42 \\
\hline Vietnam & 0.229 & 0.229 & 0.111 & 0.170 & 0.172 & -0.06 & 0.06 & 0.00 & 0.068 & 3.36 & 1.62 & 2.49 \\
\hline Yemen, Republic of & 0.137 & 0.137 & 0.109 & 0.123 & 0.127 & -0.01 & 0.02 & 0.00 & 0.083 & 1.65 & 1.31 & 1.48 \\
\hline Zambia & 0.359 & 0.359 & 0.140 & 0.250 & 0.255 & -0.10 & 0.11 & 0.01 & 0.036 & 9.92 & 3.88 & 6.90 \\
\hline Total & 100.0 & 100.0 & 100.0 & 100.0 & 100.0 & 0.0 & 0.0 & 0.0 & 100.0 & & & \\
\hline
\end{tabular}

